NISTIR 7822

\title{
SteelVis User's Guide (CIS/2 to VRML and IFC Translator)
}

Robert R. Lipman

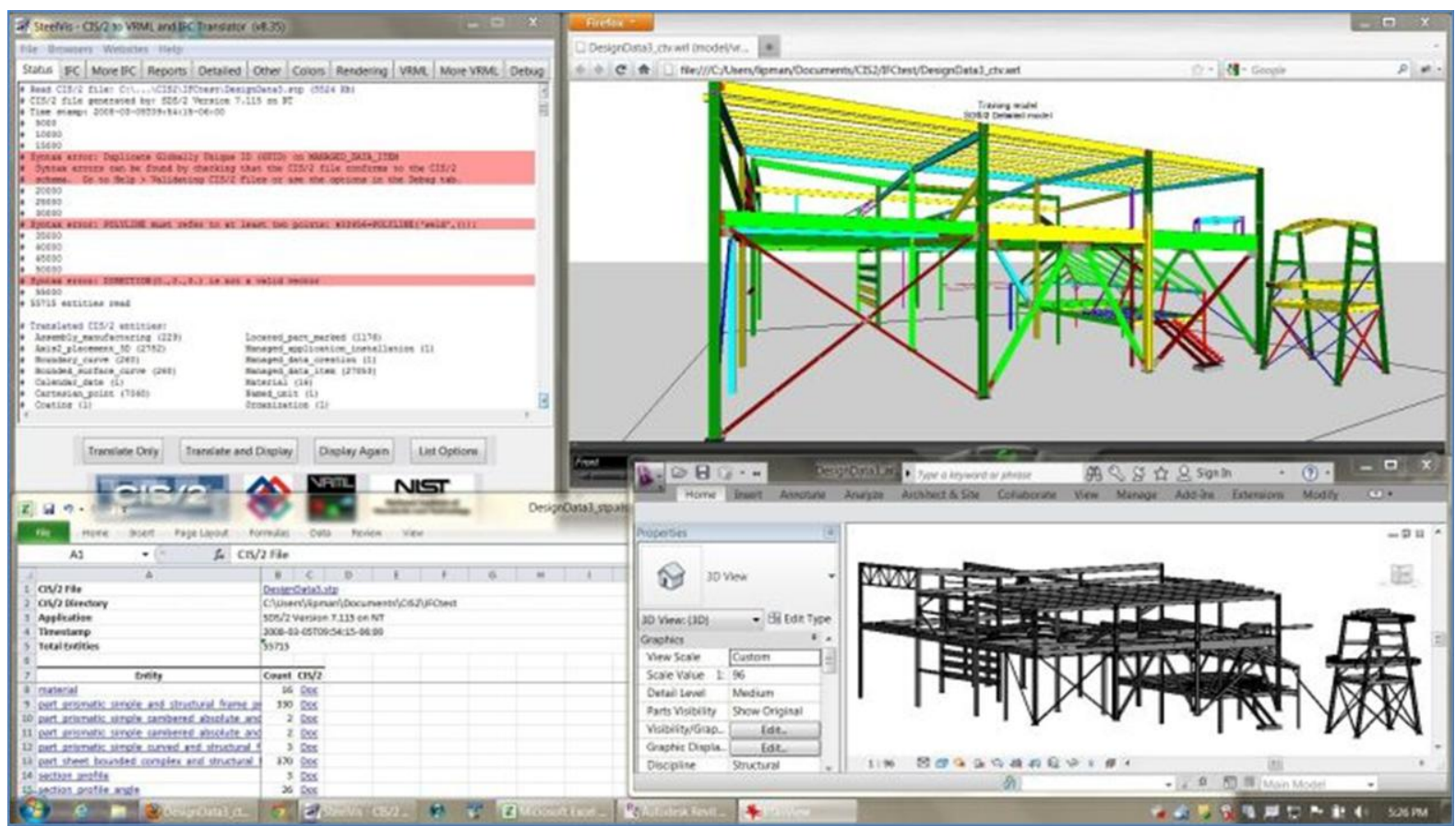

NIST

National Institute of Standards and Technology U.S. Department of Commerce 



\title{
SteelVis User's Guide (CIS/2 to VRML and IFC Translator)
}

\author{
Robert R. Lipman \\ Building Environment Division \\ Engineering Laboratory
}

September 2011

(Updated January 2012)

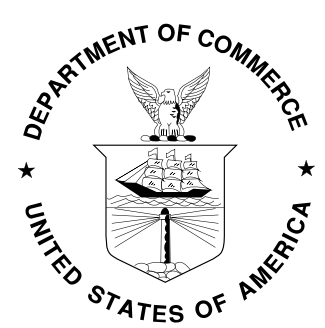

U.S. DEPARTMENT OF COMMERCE Rebecca Blank, Acting Secretary NATIONAL INSTITUTE OF STANDARDS AND TECHNOLOGY 



\section{PREFACE}

This guide describes how to use SteelVis and its associated programs. SteelVis has two main functions: (1) to translate a CIS/2 (CIMsteel Integration Standards) file into an interactive 3D model in the form of a VRML (Virtual Reality Modeling Language) file that can be displayed in a web browser VRML plugin or VRML viewer and (2) to translate a CIS/2 file into an IFC (Industry Foundation Classes) file that can be imported into many BIM (Building Information Modeling) applications. SteelVis also includes the CIS/2 File Analyzer that converts a CIS/ 2 file into a spreadsheet that can be used for inspecting and debugging the attributes, values, and relationships in the CIS/2 file.

SteelVis used to be known only as the CIS/2 to VRML and IFC Translator.

More information about SteelVis, CIS/2, and IFC can be found at http://cic.nist.gov/vrml/cis2.html.

\section{DISCLAIMERS}

Any mention of commercial products in SteelVis and this user's guide is for information purposes only; it does not imply recommendation or endorsement by NIST. For any of the web links in SteelVis and this user's guide, NIST does not necessarily endorse the views expressed, or concur with the facts presented on those web sites.

This software was developed at the National Institute of Standards and Technology by employees of the Federal Government in the course of their official duties. Pursuant to Title 17 Section 105 of the United States Code this software is not subject to copyright protection and is in the public domain. This software is an experimental system. NIST assumes no responsibility whatsoever for its use by other parties, and makes no guarantees, expressed or implied, about its quality, reliability, or any other characteristic.

Vendors of various software applications with CIS/2 export capabilities have supplied the sample CIS/2 files. The sample CIS/2 files do not necessarily reflect the current CIS/2 export capabilities of the software packages represented nor are all software packages that have implemented CIS $/ 2$ represented. NIST assumes no responsibility whatsoever for their use by other parties, and makes no guarantees, expressed or implied, about their quality, reliability, or any other characteristic.

This software can be redistributed and/or modified freely provided that any derivative works bear some notice that they are derived from it, and any modified versions bear some notice that they have been modified. We would appreciate acknowledgement if the software is used.

\section{ACKNOWLEDGEMENTS}

Most of the development of SteelVis was carried out between 2003 and 2006 as part of the project "Product Data Standards for Steel Construction" in the Computer Integrated Building Processes Group of the Building and Fire Research Laboratory at NIST. The author thanks Dr. Kent Reed, former leader of the group, for his support during the development of SteelVis. The author also acknowledges the many software vendors and users who have provided invaluable feedback, suggestions, and bug reports about SteelVis and supplied sample CIS/2 files that were used to test and improve the capabilities of SteelVis.

Cover image: Screenshot of SteelVis and associated types of output. Upper left - SteelVis user interface; upper right - VRML model; bottom right - IFC file displayed in BIM software; bottom left CIS/2 converted to a spreadsheet. 


\section{TABLE OF CONTENTS}

1 INTRODUCTION ................................................................................................................................ 1

2 GETTING STARTED .....................................................................................................................

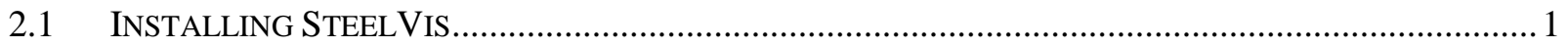

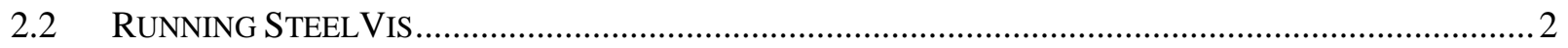

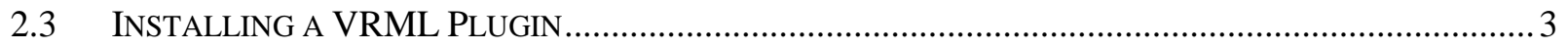

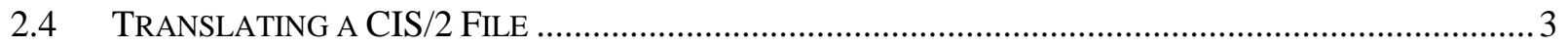

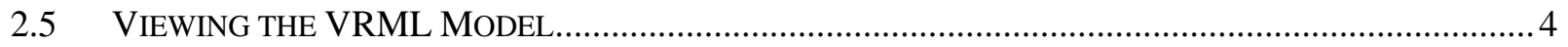

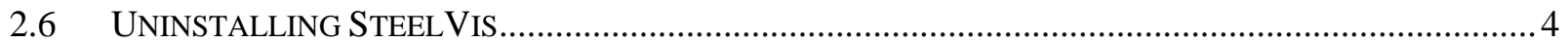

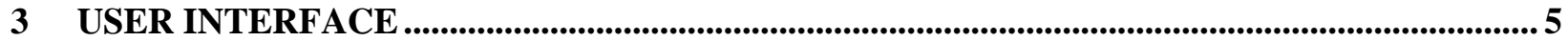

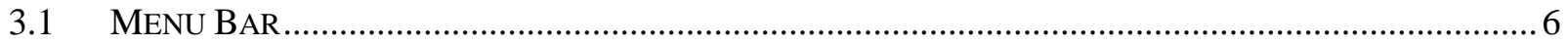

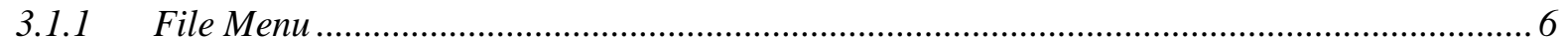

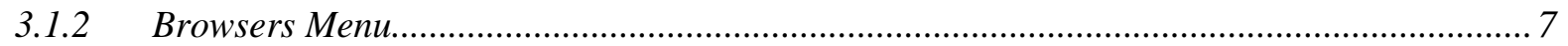

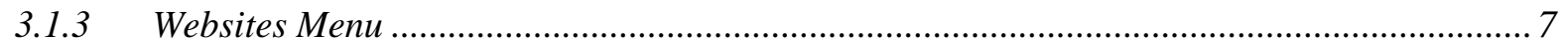

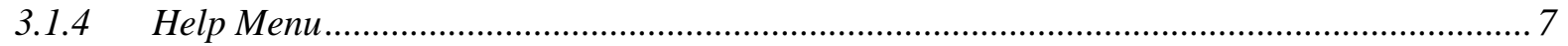

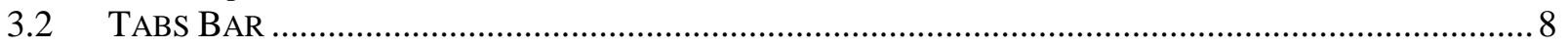

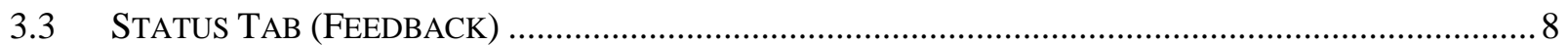

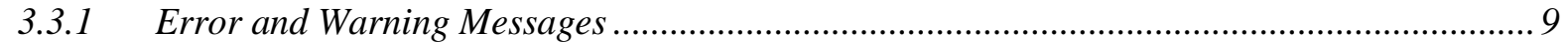

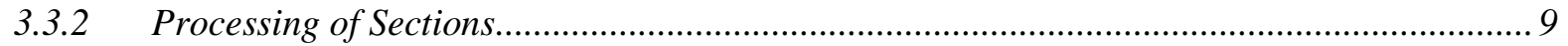

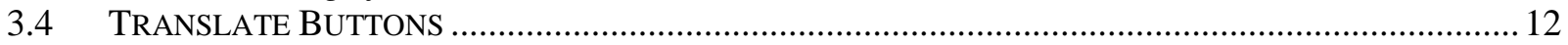

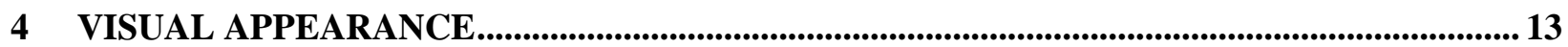

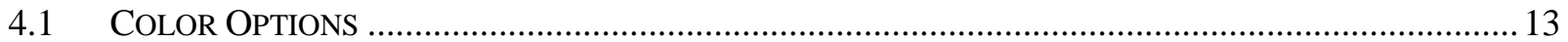

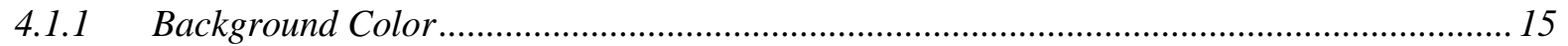

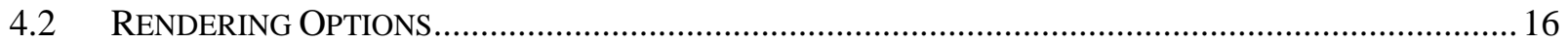

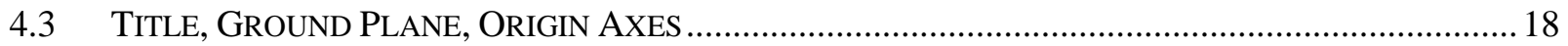

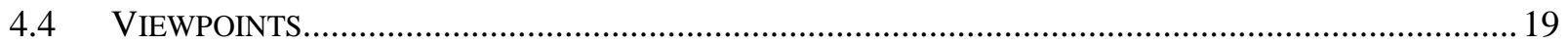

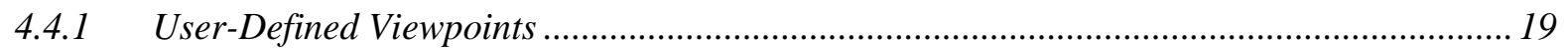

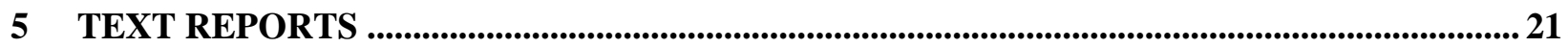

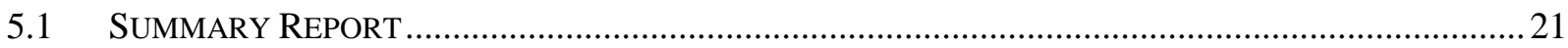

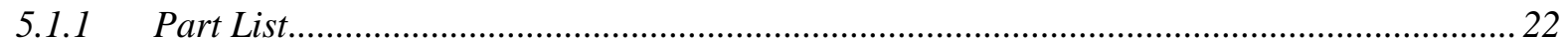

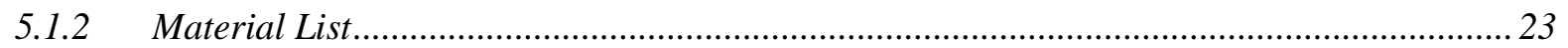

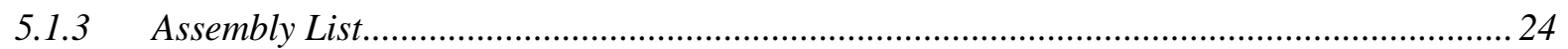

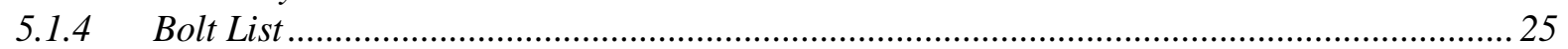

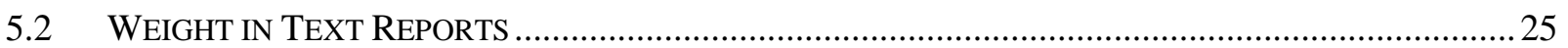

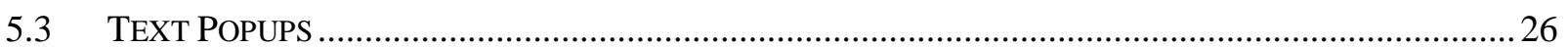

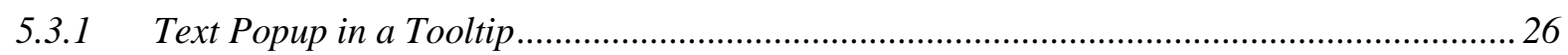

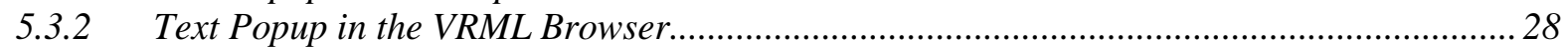

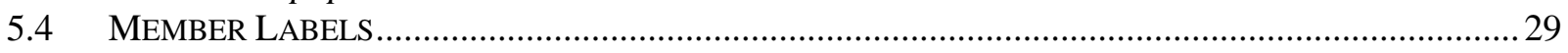

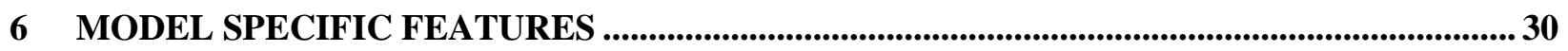

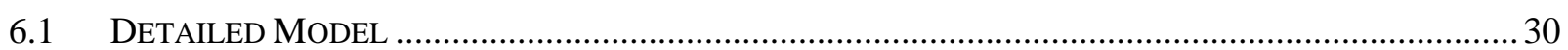

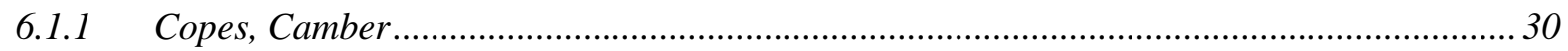

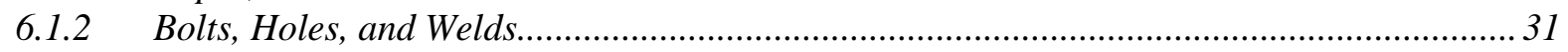

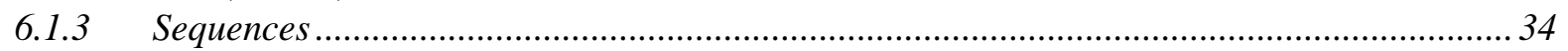

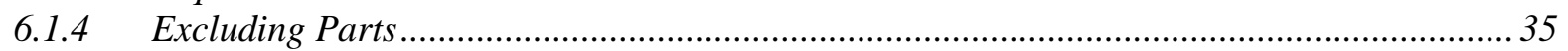

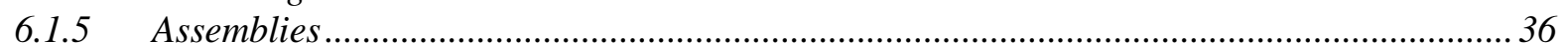




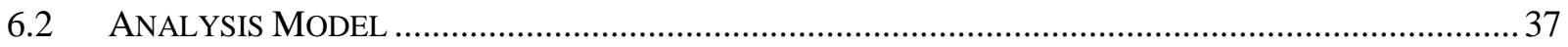

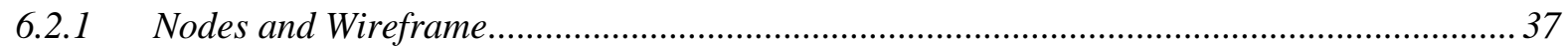

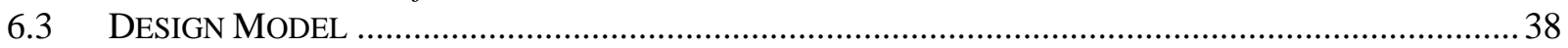

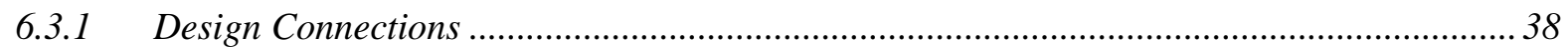

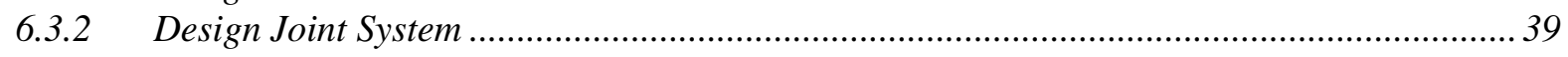

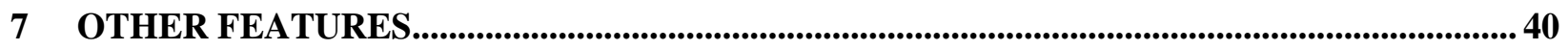

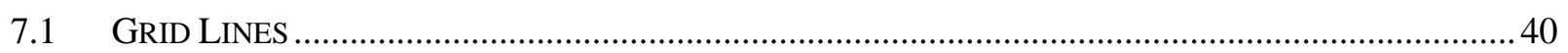

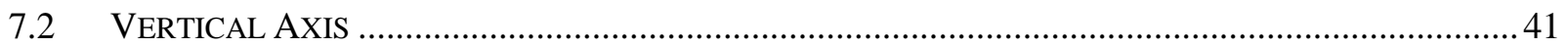

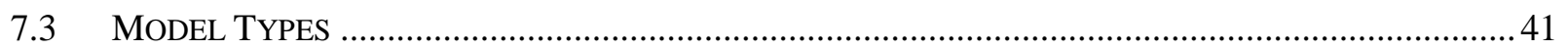

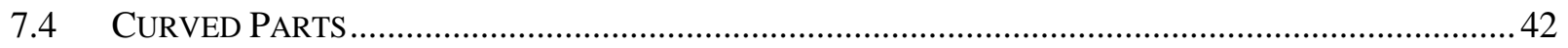

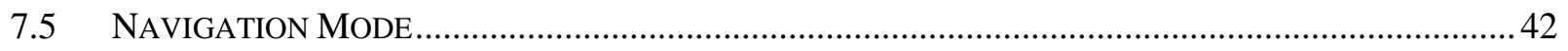

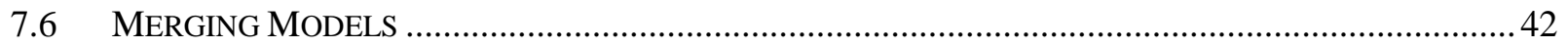

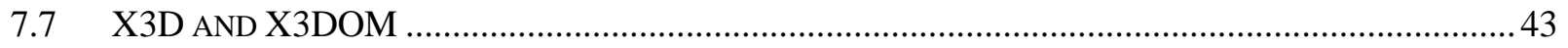

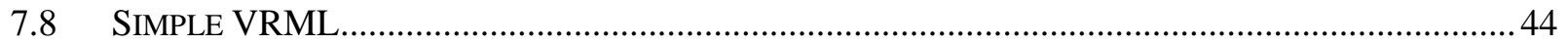

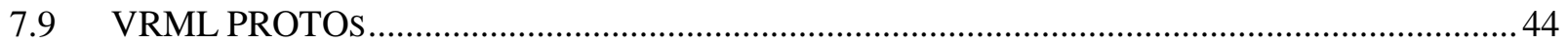

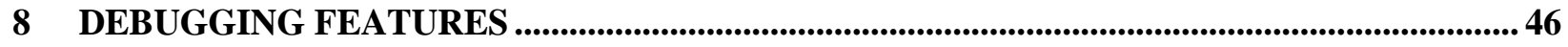

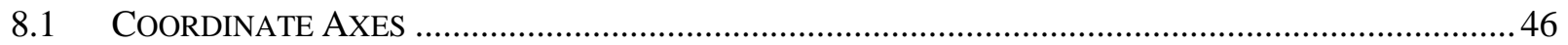

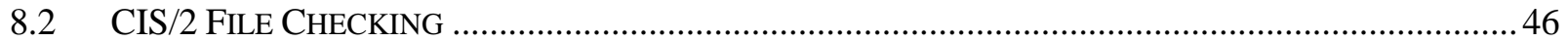

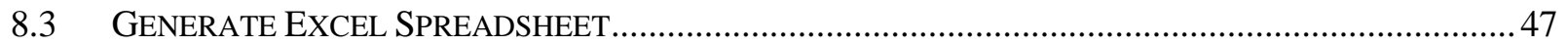

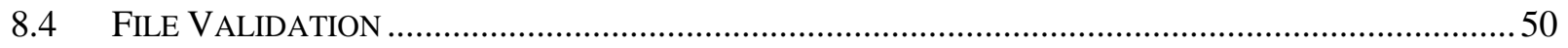

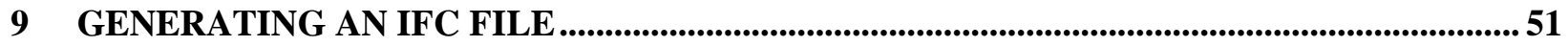

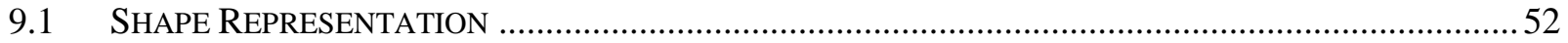

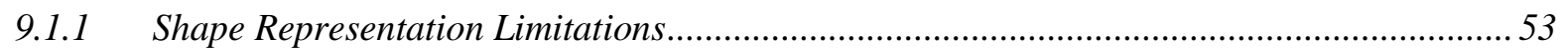

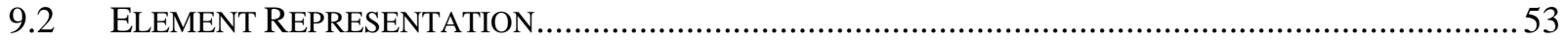

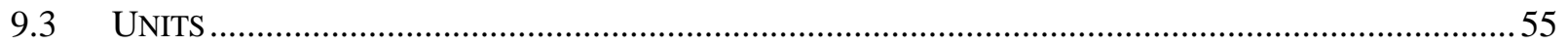

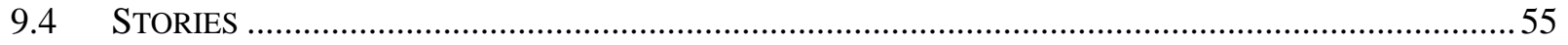

9.5 DISPLAYING THE IFC FILE

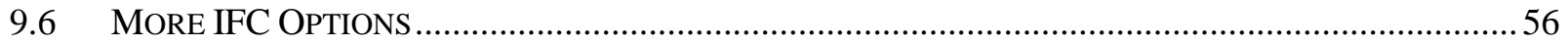

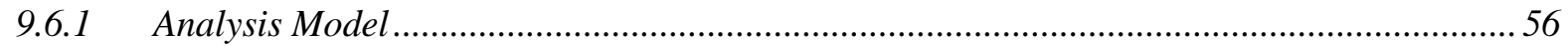

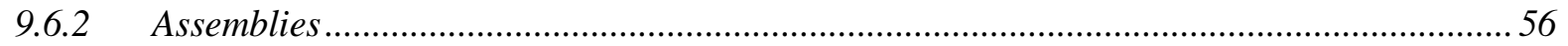

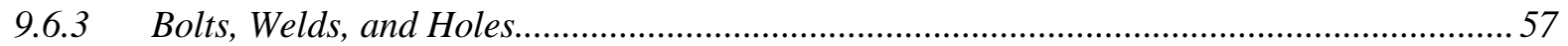

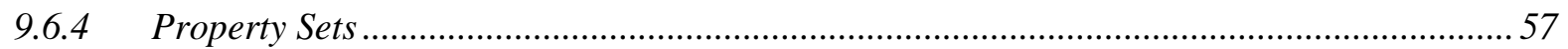

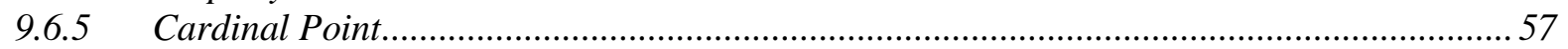

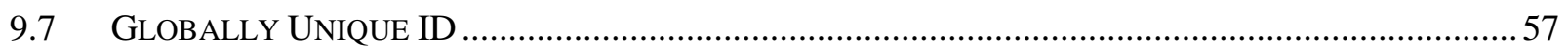

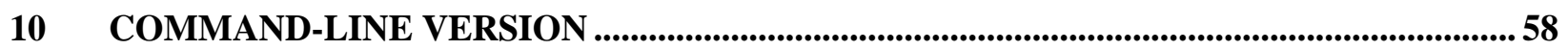

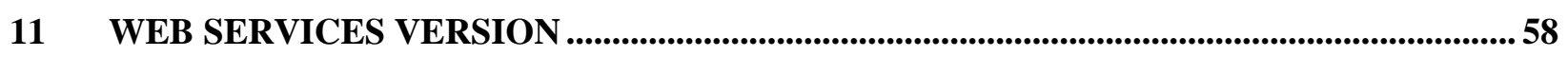

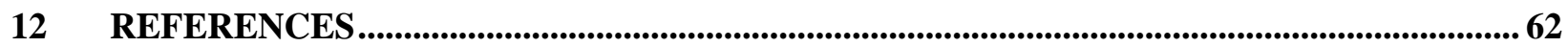




\section{LIST OF FIGURES}

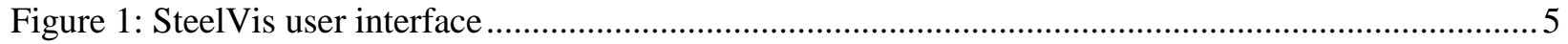

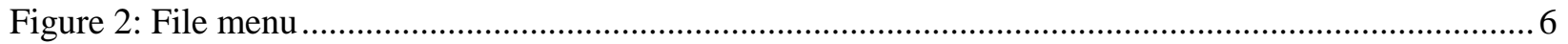

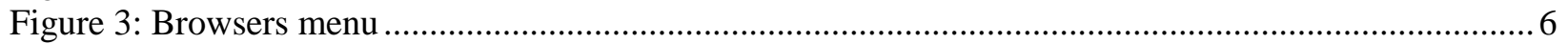

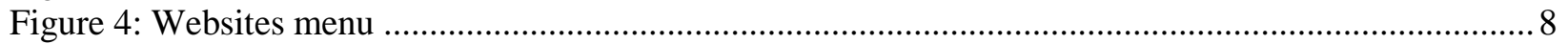

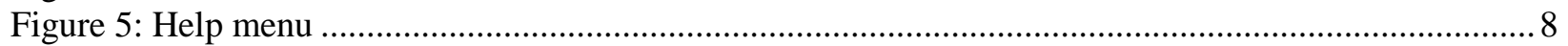

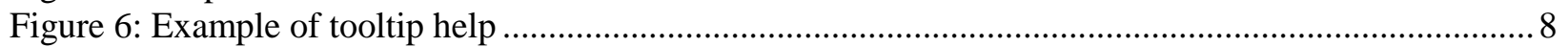

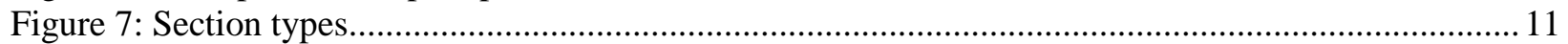

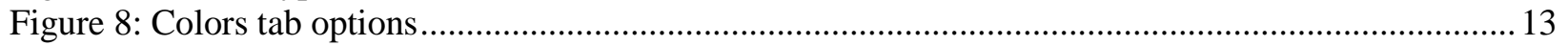

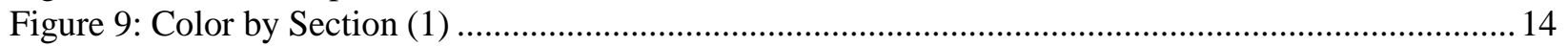

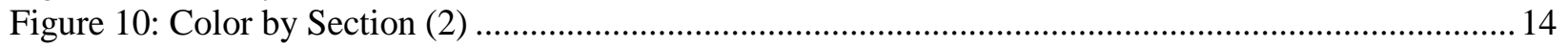

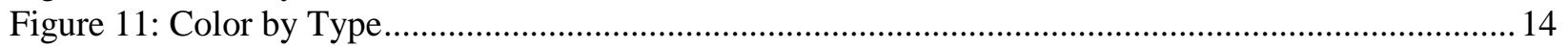

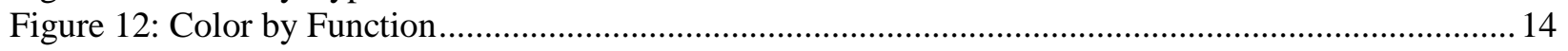

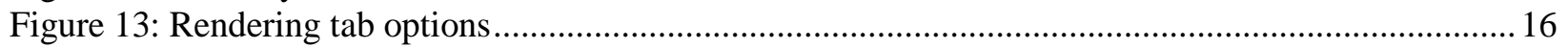

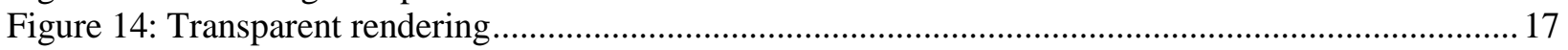

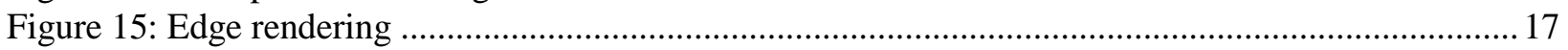

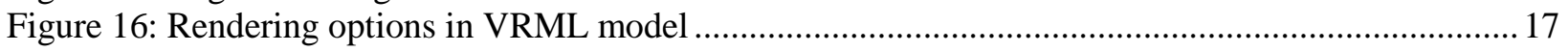

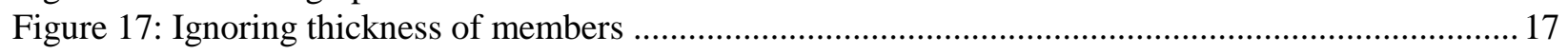

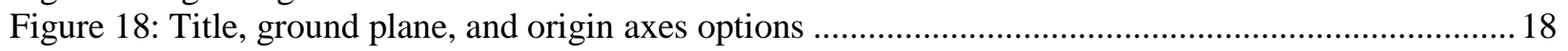

Figure 19: VRML model with title, ground plane, and origin axes ..................................................... 18

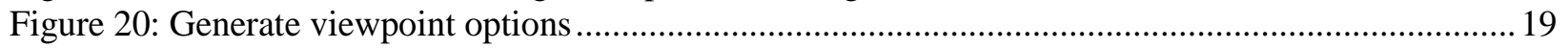

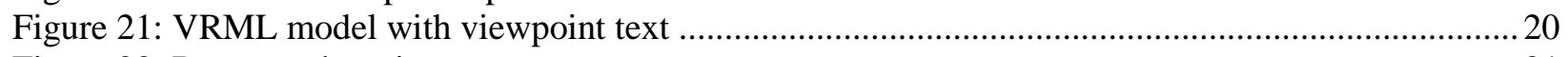

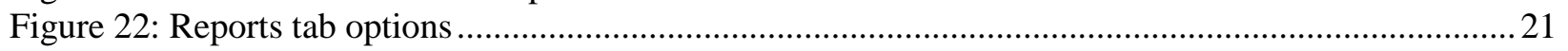

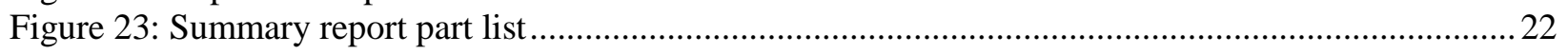

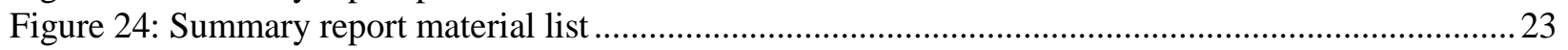

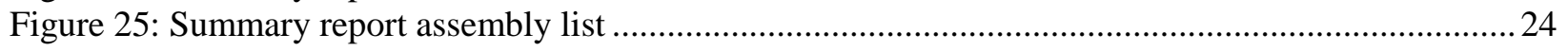

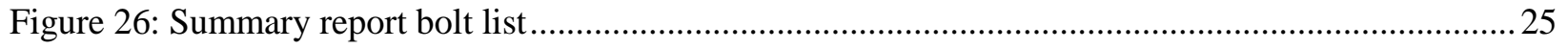

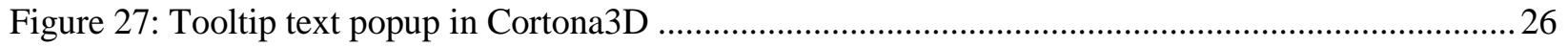

Figure 28: Tooltip text popup in Cosmo Player..................................................................................26

Figure 29: Tooltip text popup with assembly information in a separate web browser window .................22

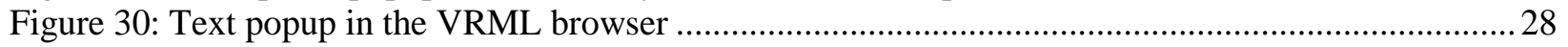

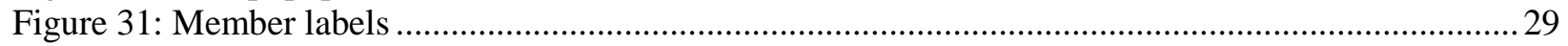

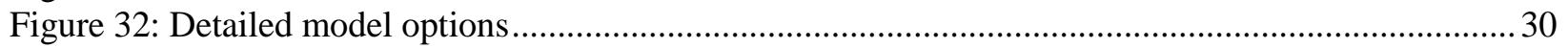

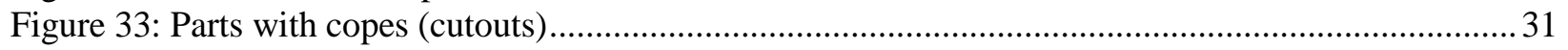

Figure 34: Parts with bolts, nuts, washers, holes, welds, shear stud .................................................. 32

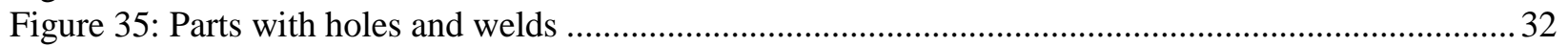

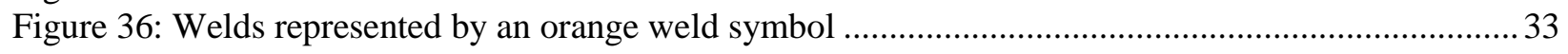

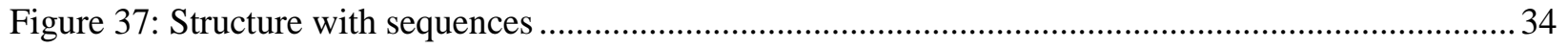

Figure 38: Structure with non-main parts and plates excluded ................................................................ 35

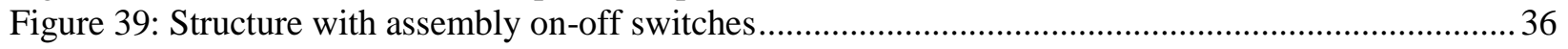

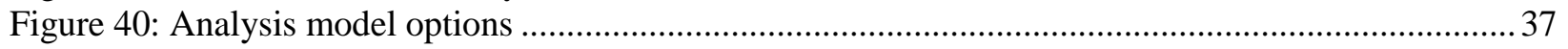

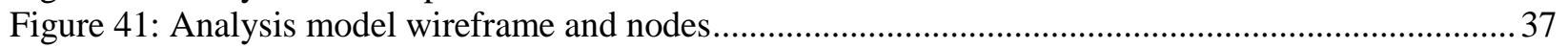

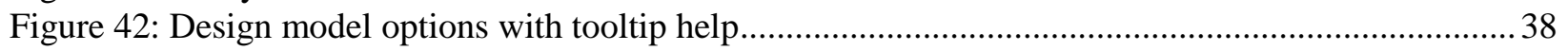

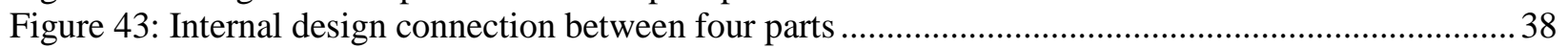

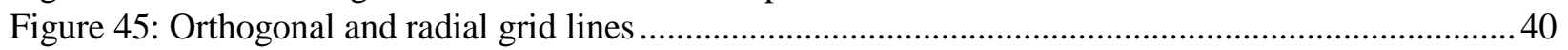

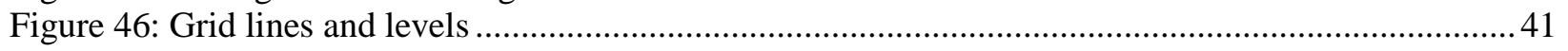

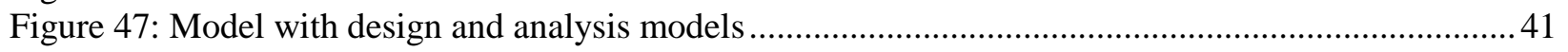

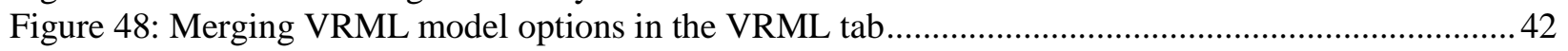

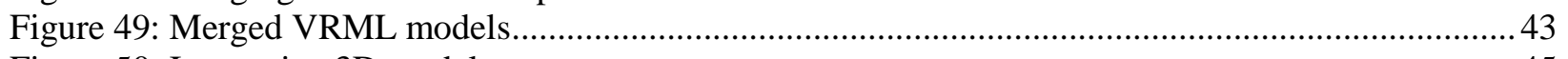

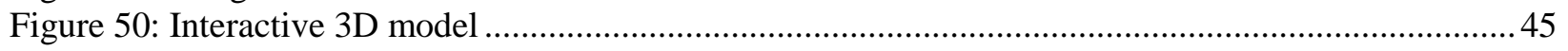




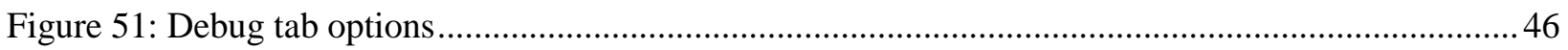

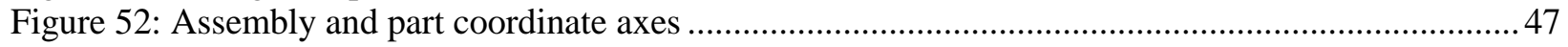

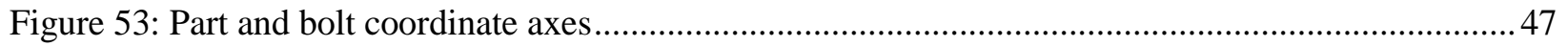

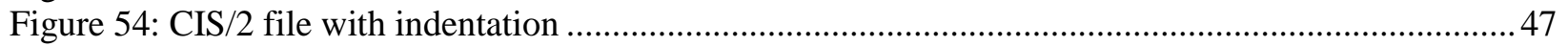

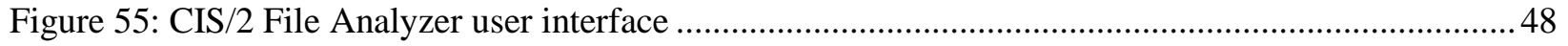

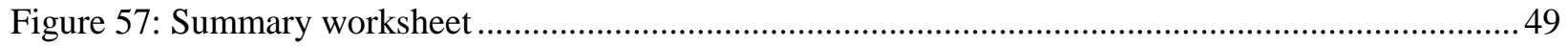

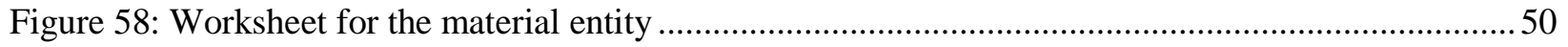

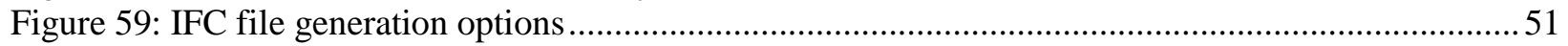

Figure 60: Structure colored by IFC type (element representation) ...................................................5

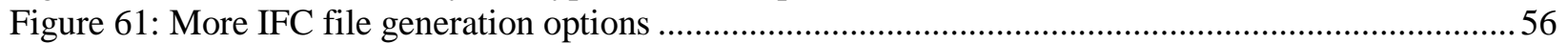

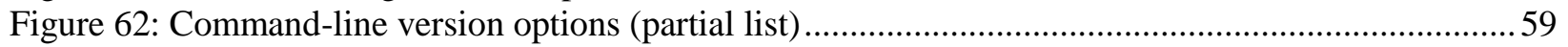

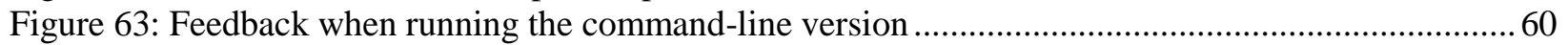

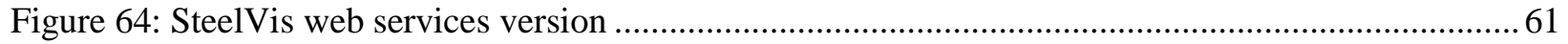





\section{Introduction}

The CIMsteel Integration Standards (CIS/2) [1,2] are the product model and electronic data exchange format for structural steel project information. CIS/2 is intended to enable a seamless and integrated flow of information among all parties of the steel supply chain involved in the design, analysis, engineering, fabrication, and construction of steel framed structures. The CIS/2 standard covers everything from nuts and bolts to materials and loads to frames and assemblies. Structures can be represented as design, analysis, or detailed models. Detailed models are also known as manufacturing, physical, or fabrication models. CIS/2 has been implemented as a file import and/or export capability in many steel design, analysis, engineering, fabrication, and construction software packages.

Using SteelVis as a CIS/2 file viewer provides a way to visually verify the information in a CIS/2 file and to provide feedback about many of the attributes of the structural steel model. To visualize a CIS/2 file, SteelVis generates a VRML (Virtual Reality Modeling Language) file [3, 4] which can be viewed in a web browser VRML plugin, VRML viewers, or other software that can display VRML files.

Using SteelVis to translate a CIS/2 file into an Industry Foundation Classes (IFC) file [5] allows information about a steel structure to be imported into Building Information Modeling (BIM) software that can only import IFC files and not CIS/2 files. The capability to import the CIS/2 model into BIM software allows for coordination between the structural steel model and other parts of the structure such as concrete and mechanical and electrical systems. IFC is the product model and electronic data exchange format to facilitate interoperability between software applications in the building industry. It is developed by buildingSMART [6], formerly known as the International Alliance for Interoperability.

The CIS/2 to VRML Translator was first developed in 2000 as a command-line program that ran on a Silicon Graphics workstation. In 2001, the web services version of the translator was made available online. In 2003, the Windows version with a graphical user interface was developed. In 2005, the capability to translate from a CIS/2 file to an IFC file was added to SteelVis. This User's Guide for SteelVis was written in 2011.

\section{Getting Started}

\subsection{Installing SteelVis}

The download request form for SteelVis can be found at http://ciks.cbt.nist.gov/cgi-bin/ctv/ctv_request.cgi. After submitting the download request, instructions about where to download SteelVis will be provided. The information is also emailed to the requestor. SteelVis is downloaded as a zip file named CTV. zip. The file is named CTV for $\underline{\text { CIS/2 }} \underline{\mathbf{T}} \mathbf{\text { VRML. }}$

The installation process does not require anything more than unzipping the file CTV.zip. This will create a directory named CTV containing six files:

1. CIS2_VRML.exe - SteelVis graphical user interface (GUI) version

2. ctv . exe - SteelVis command-line version, no GUI, (section 10 below)

3. ctv-64. exe - Version of ctv. exe that runs only on 64-bit computers

4. CIS2_Excel.exe-CIS/2 File Analyzer generates an Excel spreadsheet from a CIS/2 file, (section 8.3 below)

5. SteelVis-README-FIRST.pdf - A brief introduction to SteelVis

6. SteelVis-Users-Guide.pdf - This user's guide 
There are no restrictions as to where the CTV directory is located in the computer's file system, however, the four executable files and the User's Guide in the CTV directory should not be moved from the directory.

\subsection{Running SteelVis}

SteelVis can run on any Windows 7, XP, or Vista computer. There are no minimum system requirements for SteelVis although as with any computer program, more memory, a fast computer processor, and more graphics memory is better. SteelVis (CIS2_VRML . exe) is a 32-bit application. The size of the CIS/2 file that can be translated by SteelVis depends on the amount of computer memory and SteelVis options selected. CIS/2 files greater than approximately $200 \mathrm{MB}$ might require using the 64-bit command-line version of SteelVis (ctv-64 . exe) and a computer with more than 2 GB of memory.

To run SteelVis, simply double click on the icon for CIS2_VRML. exe. The first time SteelVis is run several setup functions are performed:

1. The user is asked if a shortcut to SteelVis can be created in the Start Menu and if an icon for SteelVis can be placed on the Desktop. The SteelVis icon is a cartoon representation of an Ibeam. Creating the shortcut and icon facilitates running SteelVis without having to remember where it is installed. A shortcut to SteelVis is also created in the CTV directory.

2. If more than one web browser is installed on the computer, the user is asked to select the default web browser to use with SteelVis. The selected web browser should be the web browser where a VRML plugin is already or will be installed. This selection can be changed later in the Browsers menu of SteelVis (section 3.1.2 below). SteelVis detects if the following web browsers are installed on the computer: Internet Explorer, Firefox, Google Chrome, Opera, and Safari.

3. A welcome dialog is displayed that indicates that the VRML Plugin Detector will run after clicking OK. The VRML Plugin Detector is described in section 2.3 below.

4. A file SteelVis_options.dat is created in the user's home directory that stores the current state of the SteelVis options. Do not edit this file.

Also, the first time SteelVis is run, two directories html and sample are created in the CTV directory and several utility and sample CIS/2 files are written to those directories. The second time SteelVis is run, the Disclaimers dialog is displayed.

The sample directory contains seven CIS/2 files:

- cis2 analysis.stp - analysis model, display options are described in section 6.2 below

- cis2_design.stp - design model, display option are described in section 6.3 below

- cis2_detailed.stp - detailed model, shown in Figure 37 below, display options are described in section 6.1 below

- cis2_curved.stp - detailed model of curved beams, see section 7.4 below

- cis2ifc test1.stp, cis2ifc test2.stp-use to test IFC Shape Representation and Cardinal Point, (sections 9.1 and 9.6.5 below)

- cis2ifc_test3.stp - all section types, see Figure 7 below

The sample CIS/2 files can be used to understand and test: (1) various features of SteelVis, (2) how a VRML plugin or viewer displays a VRML model, (3) how a CIS/2 file is translated to an IFC file, and (4) how an IFC application imports and displays the IFC file. 


\subsection{Installing a VRML Plugin}

To visualize a CIS/2 file, SteelVis generates a VRML (Virtual Reality Modeling Language) file. VRML is a standard file format that is used to display 3D models in a web browser VRML plugin or other VRML viewer. Without a VRML plugin or viewer, the 3D interactive model of the CIS/2 file cannot be displayed.

The VRML Plugin Detector runs in the web browser that was selected in step 2 above. It determines which, if any, VRML plugin is installed in the web browser. It does not detect other VRML viewers. A security warning might be displayed when running the VRML Plugin Detector in Internet Explorer. In this case click on the warning and select "Allow Blocked Content...".

If a VRML plugin is installed, then a sample VRML model, which was generated from a CIS/2 file, is also displayed. If a VRML plugin is not detected, then a list of VRML plugins that work with SteelVis is provided. Currently, those VRML plugins are:

- BS Contact - http://www.bitmanagement.com/en/products/interactive-3d-clients/bs-contact

- Cortona3D Viewer - http://www.cortona3d.com/Products/Cortona-3D-Viewer.aspx

- Cosmo Player - http://cic.nist.gov/vrml/cosmoplayer.html

- Flux Player - http://mediamachines.wordpress.com/flux-player-and-flux-studio/

- Octaga Player - http://octagavs.com/softwarem/octaga-player

Select one of the VRML plugins and follow the instructions that come with the VRML plugin to install it in the web browser. All of the VRML plugins can be installed in Internet Explorer and Firefox and some can also be installed in Google Chrome, Opera, and Safari. Some of the VRML plugins will install in the 64-bit version of Internet Explorer. Although Cosmo Player is available from the NIST web site, it was not developed at NIST.

A license is available for purchase with BS Contact, Cortona3D Viewer, and Octaga Player. The license removes the product logo from the VRML browser window. BS Contact, Flux Player, and Octaga Player also come with standalone VRML viewers that do not require a web browser to run in. They can also be used by SteelVis to display VRML models (section 3.1.2 below).

All of the VRML plugins have been tested to ensure that they work correctly with SteelVis. The main difference between the VRML plugins is how the user interacts with the VRML model that is displayed. All of the VRML plugins allow the user to rotate, pan, and zoom the VRML model; however, each uses different functionality for the mouse buttons and menu items.

\subsection{Translating a CIS/2 File}

After a VRML plugin or viewer is installed, a CIS/2 file can be translated into a VRML model by opening up a CIS/2 file from the File menu (section 3.1.1 below) and clicking on the Translate and Display button (section 3.4 below).

The display options in the user interface that affect the appearance of the VRML model are described in the sections 3 through 8 . Every time the display options are changed, the CIS/ 2 file must be translated and displayed again. The VRML model cannot be updated without translating the CIS/2 file. Selecting some display options might enable or disable other display options.

If the CIS/2 file is named mycis2file.stp, then the resulting VRML file will be named mycis2file_ctv.wrl. The file extension for VRML files is "wrl". 
SteelVis cannot translate an IFC file into a CIS/2 file, although some research related to this topic was done at Georgia Tech [7]. It also cannot translate a VRML file into a CIS/2 file or IFC file. However, there is limited support for translating some types of CIS/1 and DSTV files to VRML files.

\subsection{Viewing the VRML Model}

Once the VRML model is displayed in the VRML plugin or viewer, the user can interactively navigate around the 3D model. How the user interacts with the model depends on the VRML plugin. For some of the display options, small round buttons will appear in the VRML browser that the user can click on to toggle on and off parts of the model. The functionality of the buttons depends on the VRML plugin.

A security warning might be displayed when trying to view a VRML model with a VRML plugin in Internet Explorer. In this case click on the warning and select "Allow Blocked Content...".

\subsection{Uninstalling SteelVis}

SteelVis can be uninstalled by manually deleting several files and directories. Deleting the CTV directory will remove the SteelVis executable and associated files. In a user's home directory, the file SteelVis_options.dat can be deleted. Finally, the desktop icon for SteelVis can be deleted. 


\section{User Interface}

Figure 1 shows the SteelVis user interface running on a Windows XP computer. The appearance and functionality of the interface on a Windows 7 or Vista computer is similar.

At the top of the user interface is the Menu Bar with the File, Browsers, Websites, and Help menus.

Below that is the Tabs Bar with one tab for the Status window and the other tabs that provide access to all of the display options. Below that is the Status window that shows the text feedback when SteelVis is running. Clicking on one of the other tabs will switch to the user interface for that tab. At the bottom is a row of four buttons and four images.

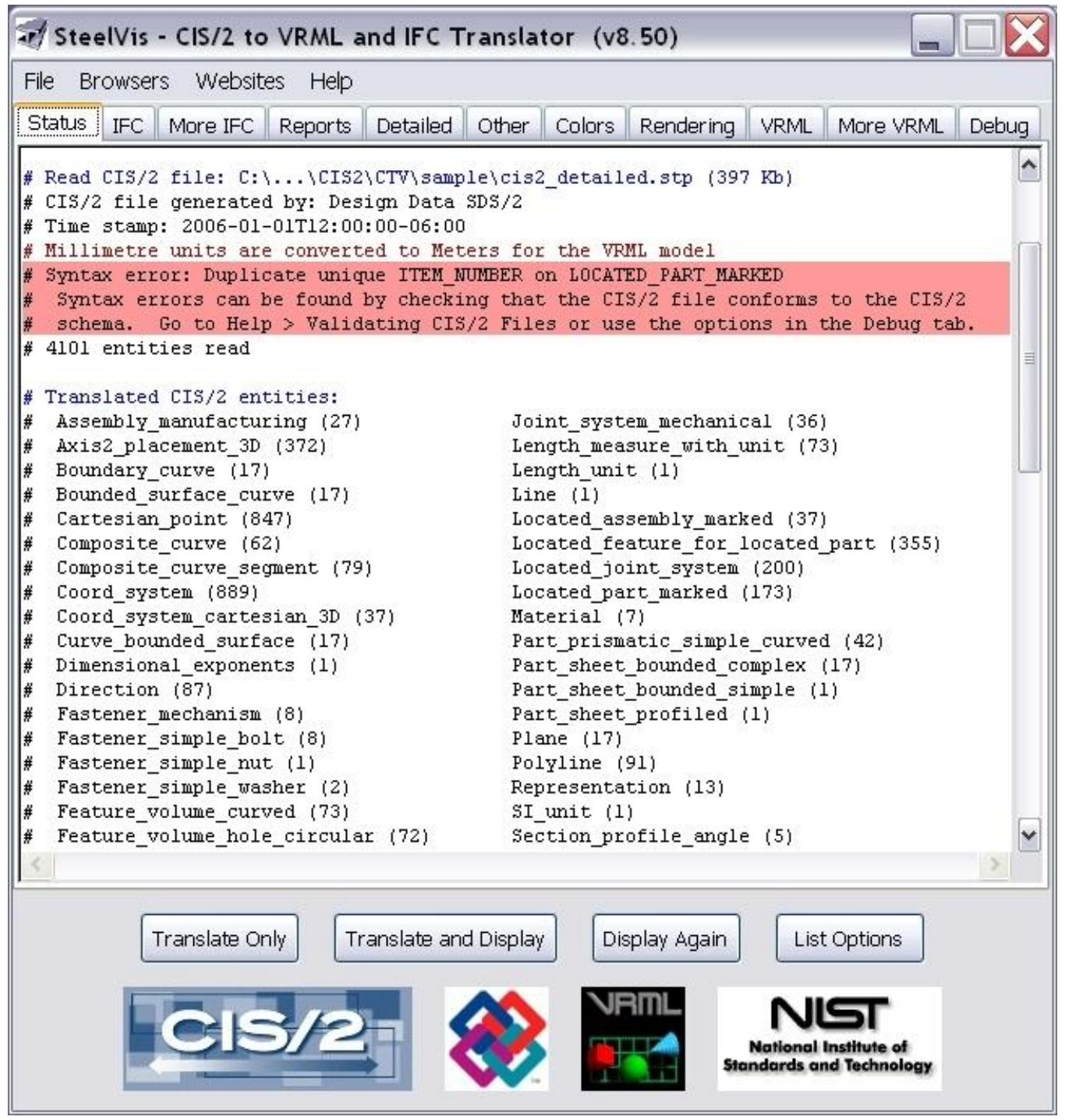

\section{$<$ Menu Bar $<$ Tabs Bar}

$<$ Status Tab and display options

Figure 1: SteelVis user interface

\section{$<$ Translate Buttons}




\subsection{Menu Bar}

\subsubsection{File Menu}

From the File menu, shown in Figure 2, the user can select which CIS/2 file to open for translation. Multiple CIS/2 files can also be processed at one time. The user will be asked to select a directory to search for CIS/2 files. The search for multiple files can be restricted to only the selected directory or to include all subdirectories.

Below the first solid line in the File menu is a list of up to 20 of the most recently translated CIS/2 files which can be opened directly. The function keys F1 and F2 can be used to Translate and Display or Only Translate, respectively, the CIS/2 file that was most recently translated. In addition to using the functions keys F1 and F2, the Translate and Display and Translate Only options can be selected from the bottom of the File menu.

Below the list of CIS/2 files is a link to download a zip file of 25 sample CIS/2 files. The zip file also contains X3DOM files that can be displayed without a VRML plugin or viewer (section 7.7 below) and Excel spreadsheets (section 8.3 below) generated from the sample CIS/2 files. Other sample CIS/2 files are located in the sample subdirectory of the CTV directory where SteelVis is installed. An explanation of those files is found in section 2.2 above.

The Export Preferences option allows the user to export the current state of the SteelVis display options to a user-defined file. That file can be imported to SteelVis with the Import Preferences option. Preferences are a useful way to save and restore commonly used settings of SteelVis display options.

Any VRML or IFC file can be displayed again with the Redisplay Any File option which can also be accessed with the F3 function key.

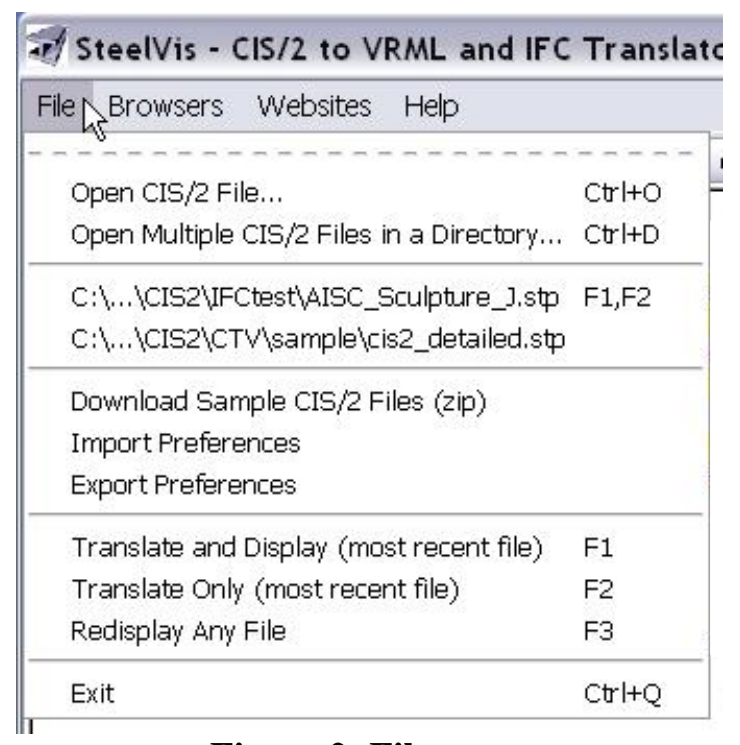

Figure 2: File menu

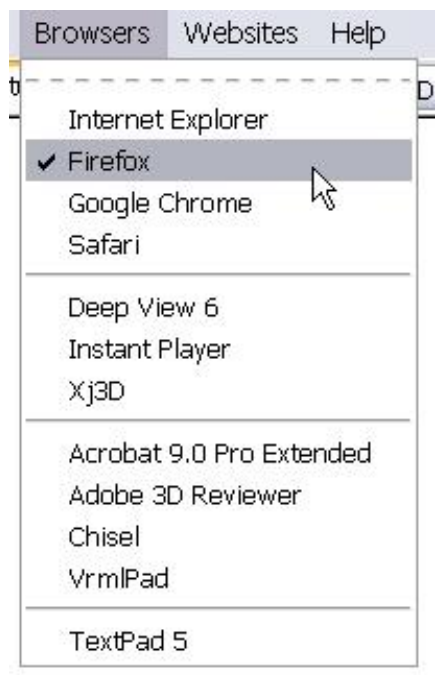

Figure 3: Browsers menu 


\subsubsection{Browsers Menu}

The Browsers menu, shown in Figure 3, is used to select which web browser, viewer, or program will display the VRML file when the Translate and Display or Display Again buttons (section 3.4 below) are clicked. It shows a list of programs installed on the user's computer where a VRML file might be displayed. Programs are listed in the Browsers menu if they are installed in their default locations such as $\mathrm{C}$ : Program Files or if there is a shortcut to them on the desktop. The list of web browsers will always contain all web browsers found on the computer regardless if they have a VRML plugin installed in them. At a minimum, the Browsers menu will always contain Internet Explorer and the WordPad text editor.

If a VRML file has already been generated, the user can select a different program in the Browsers menu and then click the Display Again button (section 3.4 below) to display the VRML file in the selected program.

In the example list of browsers, Deep View 6, Instant Player, Xj3D, and Adobe 3D Reviewer are viewers that display VRML files. Acrobat 9.0 Pro Extended is software used to embed a VRML file in a PDF document. Chisel is a VRML file optimizer, VrmlPad is a VRML file editor, and TextPad is a text editor similar to WordPad. VRML viewers, such as BS Contact and Flux Player that come with their associated VRML plugins, can also appear in the Browsers menu.

\subsubsection{Websites Menu}

The Websites menu, shown in Figure 4, provides links to useful resources related to SteelVis, VRML, CIS/2, and IFC.

\subsubsection{Help Menu}

The Help Menu, shown in Figure 5, has four sections. In the first section, "What's New" displays information in the Status tab about new features in SteelVis. The "What's New" information is automatically displayed every time a new version of SteelVis is run.

"Check for Update" opens up a web page that checks for the latest version of SteelVis. Follow the instructions on that web page to download a new version of SteelVis if one is available. The "Check for Update" feature runs automatically if an update hasn't been checked for in the last 30 days.

"VRML Plugin Detector" runs the same check for a VRML plugin described in section 2.3 above.

Selecting the other topics in the Help menu displays information related to them in the Status tab.

Help is also available in the form of tooltips related to the display options in the tabs. Holding the mouse over any text in a tab for a second or two will display a tooltip. An example of tooltip help in the Detailed tab is shown in Figure 6.

Previous to this user's guide, the Help menu and tooltips were the only documentation available for SteelVis. 


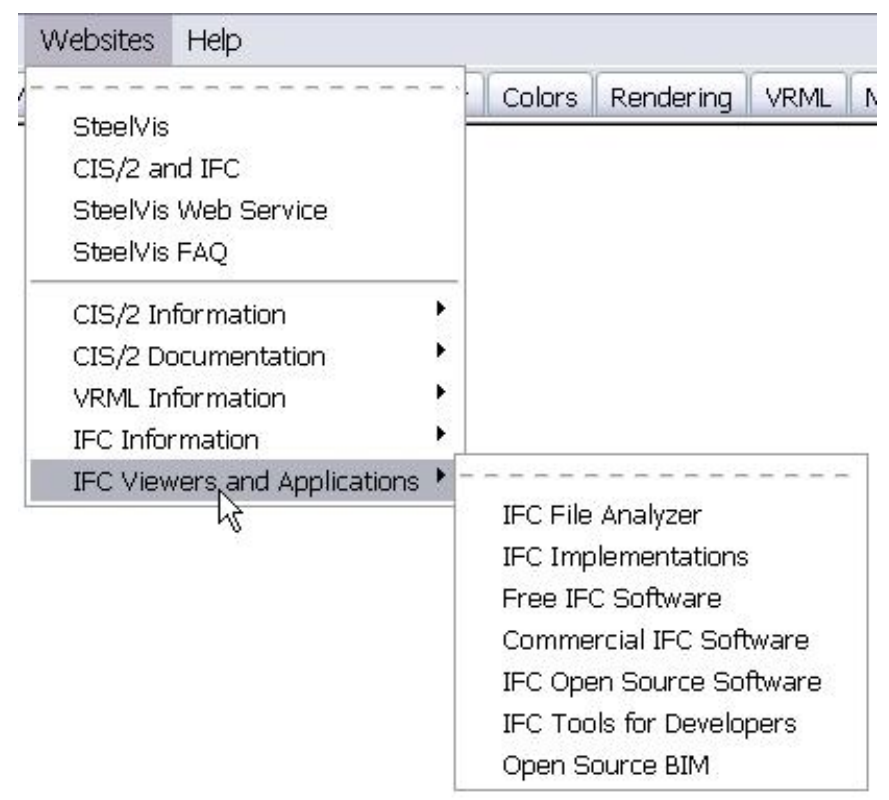

Figure 4: Websites menu

\begin{tabular}{l} 
Help \\
What's New \\
Check for Update \\
VRML Plugin Detector \\
\hline Overview \\
Short User's Guide \\
IFC Overview \\
Report Generation \\
User-Defined Viewpoints \\
CIS/2 Test Files \\
Validating CIS/2 Files \\
Generate Spreadsheet \\
Known Problems \\
\hline Generate X3DOM or X3D Files \\
Convert to Google SketchUp \\
Embed VRML in PDF \\
\hline Reporting Errors \\
Disclaimers \\
About \\
\hline
\end{tabular}

Figure 5: Help menu

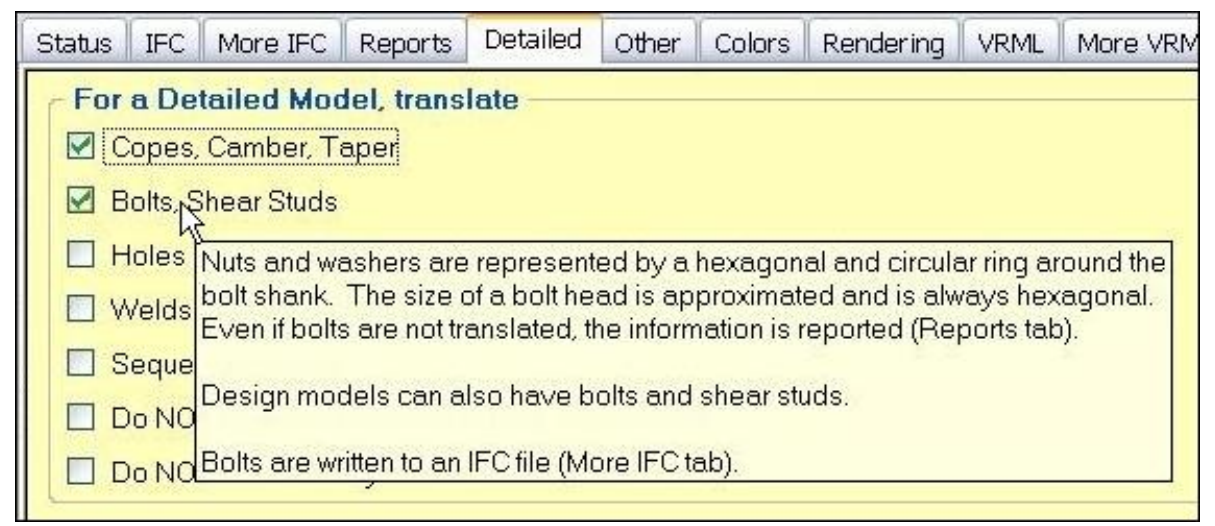

Figure 6: Example of tooltip help

\subsection{Tabs Bar}

The tabs bar is located directly below the menu bar in Figure 1. Clicking on a tab will switch from the current tab to the selected tab. Except for the Status tab, the other tabs contain all of the SteelVis display options similar to Figure 6 above. The display options in the tabs will be described in the sections below, in the context of their function rather than in the order of the tabs.

\subsection{Status Tab (Feedback)}

The Status tab displays important feedback during the translation of a CIS/2 file. The information should not be ignored as it provides useful insight as to why the resulting VRML model or IFC file may not 
appear as expected. A brief example of the information in the Status tab is shown in Figure 1. The types of messages that appear in the Status tab will depend on the CIS/2 file that is translated.

The following is the general sequence of most of the information that appears in the Status tab when a CIS/2 file is translated:

- Name of the program that generated the CIS/2 file and the time stamp in the CIS/2 file

- Progress is indicated by a counter that increments by 5000 while the lines of the CIS/2 file are read

- Total CIS/2 entities read

- A list of all translated CIS/2 entities including the number of each entity

- A list of all ignored CIS/2 entities that includes all entities not recognized by SteelVis or those that do not have to be processed depending on the selected display options

- CIS/2 model type: Design, Analysis, or Detailed or any combination of the three

- Messages about processing of units, lengths, coordinates, sections, copes, bolts, holes, openings, and assemblies depending on the CIS/2 model type. Particular attention should be paid to the messages about "Processing: sections" (section 3.3.2 below).

- Messages about generating the VRML model

- Messages about generating the Summary Report if the option is selected, (section 5.1 below)

- Messages about generating an IFC file if the option is selected, (section 9 below)

- Names of the files that were generated and their size

- Current configuration of the display options

\subsubsection{Error and Warning Messages}

The text or background color of the messages in the Status tab has significance:

- Messages with a yellow background or red text are errors, warnings, or informational

- Messages with a light blue background are related to generating an IFC file, (section 9 below)

- Messages with a red background are syntax errors in the CIS/2 file and indicate a nonconformance to the CIS/2 schema, however, SteelVis does not check for all syntax errors. Conformance to the schema and complete syntax checking can be performed with other tools, (section 8.4 below). Use the option for 'More warning and error messages' in the Debug tab to report specific CIS/ 2 entities that are the cause of some syntax errors.

Any of the error or warning messages might affect how the CIS/2 file is translated into a VRML model or IFC file. However, any of the error or warning messages, including syntax errors, does not necessarily imply that the CIS/2 file cannot be used successfully to exchange information with other software applications.

\subsubsection{Processing of Sections}

The information in the Status tab regarding processing of sections can contain information about a common source of problems regarding the visual appearance the resulting VRML model. A CIS/2 file can specify the explicit dimensions of sections such as the depth, width, web thickness, and flange thickness for an I-beam and other section types. In this case, there is no ambiguity as to what the section dimensions are and how parts with those sections will appear in the VRML model. If there are entities such as Section_profile_i_type, Section_profile_angle and other similarly named entities in the Status tab feedback related to the "Translated CIS $/ \overline{2}$ entities", then these are CIS/2 entities that explicitly specify the dimensions of those sections. 
However, it is also valid for a CIS/2 file to only specify the section designator such as W14X120 without any section dimensions. If the entity Section_profile is present, then only the section designator is used to specify the section. In this case, an internal lookup table is used to find the dimensions for the section. The lookup table contains the section dimensions for over 4400 of the most commonly used sections from around the world. Also included in the lookup table are equivalent names for the same sections. For example, a 75PFC and 75X40X6PFC refer to the same section and have the same dimensions. Similarly, an HEA100 and HE100A refer to the same section and a W14X61 and W14*61 also refer to the same section. The section designator that is used in the CIS/2 file is completely dependent on the software that generated the CIS/2 file.

Some section types, such as angles and rectangular sections, are not included in the lookup table and the dimensions can usually be parsed directly from the section designator. For example, an L250X125X13 is interpreted as an angle that is $250 \mathrm{~mm}$ deep, $125 \mathrm{~mm}$ wide, and has a thickness of $13 \mathrm{~mm}$.

In the VRML model, for all sections, webs and flanges always have constant thickness, and the fillets between webs and flanges are ignored. Leg slope for angles is also ignored.

If the dimensions for a section cannot be found in the lookup table then the section dimensions and section type (I-beam, angle, channel, etc.) will be parsed or approximated from the section designator. However, sometimes that approximation is wrong and the resulting VRML model will look incorrect for those sections that are approximated. For example, if the section designator is only given as "SSMM-12", there is no way to know what the section dimensions or type is. In this case a rectangular section is used. There can also be ambiguities when interpreting the units of the numbers in the section designator. If a number in a section designator (for example the "12" in "SSMM-12") is interpreted to be in inches when it is actually millimeters, then the section will appear much too large.

In the Status tab, if the CIS/2 file contains only explicit section dimensions, then there is no feedback for the "processing of sections". However, if the CIS/2 file contains any sections that are specified only by the section designator, then the feedback in the Status tab can contain any or all of the following information:

- Number and name of sections found in the lookup table

- Number and name of sections that were parsed from the section designator

- Number and name of sections that were approximated from the section designator and their approximated dimensions and type

- Number and name of sections that were not recognized

Sections that are parsed, approximated, or not recognized might look wrong in the VRML model.

The location of the longitudinal axis of a part relative to its cross section is known as the cardinal point in CIS/2. The most common cardinal points such as top-of-steel or center of the section are processed, however, cardinal points for double sections, joists, and edge-defined sections are ignored. If the cardinal point is ignored or approximated, then the part will appear slightly offset from its actual location. 


\subsubsection{Section Types}

Figure 7 shows an example of most of the different types of sections that SteelVis can write to a VRML file. They are specified with explicit section dimensions or from dimensions found in the lookup table. The CIS/2 file for this example is cis2ifc_test3.stp in the sample directory (section 2.2 above).

Row-by-row starting from the bottom left, the section types are:

- I-beam, T-beam, channel, angle, cold-formed channel

- Solid circular, hollow circular, solid rectangular, hollow rectangular, cold-formed zee

- Tapered beam, plate, double channel, double angle, joist

- Bent plate, corrugated decking, curved beam

The representation of joists is only an approximation because the only information in a CIS/2 file about a joist section is its depth. All joists are represented by double angles for the top and bottom chords and rectangular bars for the web.

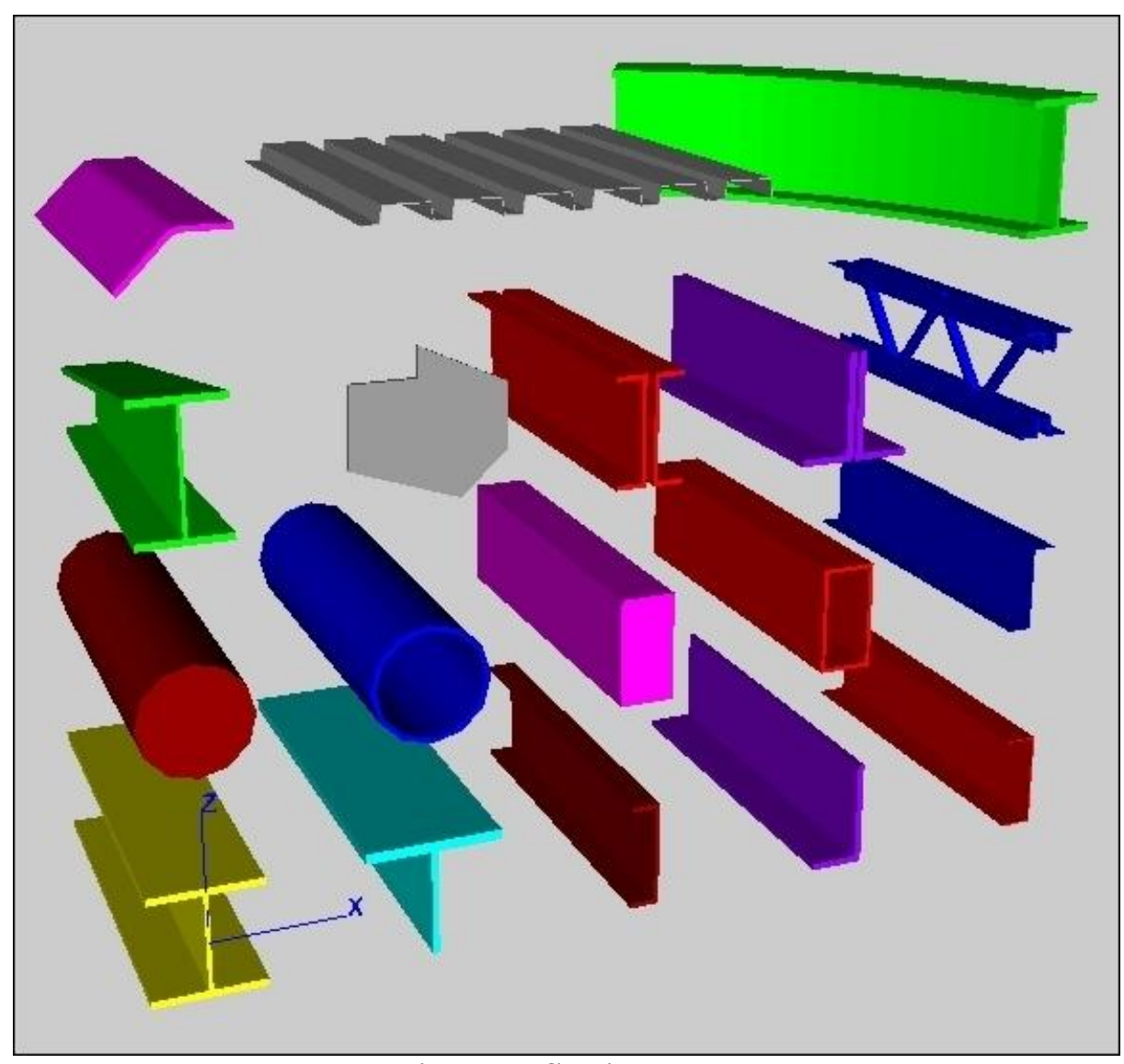

Figure 7: Section types 


\subsection{Translate Buttons}

The translate buttons are located at the bottom of the user interface, as shown in Figure 1, and have the following functions:

- Translate Only - Translate the CIS/2 file and generate a VRML model, however, it is not displayed. Optionally, an IFC file can also be generated, (section 9 below).

- Translate and Display - Same as Translate Only but the VRML model is displayed in whatever web browser or viewer is selected in the Browsers menu (section 3.1.2 above). Optionally, an IFC file can also be generated (section 9 below).

- Display Again - Redisplay the VRML model in whatever web browser or viewer is selected in the Browsers menu (section 3.1.2 above). This is useful to display the same VRML model in different VRML plugins, viewers, or editors without have to retranslate the CIS/2 file.

- List Options - The current display options are listed in the Status tab.

Below the Translate buttons are four images related to CIS/2, VRML, IFC, and NIST. The images are links to web sites associated with the image. 


\section{Visual Appearance}

Many options are available to change the visual appearance of the VRML model. Most of the settings for those options are found in the Colors, Rendering, and Other tabs. Some of the options are applicable only to certain types of CIS/2 models or features in a CIS/2 file. Some options are disabled or enabled depending on the selection of other options. Every time the display options are changed, the CIS/2 file must be translated and displayed again. The VRML model cannot be updated without translating the CIS/2 file.

\subsection{Color Options}

The options to change the colors assigned to members in the VRML model are found in the Colors tab as shown in Figure 8.

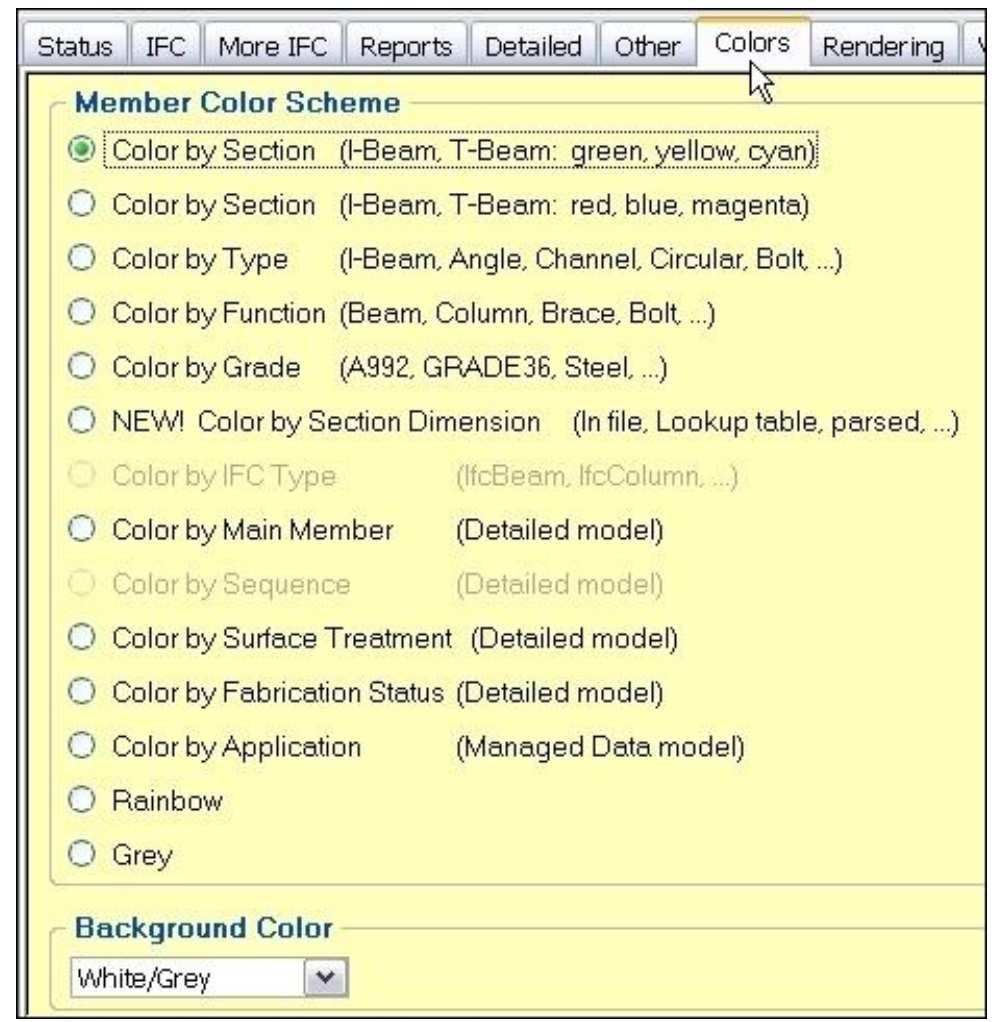

Figure 8: Colors tab options

Figures 9 through 12 are examples of the same VRML model colored using the first four color options. Figure 9 shows the model using the first Color by Section option. For this option all I-beams and Tbeams are green, dark green, yellow, dark yellow, or cyan (light blue). All other sections except plates are red, blue, magenta, and purple. Bolts and welds are always orange. Anything considered to be a plate is always grey. Shear studs are always beige. Members with the same cross section always have the same color, however, members with the same color do not always have the same cross section. Figure 10 shows the model using the second Color by Section option. This is the same as the first option except that the color palette for the members is reversed.

Figure 11 shows the model using the Color by Type option which assigns colors to the members based on their cross section type, i.e. I-beam, angle, channel, etc. A legend shows the colors assigned to each type. Figure 12 shows the model using the Color by Function option which assigns colors to the members 
based on their function. How functions are specified for the members is completely dependent on the software that generated the CIS/2 file. If functions are assigned to members they are displayed in the Summary Report (section 5.1.1 below).

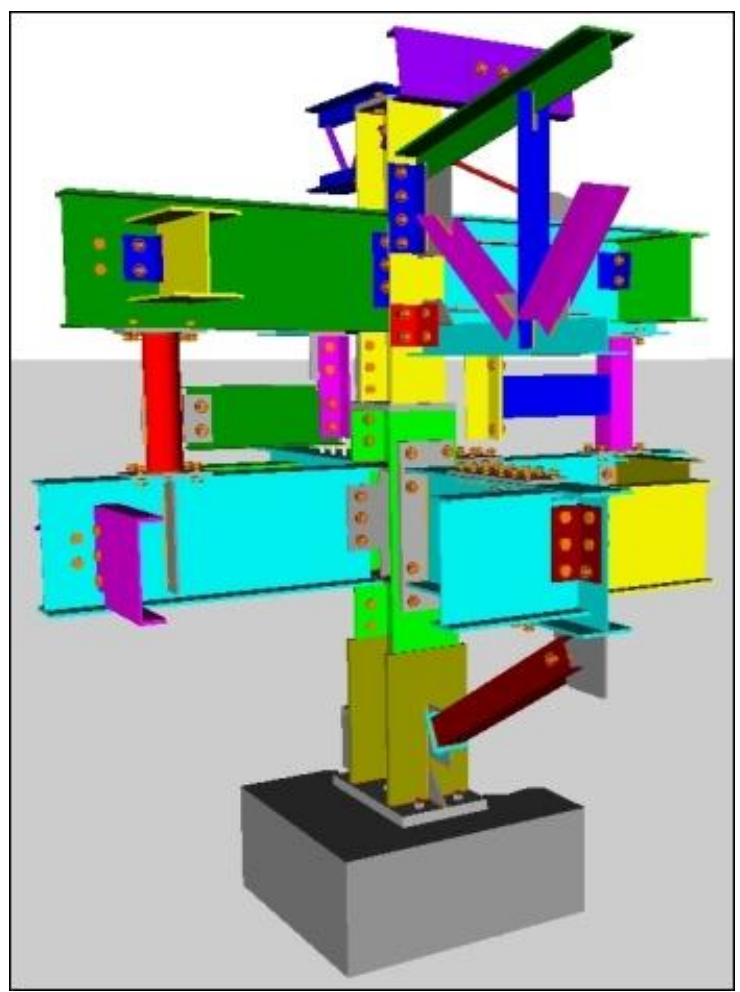

Figure 9: Color by Section (1)

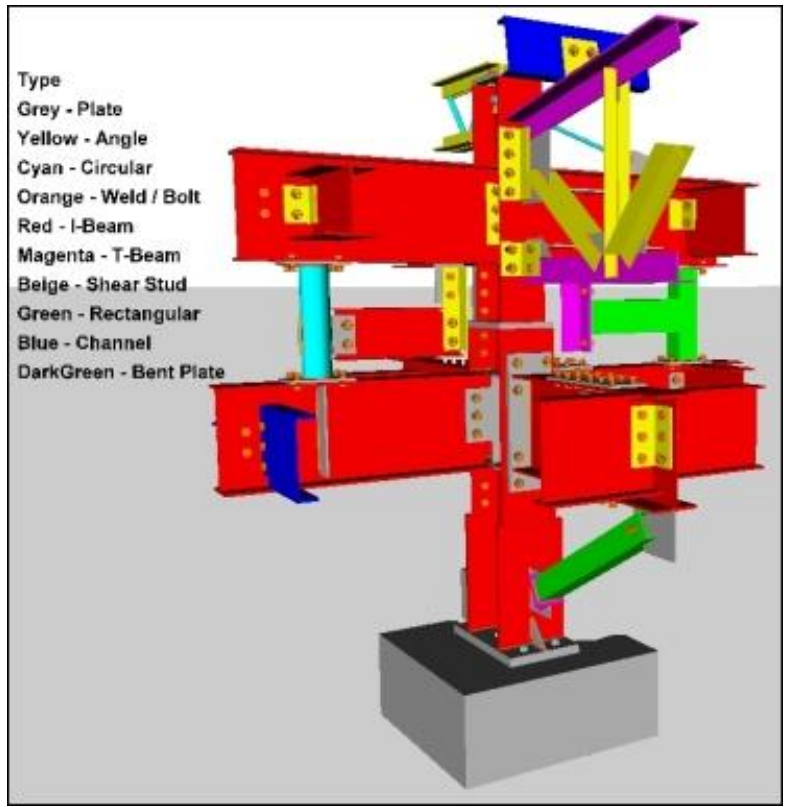

Figure 11: Color by Type

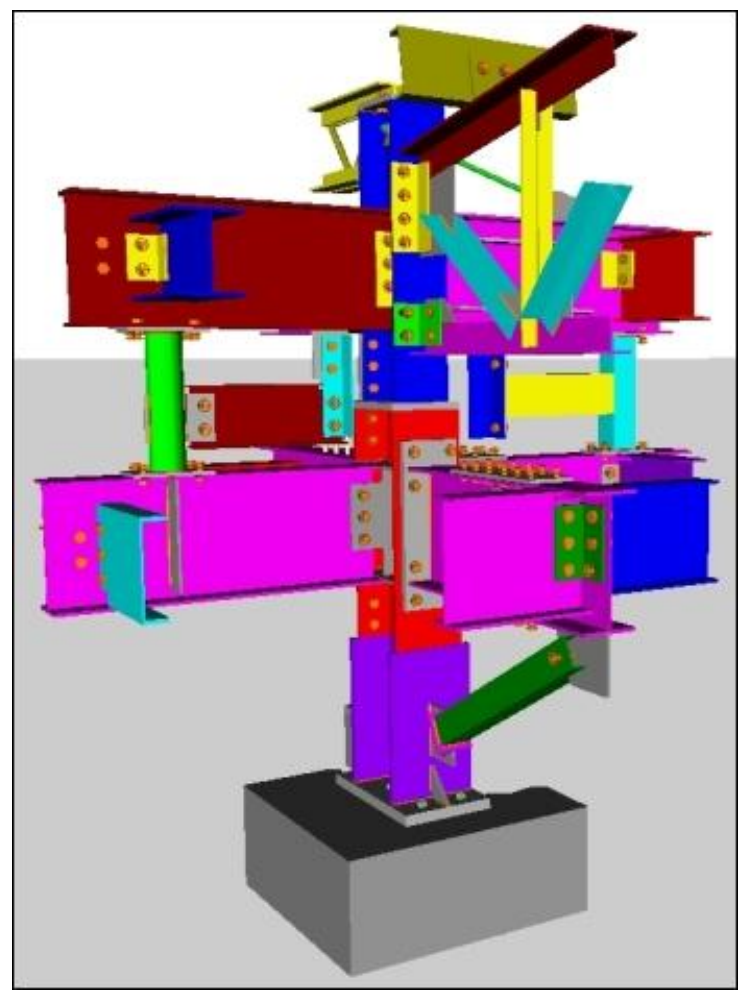

Figure 10: Color by Section (2)

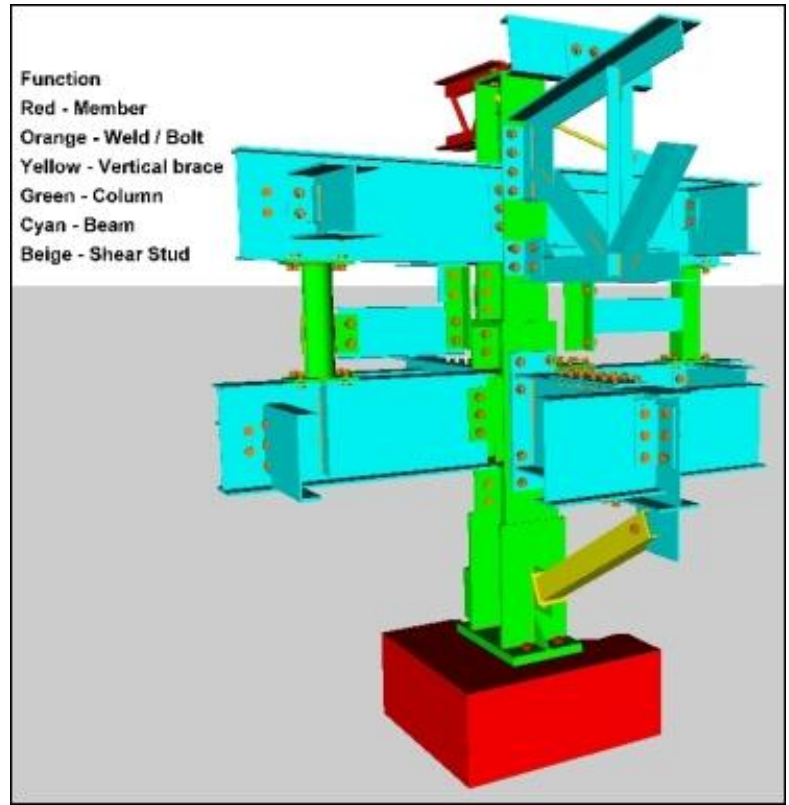

Figure 12: Color by Function 
The other options to color the VRML model are:

- Color by Grade - Similar to Color by Function except that the name of the material assigned to a member is used as the basis for the color.

- Color by Section Dimension - Color is assigned based on how the dimensions for a member's cross section are determined, (section 3.3.2 above).

- Color by IFC Type - Color is assigned based on the type of IFC entity used for a member, (section 9.2 below and Figure 60). This option is enabled when an IFC file is generated from the CIS/2 file.

- Color by Main Member - Some CIS/2 files differentiate between the main member of an assembly (e.g. a beam) and the other members in the assembly (e.g. angle brackets and gusset plates). All main members will be one color and the other members another color. CIS $/ 2$ files that contain main members use the entity Located_part_marked. These entities can be checked for in the Status tab in the "Translated CIS $/ 2$ entities" section.

- Color by Sequence - Color is assigned based on groupings of members by sequences or zones. All members in the same sequence or zone will have the same color. This option is enabled when sequences and zones are translated in a detailed model, (section 6.1.3 below and Figure 37).

- Color by Surface Treatment - Color is assigned to members based on their surface treatment. CIS/2 files with member surface treatment have the entities Coating and Surface_treatment.

- Color by Fabrication Status - Color is assigned to members based on their fabrication status. CIS/2 files with fabrication status information are not very common. If this option is selected and the CIS/2 file contains the necessary entities need to assign colors, then after processing of the CIS/2 file has started, a dialog will appear and the status parameter can be selected that will be used to color members.

- Color by Application - Color is assigned to members based on which software application created the member. CIS/2 files with application information are not very common.

- Rainbow - Color is assigned to members randomly.

- Grey - All members are colored grey.

Parts that cannot be assigned a color based on the selected color option will appear grey. If a color option is selected that does not apply to the type of CIS/2 model being translated, then the entire VRML model will appear grey and a message will appear in the Status tab.

\subsubsection{Background Color}

Four different background colors can be displayed in the VRML model: white/grey, white, black, and sky/ground. The white/grey background is used in all of the figures with VRML models. 


\subsection{Rendering Options}

The options to change how members are rendered in the VRML model are found in the Rendering tab as shown in Figure 13.

\begin{tabular}{|c|c|c|c|c|c|c|c|c|c|c|}
\hline Status & IFC & More IFC & Reports & Detailed & Other & Colors & Rendering & VRML & More VRML & Debug \\
\hline \multicolumn{4}{|c|}{ Rendering Options } & \multicolumn{7}{|c|}{ Cons } \\
\hline \multicolumn{11}{|c|}{ (9) Shaded only } \\
\hline \multicolumn{11}{|c|}{ O Transparent only } \\
\hline \multicolumn{11}{|c|}{ Edge only } \\
\hline \multicolumn{11}{|c|}{ Shaded, transparent edge, and wireframe (requires VRML with PROTOs, see More VRML tab) } \\
\hline \multicolumn{11}{|c|}{ Simplify VRML Model } \\
\hline \multicolumn{11}{|c|}{$\square$ By ignoring Thickness of: Webs, flanges, hollow sections } \\
\hline \multicolumn{11}{|c|}{$\square$ Byignoring Thickness of: Plates, bent plates, cold-formed parts, decking } \\
\hline \multicolumn{11}{|c|}{$\square$ With simple bolts, holes, and welds (lines instead of polygons) } \\
\hline
\end{tabular}

Figure 13: Rendering tab options

Figures 14 through 17 are examples of the same VRML model with displayed with different rendering options. In Figure 14, all of the members, bolts, holes, and welds are transparent. The anchor rods in the base of the structure are now visible. Figure 9 is the same model displayed with shaded (non-transparent) rendering.

In Figure 15, most of the members are displayed only by their edges. Bolts, holes, and welds are displayed only with lines. Bent plates, corrugated decking, and curved parts cannot be displayed by only their edges.

Figure 16 uses the fourth option in the Rendering tab for shaded, transparent, edge, and wireframe. This option requires the "VRML with PROTOs" option in the More VRML tab (section 7.9 below). The different rendering options can be selected directly in the VRML display. In this VRML model, there are also buttons to toggle on and off the bolts, holes, and welds. This rendering option is not recommended for very large CIS/2 models.

Figure 17 shows the VRML model with all three of the Simplify VRML Model options turned on. Ignoring the thickness of member greatly reduces the number of surfaces that have to be displayed in the VRML model. This is useful for very large CIS/2 models or when displaying the VRML model on slower computers. Bolts, holes, and welds are displayed only by lines. Since the base of the structure is modeled as a plate, it is displayed as a surface through the middle of the plate. 


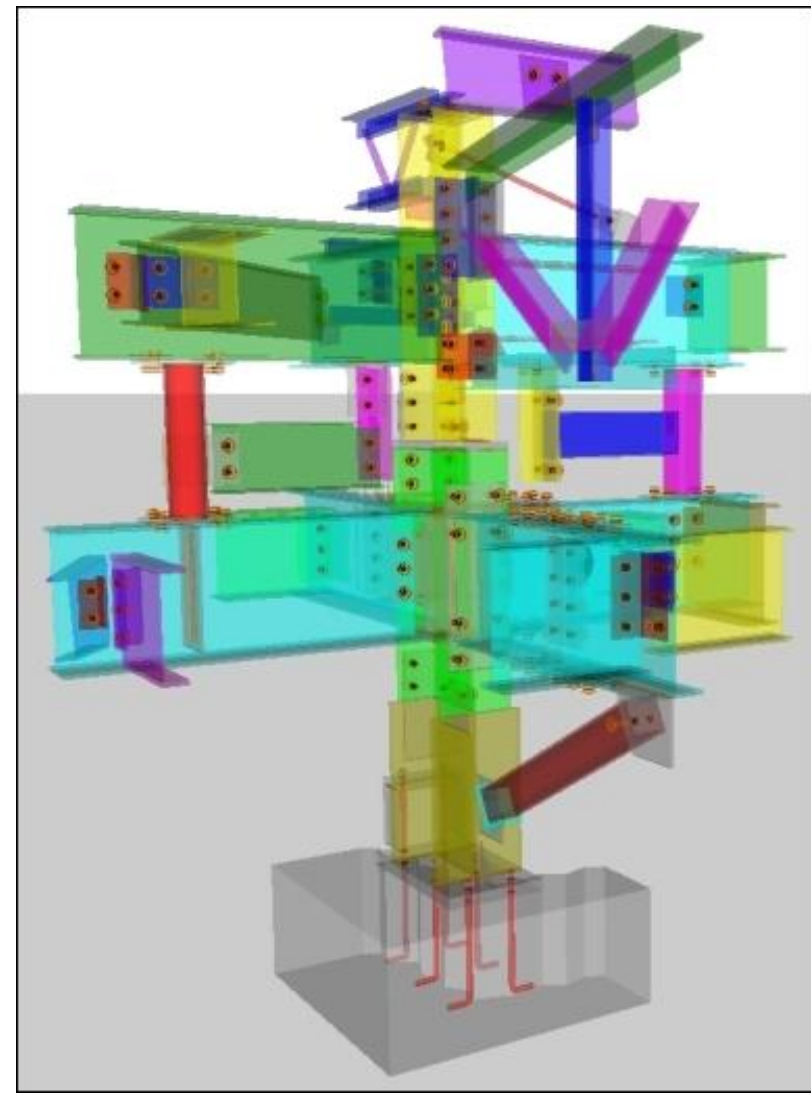

Figure 14: Transparent rendering

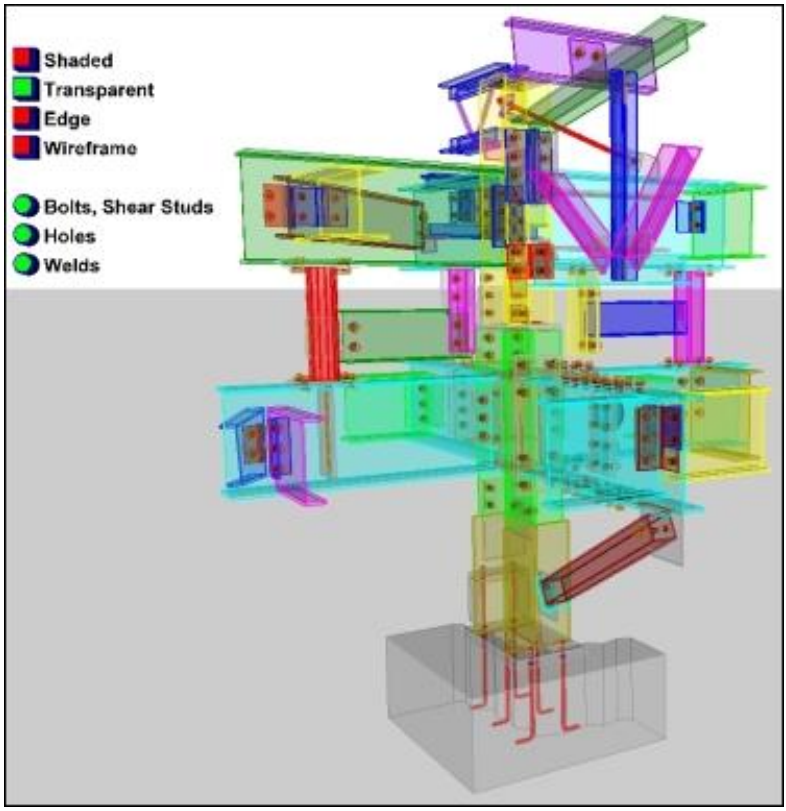

Figure 16: Rendering options in VRML model

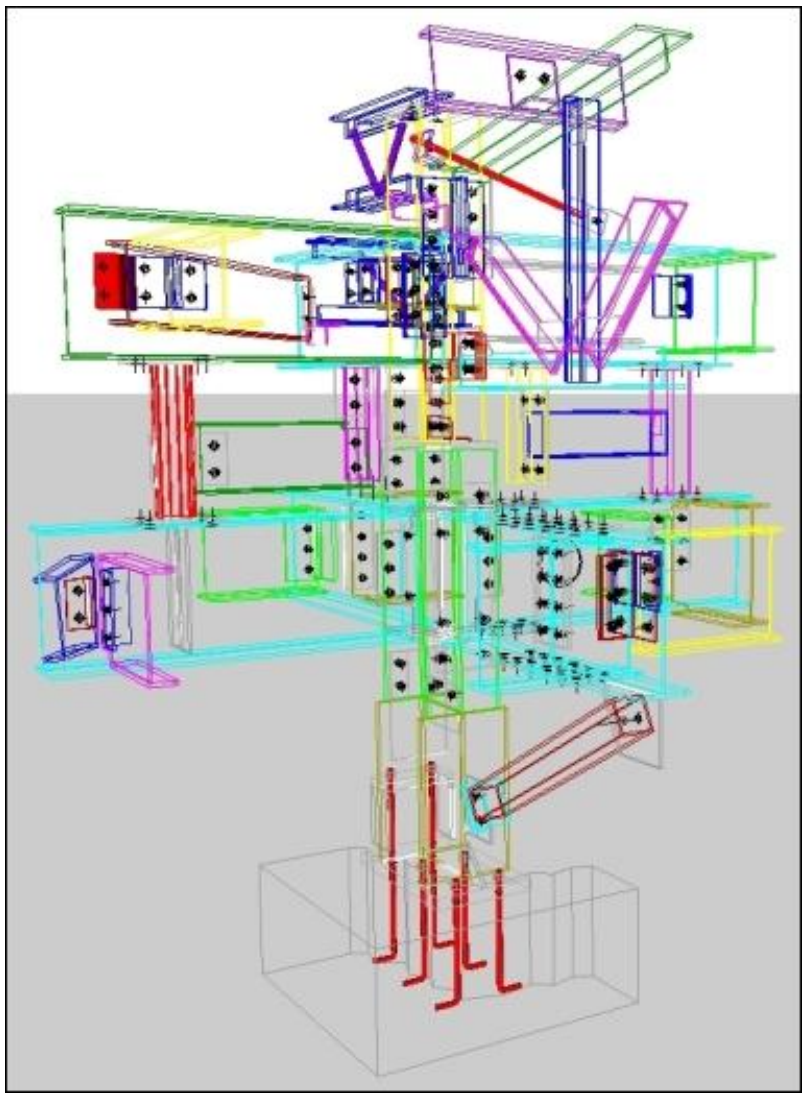

Figure 15: Edge rendering

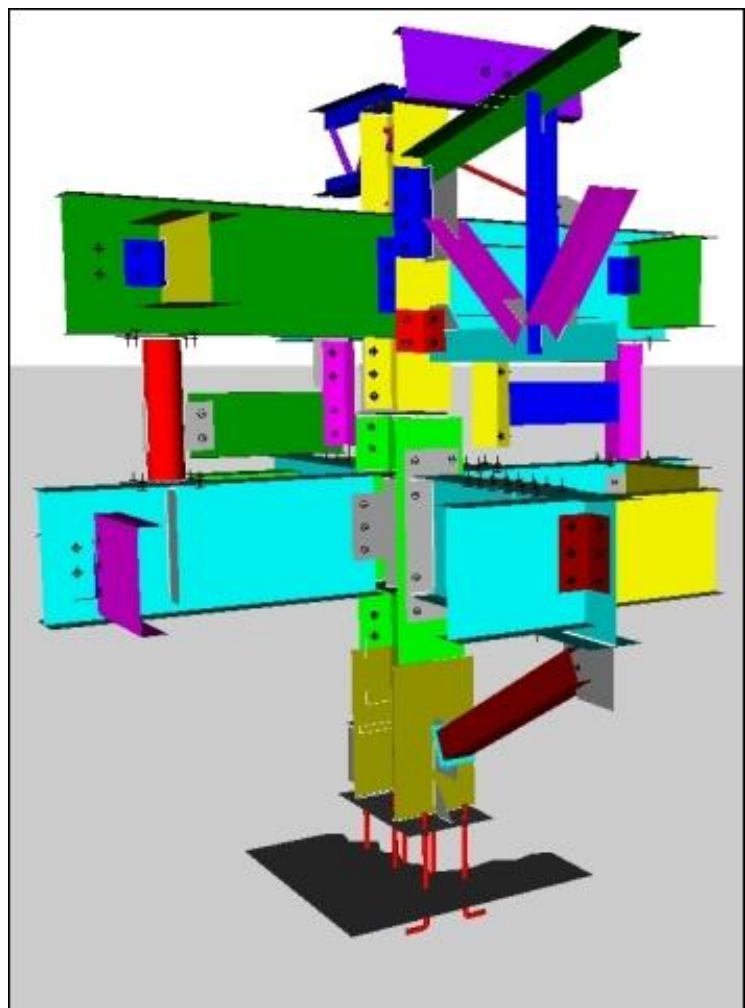

Figure 17: Ignoring thickness of members 


\subsection{Title, Ground Plane, Origin Axes}

Figure 18 shows the options to generate a title, ground plane, and origin axes in the VRML model.

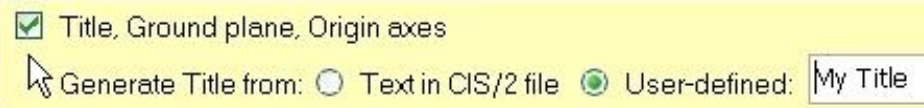

Figure 18: Title, ground plane, and origin axes options

Figure 19 shows a VRML model with a title, ground plane, and origin axes. The title in the VRML model is based on text information found in the CIS/2 file. If no text information is found in the CIS/2 file, a title is generated based on the type of CIS/2 model and the name of the software that created the CIS/2 file. A user-defined title can be specified to override the automatically generated title. The title might not be displayed in some VRML plugins and viewers.

The ground plane is shown as a rectangular outline at the base of the structure. The base of the structure is considered to be the lowest point in the structure. This is not necessarily the level of the "ground" in the structure.

The small origin axes (in blue) are visible at the base of the corner column on the left. The origin axes are displayed at the coordinates 0,0,0 in the VRML model which might not be near the actual structure and therefore not visible in the VRML model.

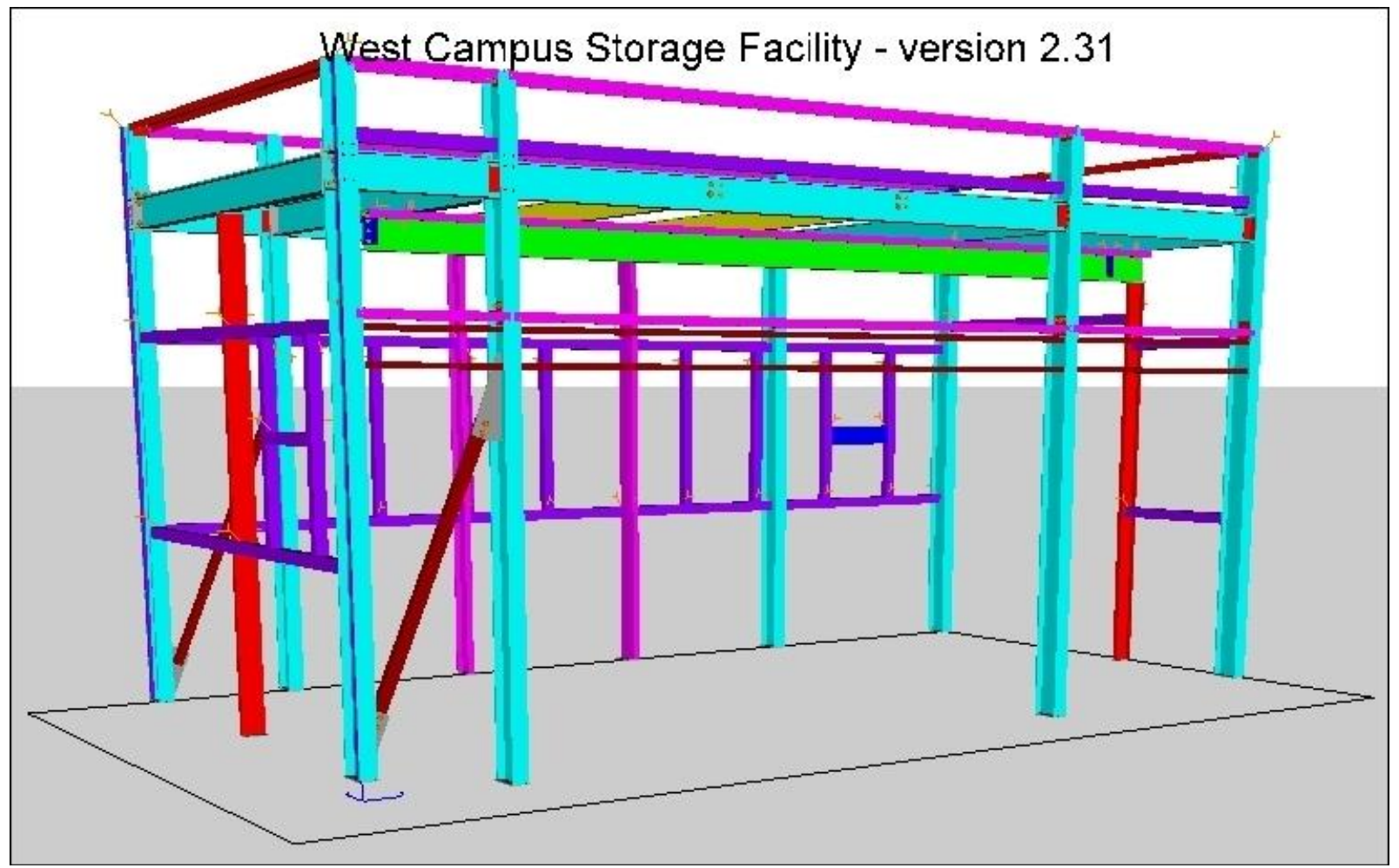

Figure 19: VRML model with title, ground plane, and origin axes 


\subsection{Viewpoints}

Figure 20 shows the options related to generating viewpoints in the VRML model. The viewpoints can be accessed in the VRML plugin or viewer that displays the VRML model. Typically, the PageUp and PageDown keys can be used to cycle through the viewpoints in the VRML plugin or viewer.

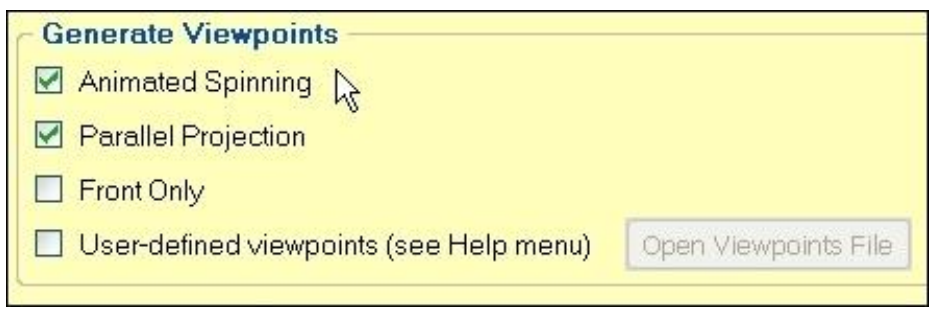

Figure 20: Generate viewpoint options

Thirteen default viewpoints corresponding to various Front, Side, and Top viewpoints are automatically generated. Optionally, an animated spinning and parallel (orthographic) projection viewpoints can be generated. Both types of optional viewpoints might not work in some VRML plugins and viewers. Using the Front Only option generates only one default viewpoint.

\subsubsection{User-Defined Viewpoints}

User-defined viewpoints are useful when sharing the VRML model to show another user where to look in the VRML model. It takes several manual steps to add user-defined viewpoints to the VRML model:

1. Select a CIS/2 file to translate. This will enable the User-defined viewpoints option.

2. Check the option for User-defined viewpoints. This will enable the "Open Viewpoints File" button.

3. Click the "Open Viewpoints File" button. This will open a file in a text editor. Text that will be displayed in the VRML model must be entered in the file to record the user-defined viewpoints.

4. Click the "Translate and Display" button. An example VRML model with the viewpoint text is shown in Figure 21. As the VRML model is rotated, the values for the position and orientation will update. For each user-defined viewpoint, the exact text from and including 'Viewpoint \{' to the ending ' $\}$ ' must be entered in the viewpoints file. In this example, the text is:

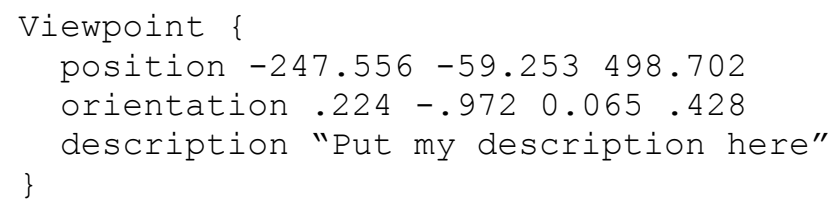

Any text can be used for the description. Clicking on the text "Click here to show viewpoint text" might open up a text window with the text that needs to be entered in the viewpoints file. If the text window appears, the viewpoint text can be copied from the text window and pasted into the viewpoints file.

5. Repeat the process of adding the text associated with the viewpoints to the viewpoints file for each user-defined viewpoint.

6. Save the viewpoints file and close it. Use the default name and location of the file.

7. Uncheck the option for User-defined viewpoints.

8. Click the "Translate and Display" button again. The saved viewpoints will be automatically included in the resulting VRML model every time the CIS/2 file is translated. The text for userdefined viewpoints will appear in the VRML plugin or viewer's menu of viewpoints. 


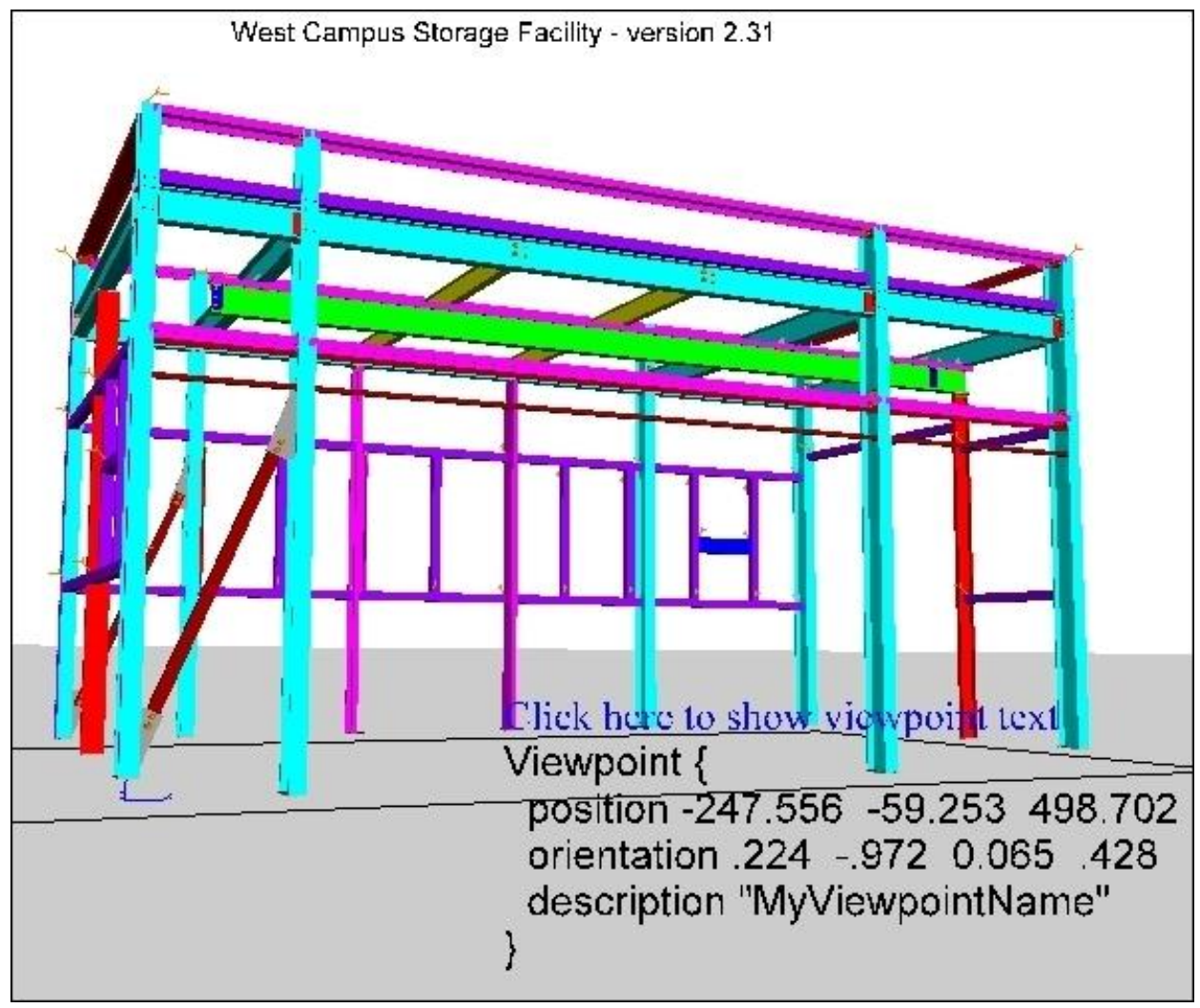

Figure 21: VRML model with viewpoint text 


\section{$5 \quad$ Text Reports}

Text reports include several ways to display text information related to the CIS/2 file in the VRML model or in a summary file. Most of the options related to text reports are in the Reports tab. The user interface for generating a summary report or text popups is shown in Figure 22.

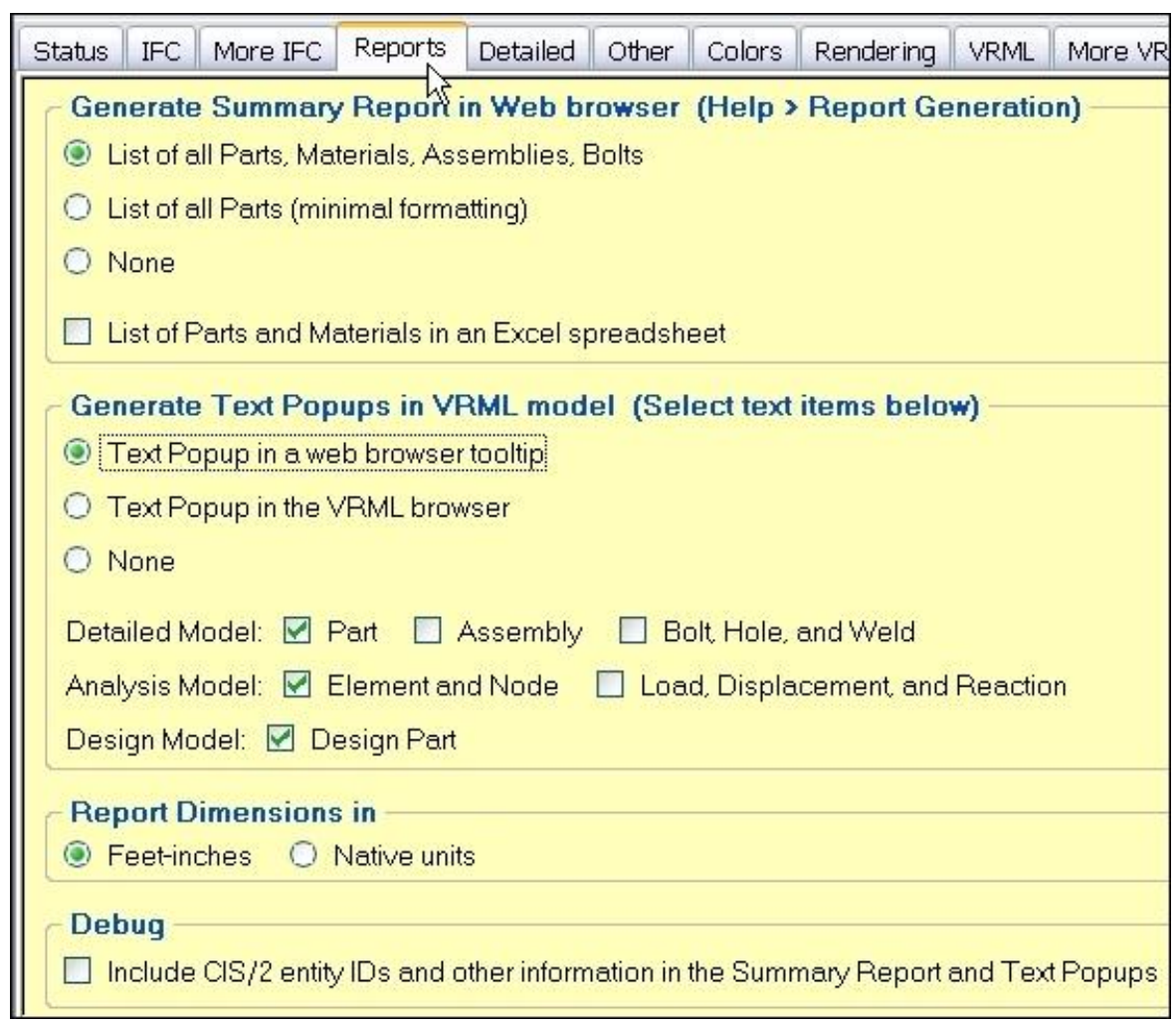

Figure 22: Reports tab options

\subsection{Summary Report}

The summary report is an HTML file that is displayed in the web browser and includes for all models, a list of all parts and materials. Information about assemblies and bolts are also included for detailed models. Figures 23 through 26 show portions of information that are available in the summary report. The summary report might also have information about the software used to generate the CIS/2 file, the user who generated the CIS/2 file, and other drawings associated with the model.

Options are also available to generate the summary report with minimal formatting or in an Excel spreadsheet. Either of these options might be useful to import the summary report into other applications.

If the CIS/2 file is named mycis $2 \mathrm{file.stp}$, then the resulting summary report file will be named mycis2file_sum.html. 


\subsubsection{Part List}

The headings for the summary report part list shown in Figure 23 are:

- Qty - quantity of the part

- Mark - piecemark of the part

0 design and analysis models usually do not have a piecemark and are listed as n/a

0 if the part is the main member of an assembly, then the piecemark text is bold

- $\quad$ Size - section designator of the part

- for plates that do not have a section designator, rectangular plates are listed as, for example, Plate (0-8x0-3) and non-rectangular plates are listed as Plate (Complex)

o the size is linked to the Material List

- Length - part length in feet and inches or the native units of the CIS/2 file depending on the selection in the Report tabs

$\circ$ if the length cannot be listed with fractions for inches, then decimal values are used

- the "length" of a plate is the thickness of the plate and is listed as $\mathrm{T}=3 / 4$

- Grade - material grade of the part

- Unit Wgt - unit weight of the part, see section 5.2 below for how weight is calculated

- Total Wgt - total weight of all the type of parts

- Function - the function depends on text information in the CIS/2 file

\begin{tabular}{|c|c|c|c|c|c|c|c|c|}
\hline \multicolumn{9}{|c|}{ Part List (Material List, Assembly List, Bolt List, $\underline{\text { People, }}$ Software) } \\
\hline & Qty & $\begin{array}{c}\text { Mark } \\
\text { (Bold-main) }\end{array}$ & \begin{tabular}{|c|} 
Size \\
(Link to Material List)
\end{tabular} & $\begin{array}{l}\text { Length } \\
(\mathrm{Ft}-\ln )\end{array}$ & Grade & $\begin{array}{l}\text { Unit } \\
\text { Wgt }^{*} \\
\text { (Lbs) }\end{array}$ & $\begin{array}{l}\text { Total } \\
\text { Wgt } \\
\text { (Lbs) }\end{array}$ & Function \\
\hline 1 & 14 & a1 & $\mathrm{L} 1 \times 1 \times 1 / 8$ & $91 / 2$ & A36 & 0.6 & 8.8 & Member \\
\hline 2 & 14 & a2 & L1 $1 \times 1 \times 1 / 8$ & $91 / 2$ & A36 & 0.6 & 8.8 & Member \\
\hline 3 & 1 & a3 & 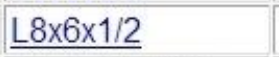 & $0-6$ & A36 & 12 & 12 & Column \\
\hline 4 & 4 & a4 & 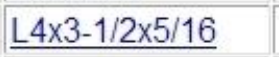 & $1-21 / 2$ & A36 & 9.2 & 37 & Beam \\
\hline 5 & 2 & a6 & $\underline{\mathrm{L} 3 \times 3 \times 1 / 4}$ & $19-43 / 16$ & A36 & 95 & 189 & Vertical brace \\
\hline 6 & 2 & a7 & $\underline{4} 4 \times 3 \times 5 / 8$ & $3-103 / 8$ & A36 & 53 & 105 & Beam \\
\hline 7 & 4 & a8 & $\mathrm{L} 3-1 / 2 \times 3 \times 5 / 16$ & $81 / 2$ & A36 & 4.7 & 19 & Horizontal brace \\
\hline 8 & 2 & a9 & 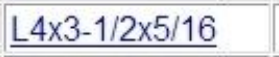 & $51 / 2$ & A36 & 3.5 & 7. & Beam \\
\hline 9 & 2 & a10 & 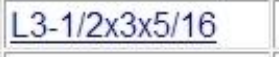 & $51 / 2$ & A36 & 3. & 6.1 & Beam \\
\hline
\end{tabular}

Figure 23: Summary report part list

Other information might be included in the part list and is dependent on the type of CIS/2 model and information written to the CIS/2 file, including:

- Holes per part - number and diameter of holes in the part

- Copes per part - number of type of copes (cutouts) per part

- Plate area and perimeter - area and perimeter length of the plate

- Orientation - in an analysis model, rotation angle of the part about its longitudinal axis

- Surface - surface treatment

- Part drawing, assembly drawings, CNC - links to drawings and CNC files

- Prelim Mark - preliminary marks

- Information related to advanced bills of material and fabrication status 


\subsubsection{Material List}

The headings for the summary report material list shown in Figure 24 are:

- Size, Grade - same as for the part list above

- Total Length - total length of all the parts of the same size

- Total Weight - total weight of all the parts of the same size, (section 5.2 below)

- Pieces - quantity of parts of a size

- Color - color of the part in the VRML model if the colors associated with the part size are unique

\begin{tabular}{|c|c|c|c|c|c|c|}
\hline \multicolumn{7}{|c|}{ Material List $(\underline{T o p})$} \\
\hline & Size & Grade & $\begin{array}{l}\text { Total Length } \\
\text { (Feet) }\end{array}$ & $\begin{array}{c}\text { Total Weight } \\
\text { (Lbs) }\end{array}$ & Pieces & Color \\
\hline 1 & C8x11.5 & $n / a$ & 120.9 & 1390 & 26 & Red \\
\hline 2 & C10x15.3 & $n / a$ & 259.89 & 3976 & 42 & Magenta \\
\hline 3 & DIA0.75 & $n / a$ & 507.06 & 761 & 73 & Purple \\
\hline 4 & DIA1.25 & n/a & 1099.65 & 4597 & 254 & 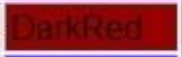 \\
\hline 5 & 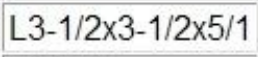 & $\mathrm{n} / \mathrm{a}$ & 144.04 & 1141 & 20 & \\
\hline 6 & W8x24 & n/a & 529.23 & 12702 & 40 & DarkGre \\
\hline 7 & W12x30 & $\mathrm{n} / \mathrm{a}$ & 1416.31 & 42489 & 85 & Cyan \\
\hline 8 & W12x35 & $n / a$ & 238. & 8330 & 14 & Green \\
\hline 9 & W12x53 & n/a & 80. & 4240 & 2 & DarkYellow \\
\hline 10 & W14x61 & $\mathrm{n} / \mathrm{a}$ & 312. & 19032 & 8 & Cyan \\
\hline 11 & W16x89 & $\mathrm{n} / \mathrm{a}$ & 449.78 & 40030 & 10 & Yellow \\
\hline 12 & WT6x13 & $\mathrm{n} / \mathrm{a}$ & 1086.5 & 14124 & 82 & Green \\
\hline 13 & WT $8 \times 22.5$ & $n / a$ & 101.3 & 2279 & 8 & Yellow \\
\hline Total & & & & & 664 & \\
\hline
\end{tabular}

Figure 24: Summary report material list 


\subsubsection{Assembly List}

The assembly list applies only to detailed models. The headings for the summary report assembly list shown in Figure 25 are:

- Qty - quantity of the assembly

- Mark - piecemark of the assembly

- Function - the function depends on text information in the CIS/2 file

- Unit Wgt - unit weight of the assembly, (section 5.2 below)

- Parts Per Assembly - piecemarks all the parts in the assembly

0 the number of parts is listed parenthetically before the part piecemark

$\circ$ the part piecemarks are linked to the Part List

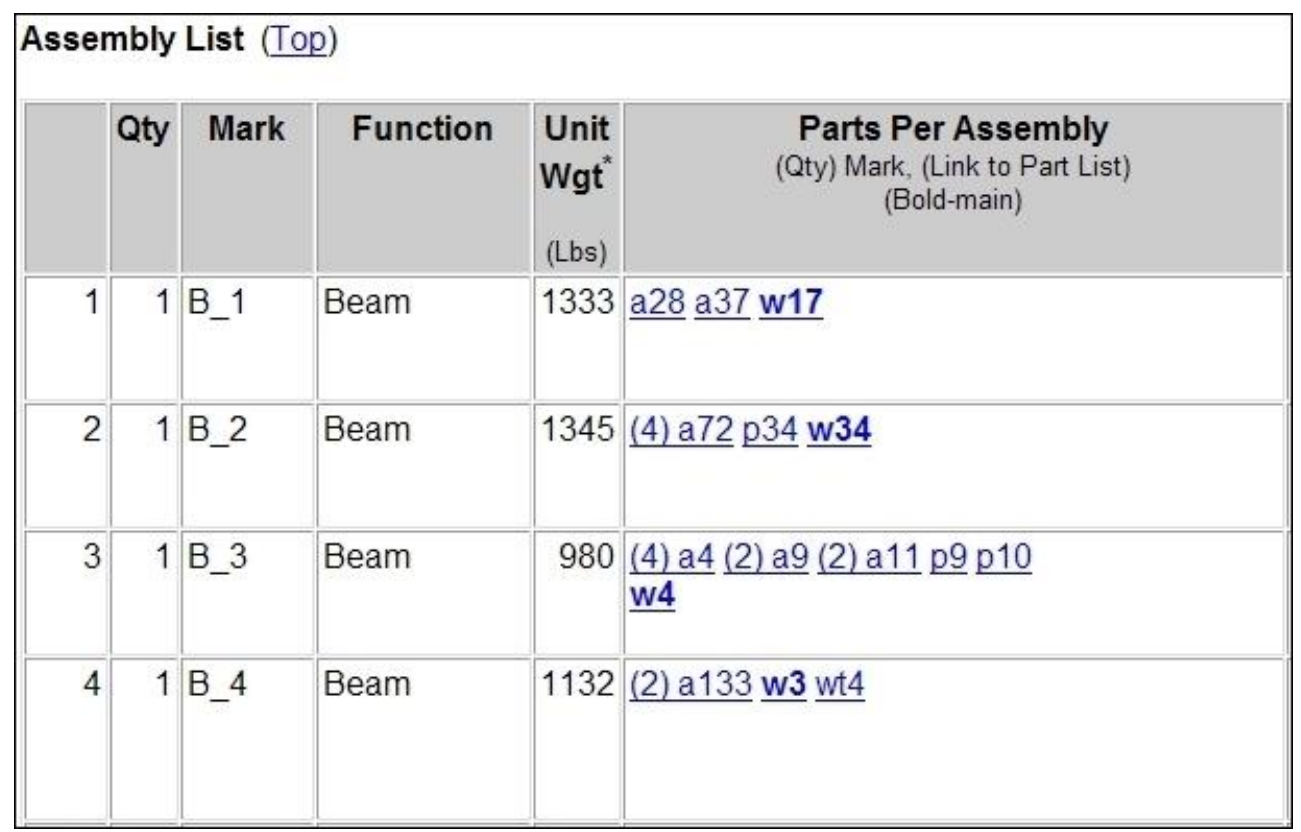

Figure 25: Summary report assembly list

Other information might be included in the assembly list and is dependent on the information written to the CIS/2 file, including:

- Bolts per assembly - number and size of bolts in the assembly

- Welds per assembly - number, type, and length (if available) of welds in the assembly

- Drawing - links to assembly drawings

- Information related to advanced bills of material and fabrication status 


\subsubsection{Bolt List}

The bolt list applies to detailed and design models. Shear studs are also included in the bolt list. The headings for the summary report bolt list shown in Figure 26 are:

- Qty - quantity of the bolt type

- Type - bolt diameter and length, Site or Shop, number and size of washers

\begin{tabular}{|r|r|l|}
\hline \multicolumn{3}{|l|}{ Bolt List (Top) } \\
\hline & Qty & \multicolumn{1}{|c|}{ Type } \\
\hline 1 & 16 & $\mathrm{D}=3 / 4 \mathrm{~L}=13 / 4$ Shop (1) $3 / 4$ (washer) \\
\hline 2 & 32 & $\mathrm{D}=3 / 4 \mathrm{~L}=13 / 4$ Site (1) $3 / 4$ (washer) \\
\hline 3 & 60 & $\mathrm{D}=3 / 4 \mathrm{~L}=2$ Site (1) 3/4(washer) \\
\hline 4 & 54 & $\mathrm{D}=3 / 4 \mathrm{~L}=21 / 2$ Site (1) $3 / 4$ (washer) \\
\hline 5 & 44 & $\mathrm{D}=3 / 4 \mathrm{~L}=21 / 4$ Site (1) $3 / 4$ (washer) \\
\hline 6 & 12 & $\mathrm{D}=3 / 4 \mathrm{~L}=23 / 4$ Site (1) $3 / 4$ (washer) \\
\hline Total & 218 & \\
\hline
\end{tabular}

Figure 26: Summary report bolt list

\subsection{Weight in Text Reports}

The weight of parts is not included in CIS/2 files. The weight reported in the summary report and text popups is computed by SteelVis and may not be accurate. The following cautions should be noted when considering the accuracy of the weight reported:

- Weight is computed from the part length and weight per length

- Weight computations might be affected by conversions between units for mass or units for length

- Weight is not computed for non-steel parts such as concrete, if they can be identified

- Weight for a part is not reduced by any material removed for copes, holes, and openings

- Weight per length for some sections comes from the lookup table (section 3.3.2 above)

- Sections not found in the lookup table will approximate the weight per length based on the cross section dimensions and density of steel

- Weight for plates is based on the actual volume of the plate regardless of its shape 


\subsection{Text Popups}

Text popups are short pieces of information related to parts, assemblies, bolts, welds, holes, analysis elements, loads, displacements, reactions, and design parts that appear (popup) in the VRML plugin, viewer, or the web browser status line when the mouse pointer is held over one of those items. The functionality of text popups is dependent on the VRML plugin, viewer, or the web browser. The options for text popups are found in the Reports tab as displayed in Figure 22 above. Two types of text popups are available; text popups in a tooltip and text popups in the VRML plugin or viewer.

\subsubsection{Text Popup in a Tooltip}

Figure 27 is an example of a text popup in a tooltip displayed with the Cortona3D VRML plugin. Figure 28 is an example of a text popup in a tooltip displayed with the Cosmo Player VRML plugin where the text appears in the status bar of the web browser. For web browsers that do not have a status bar such as Firefox, the tooltips for Cosmo Player cannot be displayed.

In these examples, five pieces of information are in the tooltip.

1. $\quad$ B_1 11$]-$ piecemark of the assembly that the part is associated with

2. $\mathrm{w} 4[12]$ - part piecemark

3. W12x22 - section designator

4. $\mathrm{L}=9-11 \frac{1 / 4}{4}$ length

5. $\mathrm{W}=219$ - weight

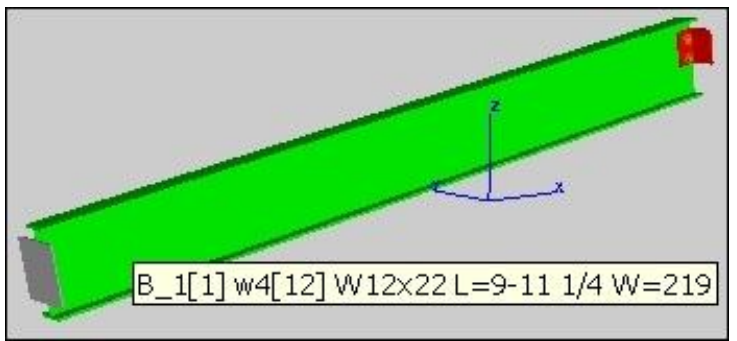

Figure 27: Tooltip text popup in Cortona3D

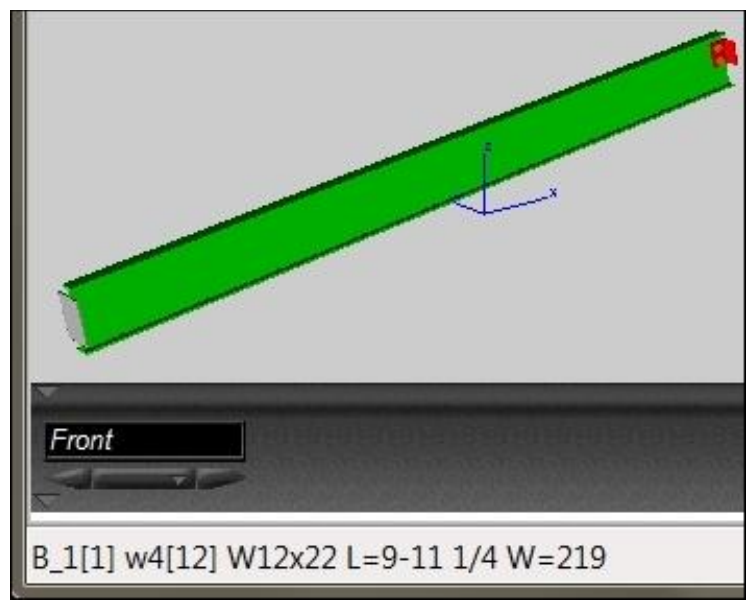

Figure 28: Tooltip text popup in Cosmo Player

Other information might be included in the tooltip and is dependent on the information written to the CIS/2 file. The information is similar to the information for the same item that is written to the summary report.

Sometimes the amount of the information is too long to be easily displayed in the tooltip. In this case, the text will begin with "Click for more:". Clicking on the item will open a web browser window with the complete text information. This feature only works when web browser popups are allowed or the web browser popup blocker is disabled. Figure 29 shows the tooltip text popup with assembly information displayed with the "Click for more:" feature. The text lists the four parts and bolts in the assembly. This type of text popup is the only way information about loads and reactions can be displayed for analysis models, however, it does not work in Internet Explorer. 


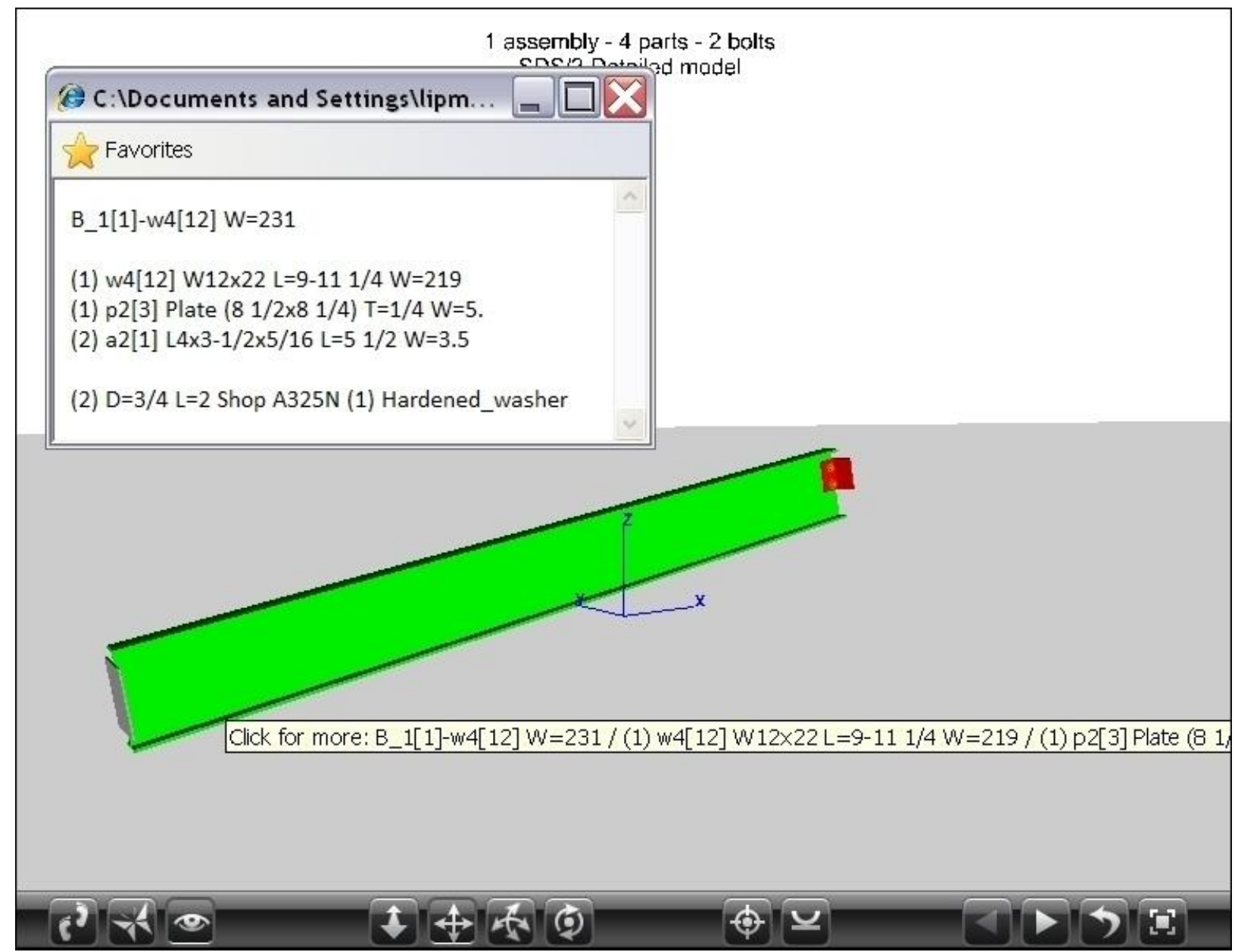

Figure 29: Tooltip text popup with assembly information in a separate web browser window 


\subsubsection{Text Popup in the VRML Browser}

An example of the text popup in the VRML browser is shown in Figure 30. The text information is for the entire assembly (similar to Figure 29) and overlays the VRML display. This method of displaying the text popup in the VRML browser depends in the VRML plugin or viewer. This option is not recommended for very large CIS/2 files.

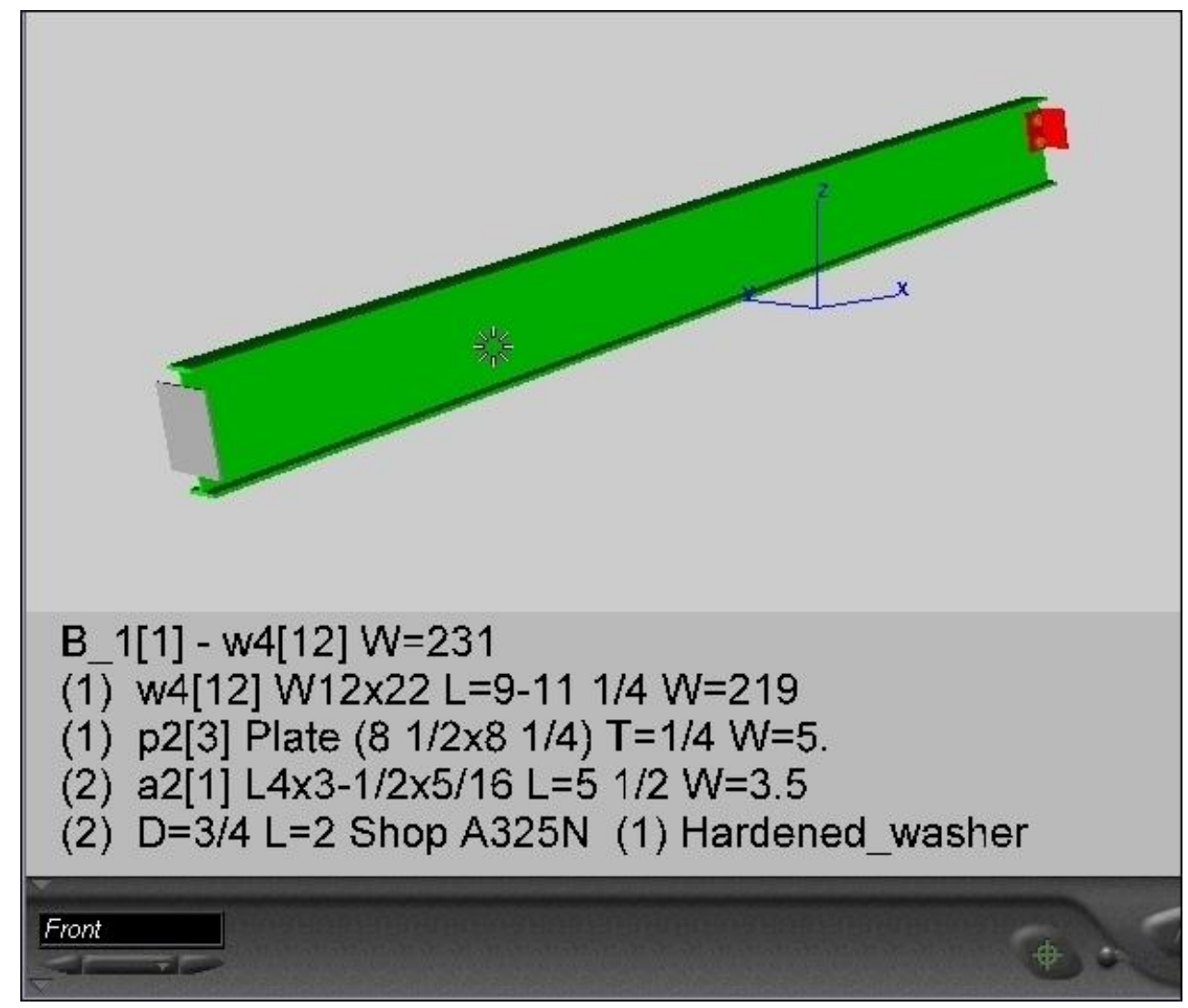

Figure 30: Text popup in the VRML browser 


\subsection{Member Labels}

The option for "Piecemark label for each member" can be selected in the Other tab. An example of the labels is shown in Figure 31. The labels usually contain the part piecemark and the section designator. The label is scaled for the length and depth of the member and is located at its mid-length. In the VRML browser, the labels rotate so that they always face forward and labels that are too small to be legible are not displayed.

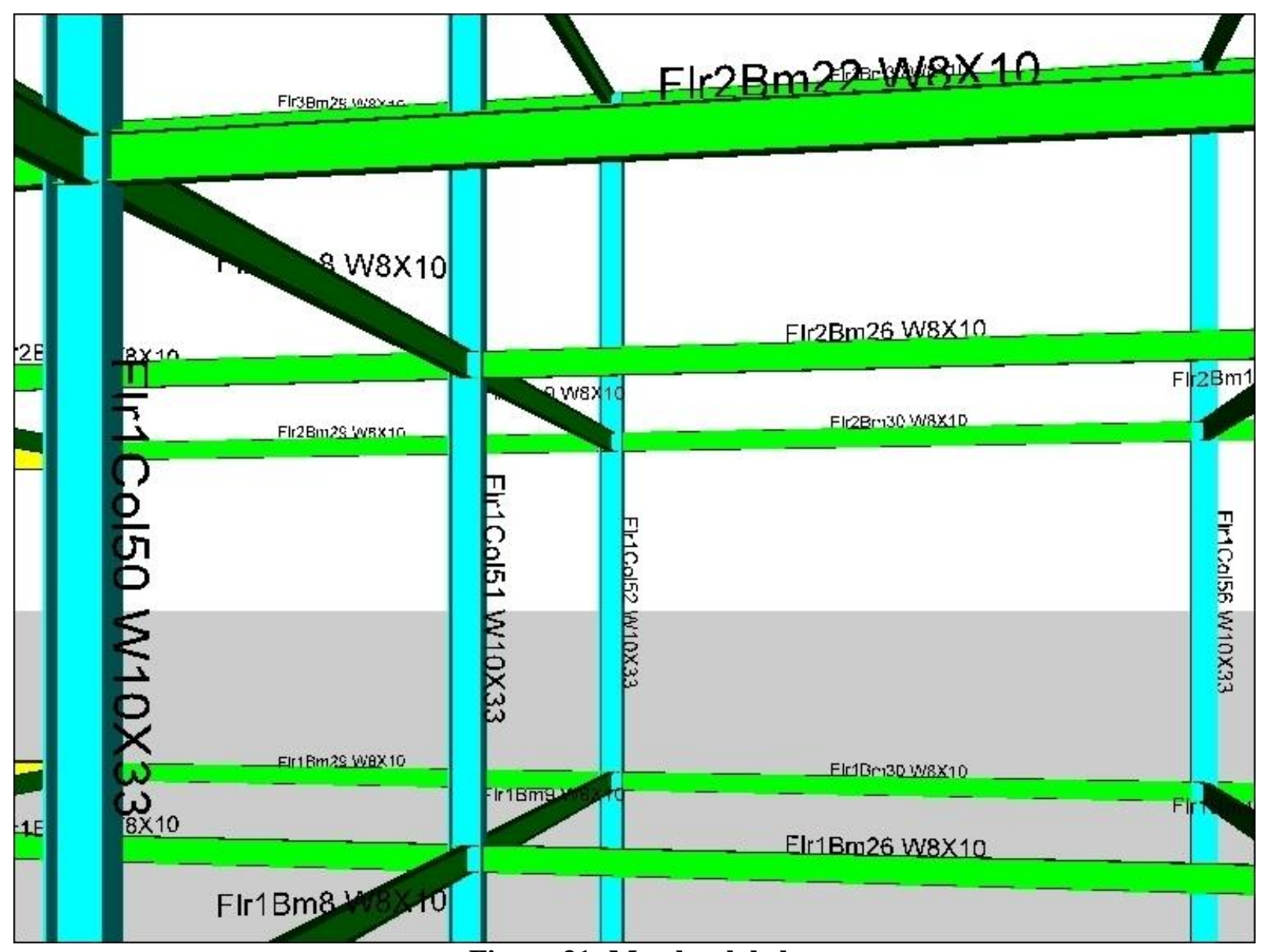

Figure 31: Member labels 


\section{Model Specific Features}

\subsection{Detailed Model}

The options related to detailed models are in the Detailed tab and are shown in Figure 32.

\begin{tabular}{|l|l|}
\hline Status IFC More IFC Reports Detailed Other Colors Rende \\
$\square$ Copes, Camber, Taper \\
$\square$ Bolts, Shear Studs \\
$\square$ Holes, Openings \\
$\square$ Welds \\
$\square$ Sequences, Zones \\
$\square$ Do NOT translate (exclude) Non-Main Parts in an assembly \\
$\square$ Do NOT translate (exclude) any Plates in the model \\
For each Assembly, generate in the VRML model \\
$\square$ Viewpoint \\
$\square$ On-Off switch \\
Debug \\
$\square$ Include comments about parts and connections in the VRML file
\end{tabular}

Figure 32: Detailed model options

\subsubsection{Copes, Camber}

The types of copes (cutouts) that are translated are: notches, chamfers, flange notches, flange chamfers, miter cuts, and cutting planes. Other types of copes that can be modeled in CIS/2 are not supported by SteelVis. Copes are not displayed on curved parts, plates, decking, and parts with a zee cross section. Some copes are not displayed on parts with a circular or rectangular cross section. Complex combinations of copes might not be displayed correctly in the VRML model.

If camber is included in the CIS/2 file, an approximation for its appearance is used in the VRML model. Cutting planes can be toggled on and off in the VRML model but they do not remove material from the parts they are associated with.

In Figure 33, the dark blue channel to the left of the I-beam has a miter cut on the right end and the magenta channel to the right of the I-beam has a notch on the bottom left end. 


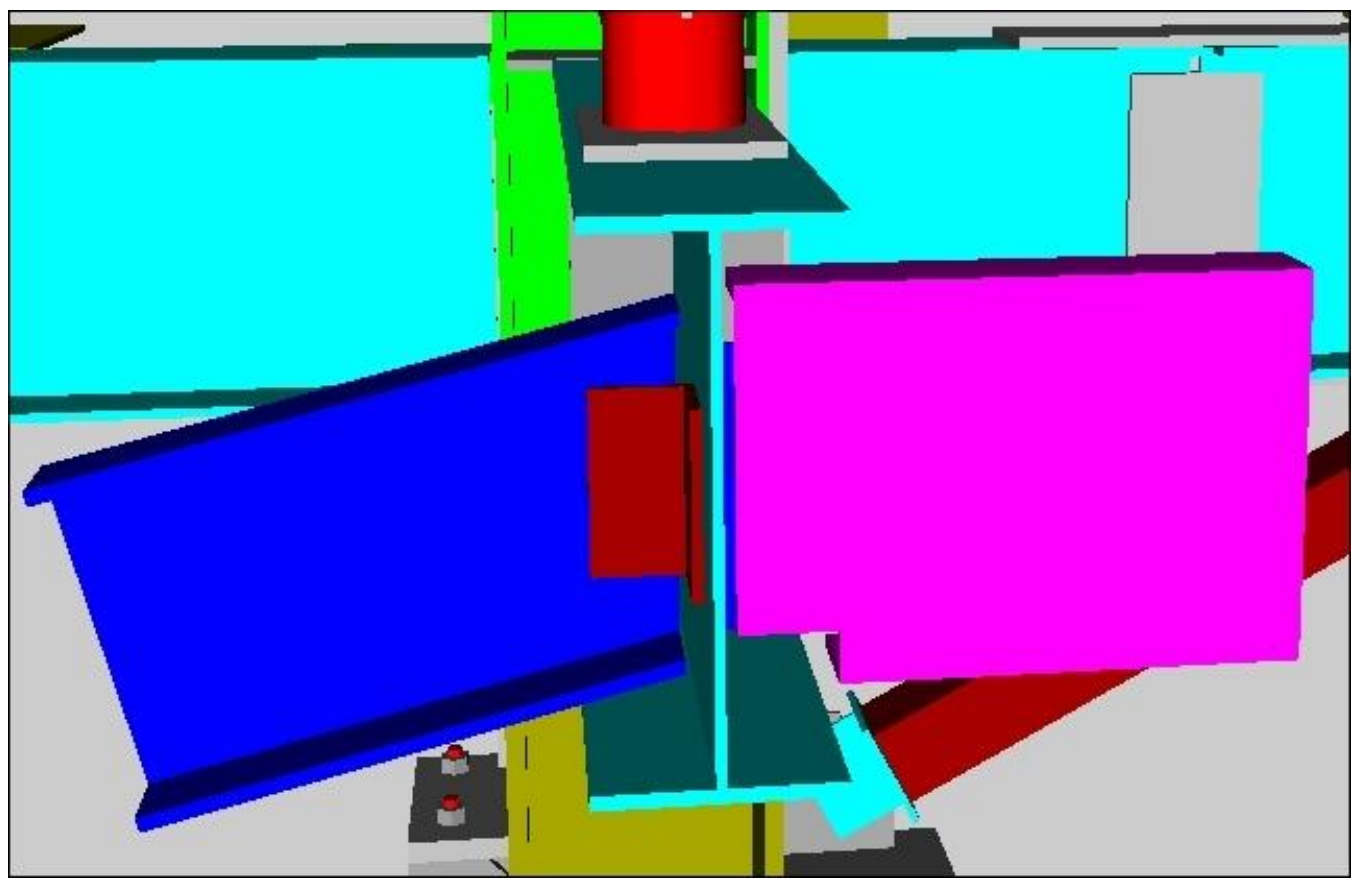

Figure 33: Parts with copes (cutouts)

\subsubsection{Bolts, Holes, and Welds}

Figure 34 shows several connections with bolts, holes, welds, and a shear stud. Figure 35 shows the same connections without the bolts and shear stud. Bolts always have a hexagonal head. If nuts and washers are included in the CIS/2 file, they are represented by hexagonal and circular rings, respectively, around the shaft of a bolt. The size and position of the rings do not reflect the actual size and position of the nuts and washers. If two circular rings are displayed, then two washers are used with the bolt, however, the second washer is usually located under the head of the bolt. A beige colored shear stud is visible in the lower right of Figure 34.

If weld paths are included in the CIS/2 file, they are represented by an orange fillet between the parts that are welded together. The cross section of the weld does not reflect the actual size, type, and geometry of the weld. The orange weld fillet might appear distorted if the weld path has sharp corners. If welds without weld paths are included in the CIS/2 file, their location is represented by an orange weld symbol as shown in Figure 36.

Holes and openings are represented by a black surface patch on the part through which the hole is drilled or the opening is cut or formed as seen in Figure 35. Holes can be circular, slotted, or rectangular. Openings, such as web penerations, can be any arbitrary shape. In some CIS/2 files, holes with a zero radius are used to represent a specific location on the part and are represented by an "X" in the VRML model. Holes and openings are not displayed on curved parts, bent plates, decking, and parts with a circular cross section.

Design models can also have shear studs, holes, and openings. 


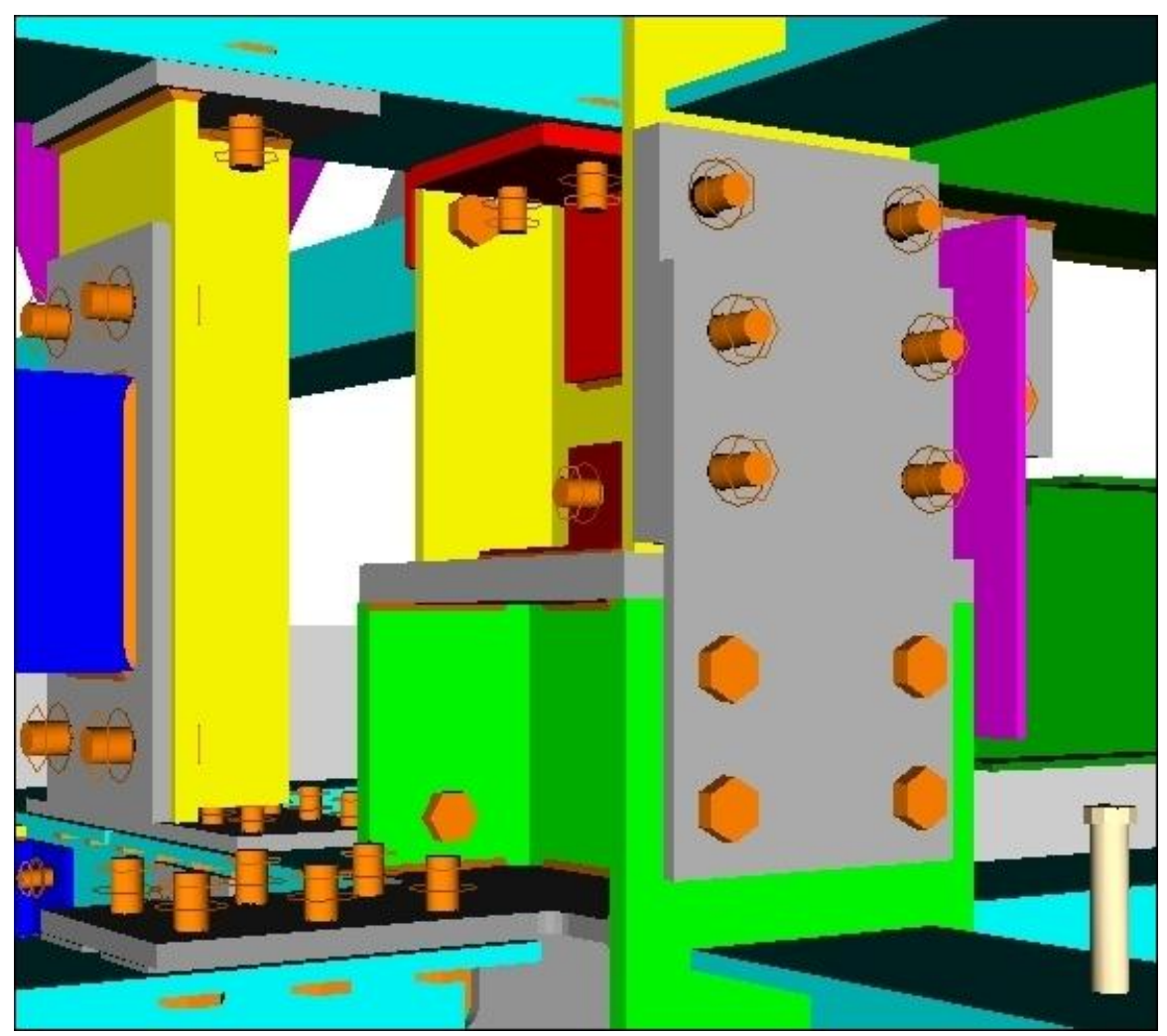

Figure 34: Parts with bolts, nuts, washers, holes, welds, shear stud

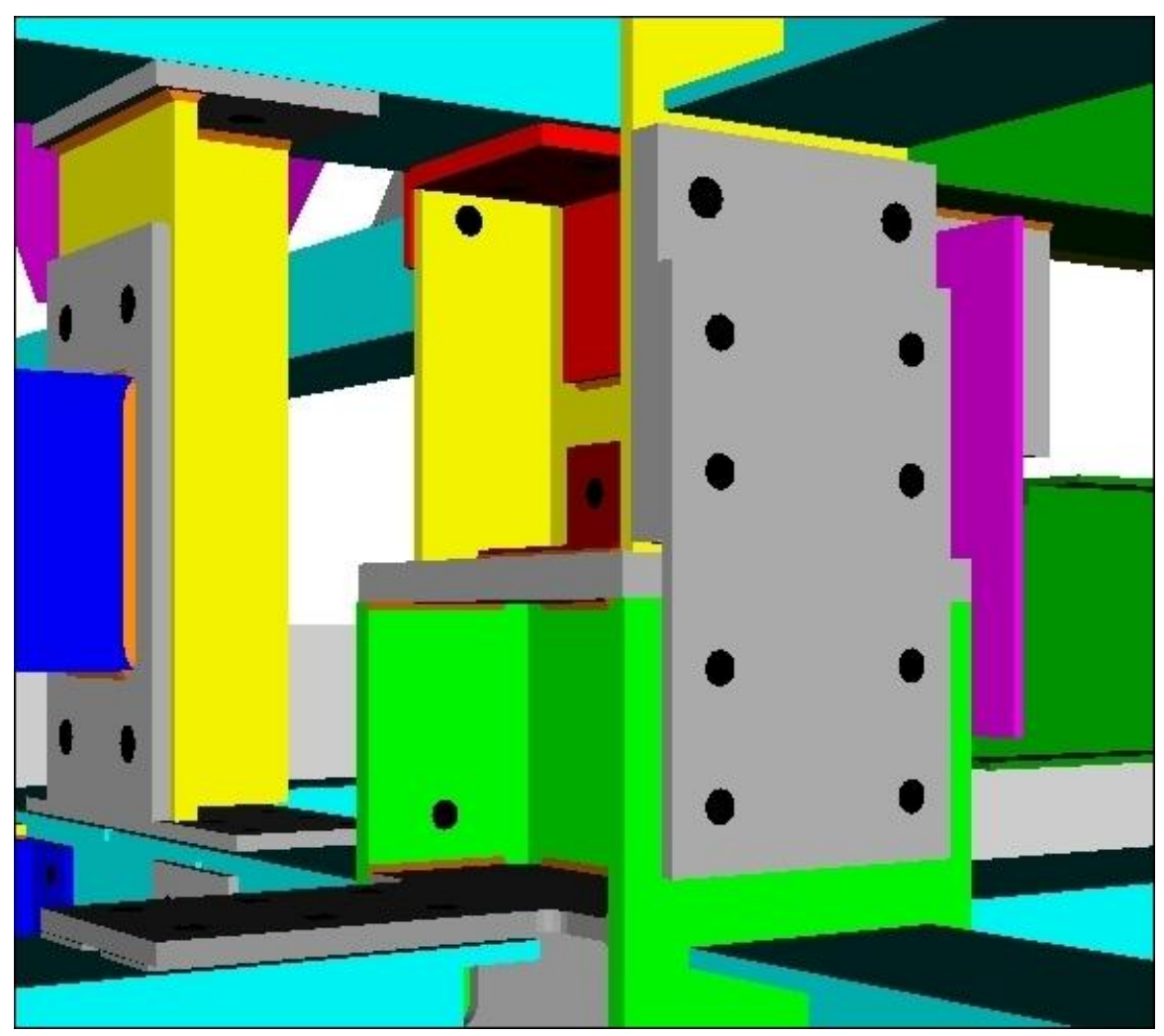

Figure 35: Parts with holes and welds 


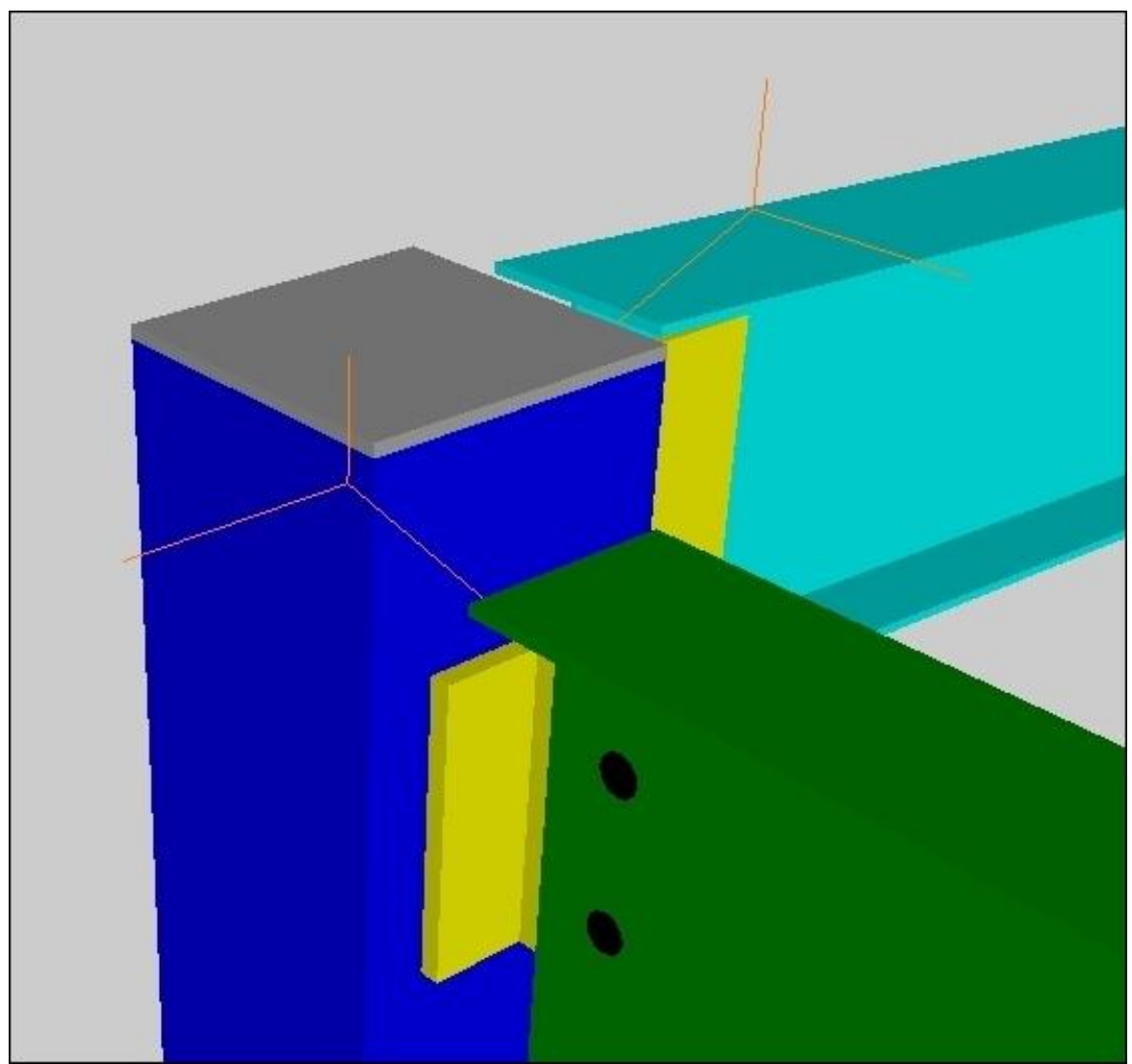

Figure 36: Welds represented by an orange weld symbol 


\subsubsection{Sequences}

If sequences are included in the CIS/2 file and sequences option is selected in the Detailed tab, then it is possible to toggle on and off the individual sequences in the VRML model. Figure 37 shows a structure with five sequences. Each sequence has a small round button associated with it to toggle on and off the parts in that sequence. In this example, the decking in sequence 4 is toggled off. Color is assigned based on groupings of members by sequences (section 4.1 above). All members in the same sequence have the same color. If sequences are written to the CIS/2 file, they do not necessarily represent an actual construction sequence.

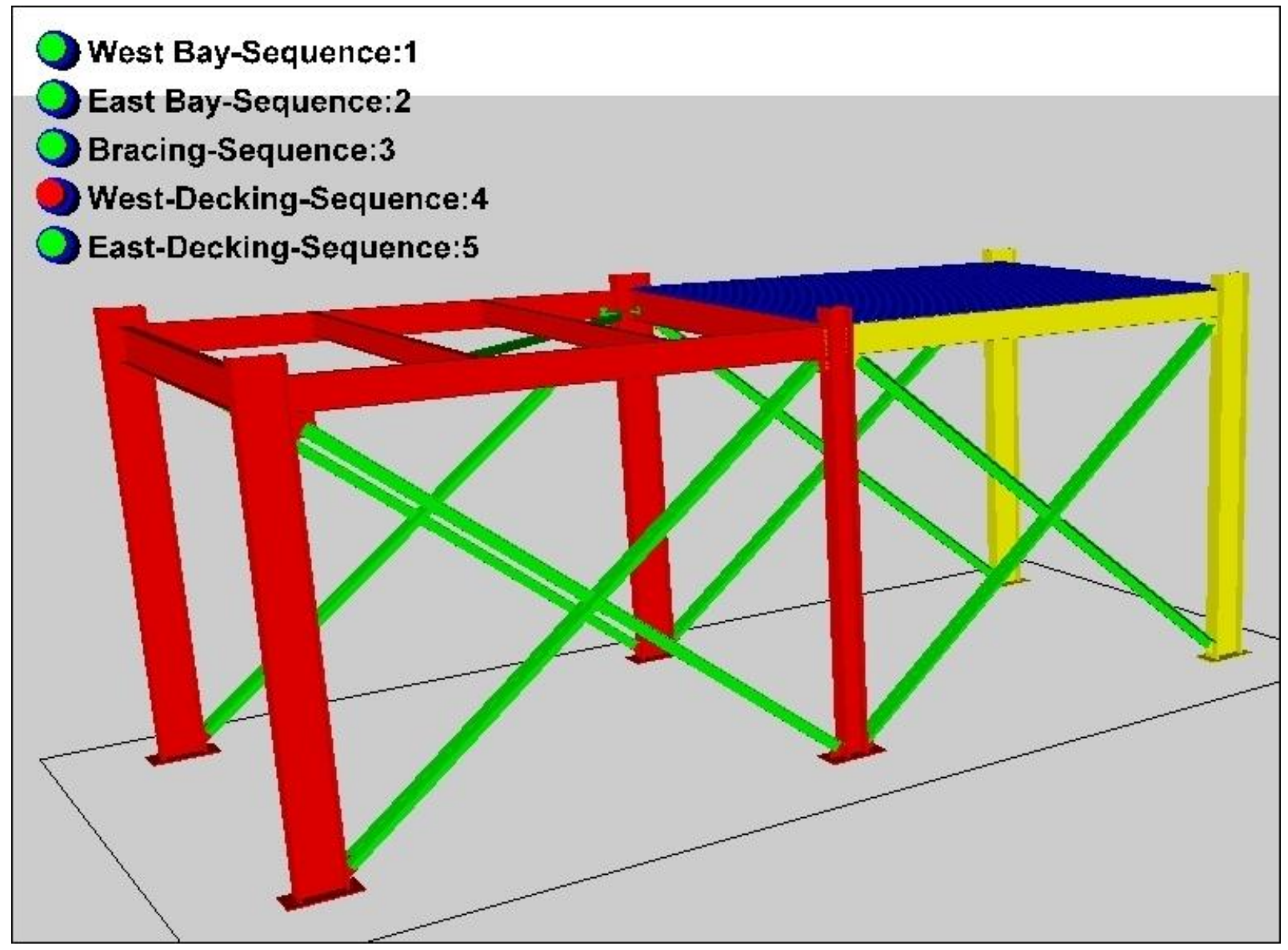

Figure 37: Structure with sequences 


\subsubsection{Excluding Parts}

In a detailed model, it is possible to exclude some types of members from the resulting VRML model. The options to exclude parts are the two options titled "Do NOT translate ..." in the Detailed tab as shown in Figure 32 above. This is useful when displaying very large CIS/2 files.

The types of members that can be excluded are non-main parts in an assembly and any plates. CIS/2 files with main members use the entity Located_part_marked and can be checked for in the Status tab in the "Translated CIS/2 entities" section. Non-main parts in an assembly are typically angle brackets and gusset plates. Figure 38 shows a structure with the non-main parts and plates excluded.

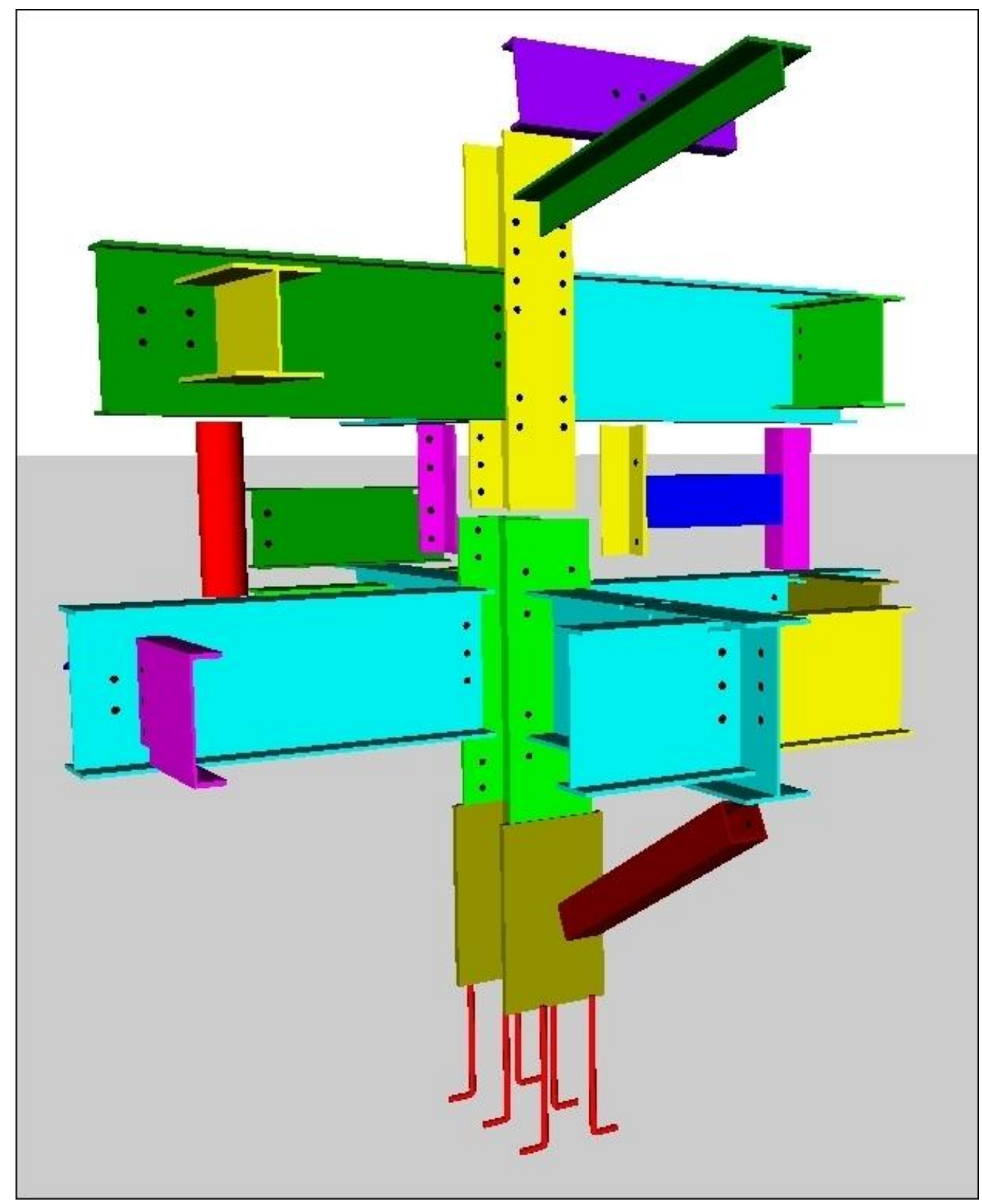

Figure 38: Structure with non-main parts and plates excluded 


\subsubsection{Assemblies}

Options are available to generate assembly viewpoints and assembly on-off switches in the VRML model. Assembly viewpoints are added to the default set of viewpoints (section 4.4 above). The viewpoint will position and orient the VRML model near the origin of the assembly.

Figure 39 shows a structure with assembly on-off switches. The switches appear as a red or green transparent cube in the VRML model. The text in the center of the cube is the assembly piecemark. Clicking on a red cube will hide the assembly it is associated with and the cube will turn green. Clicking on a green cube will make assembly visible again. In this example assembly "2-2" is hidden. This is a useful method to determine which parts and bolts are and are not associated with an assembly.

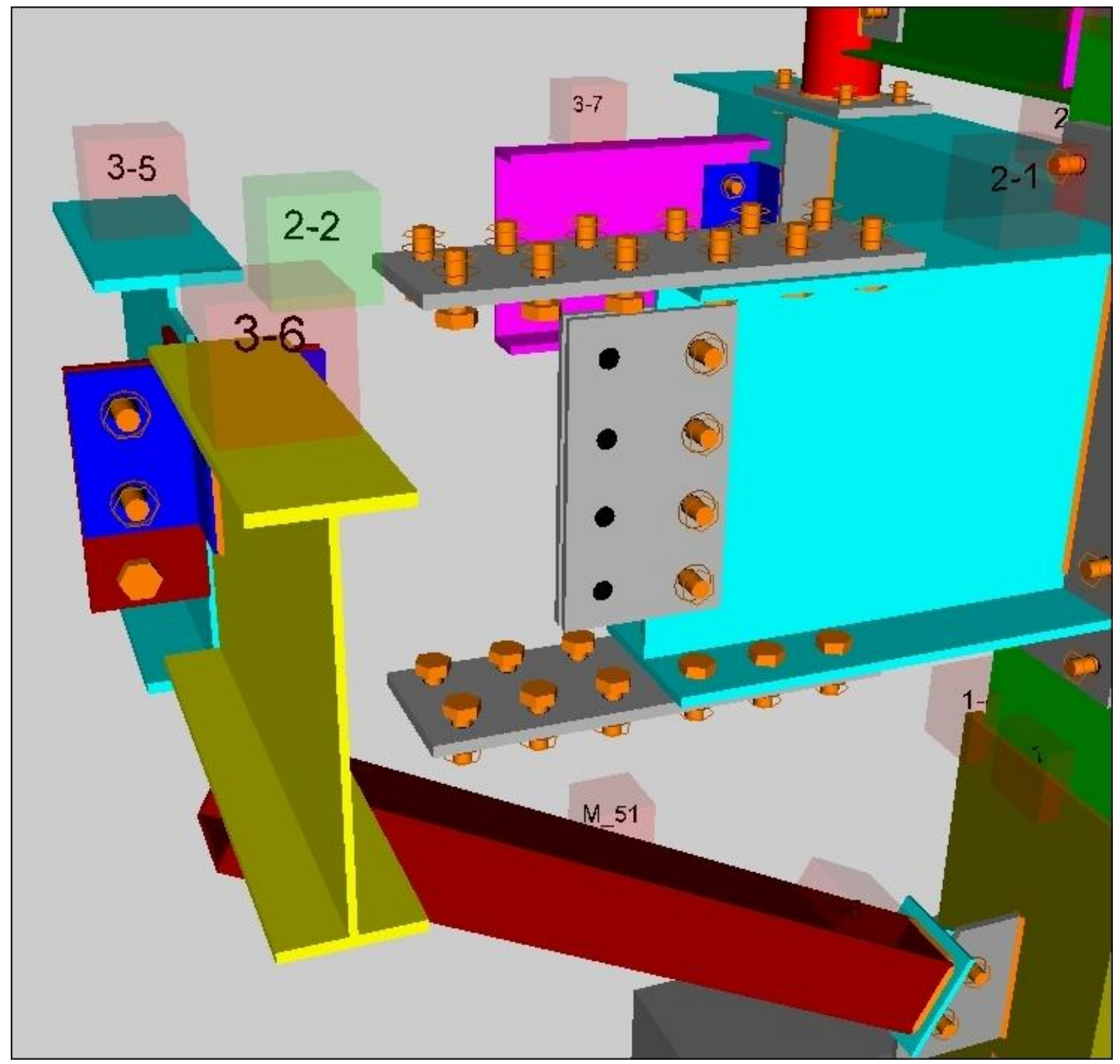

Figure 39: Structure with assembly on-off switches 


\subsection{Analysis Model}

\subsubsection{Nodes and Wireframe}

The options for analysis models are in the Other tab and are shown in Figure 40. The VRML model for an analysis model will always include the physical representation of the analysis elements. If the wireframe and nodes options are selected, then the wireframe analysis model and nodes are displayed as shown in Figure 41. Nodes are represented in the VRML model as a small transparent orange cube. VRML buttons can be used display to toggle on and off the physical representation of the analysis elements, the wireframe analysis model, and the nodes. In this example the analysis model elements are turned off. The wireframe model does not account for any analysis element eccentricity.

Analysis model loads, displacements, and reactions are not displayed in the VRML model. The only option for any feedback about loads, displacements, and reactions is with a text popup (section 5.3.1 above).

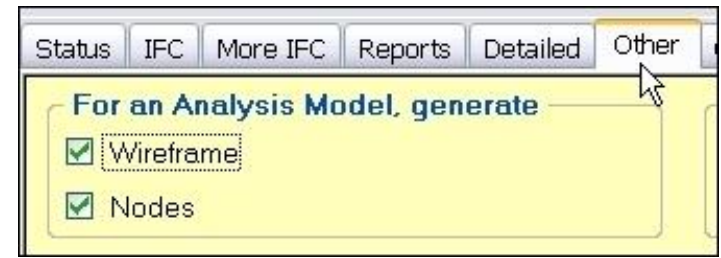

Figure 40: Analysis model options

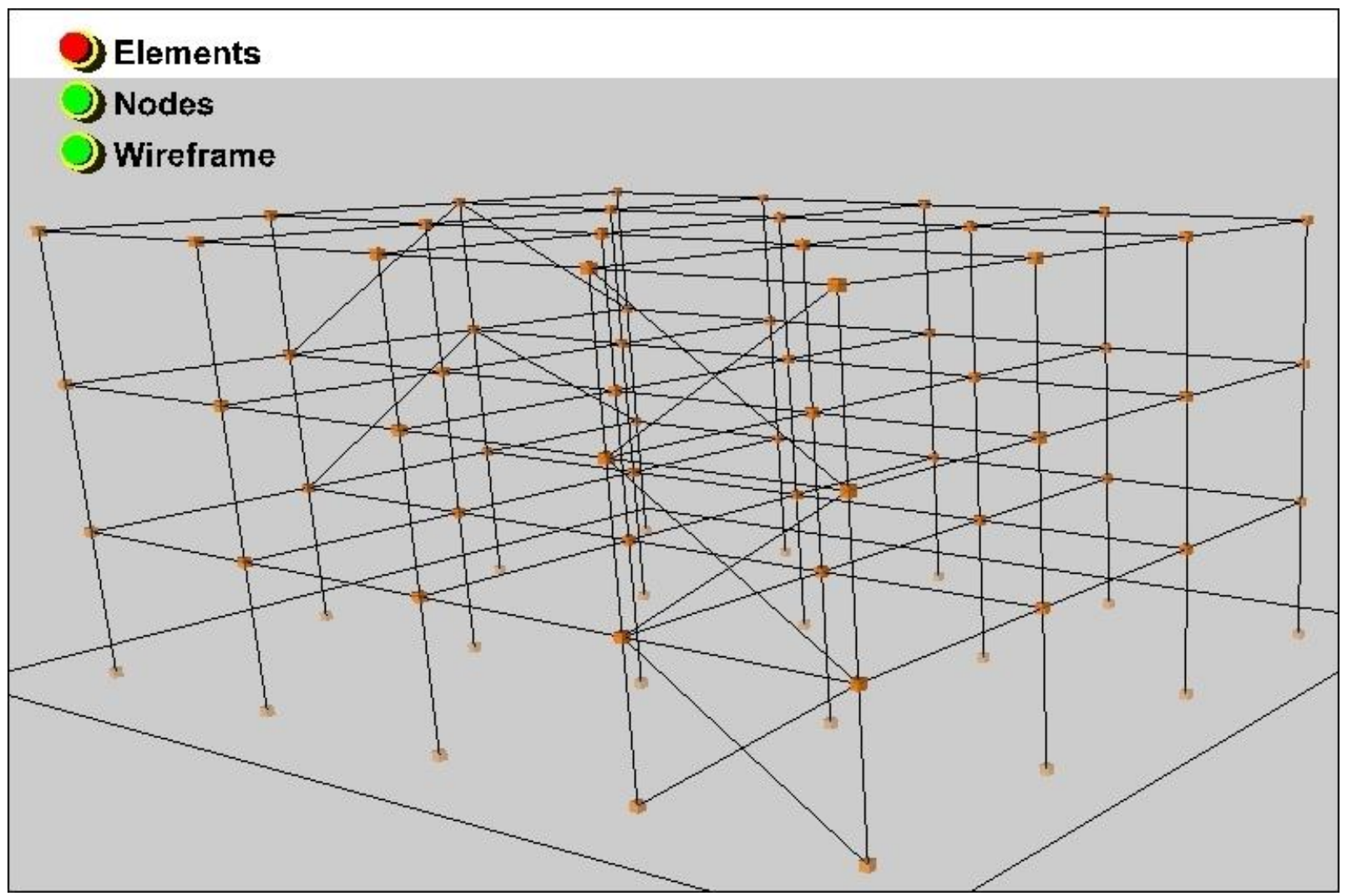

Figure 41: Analysis model wireframe and nodes 


\subsection{Design Model}

The options for design models are in the Other tab and are shown in Figure 42. Design connections and design joints systems as described below, however, they are not often included in CIS/2 files. Some design models also contain shear studs, holes, and openings. Options for those features are in the Detailed tab and are described in section 6.1.2 above.

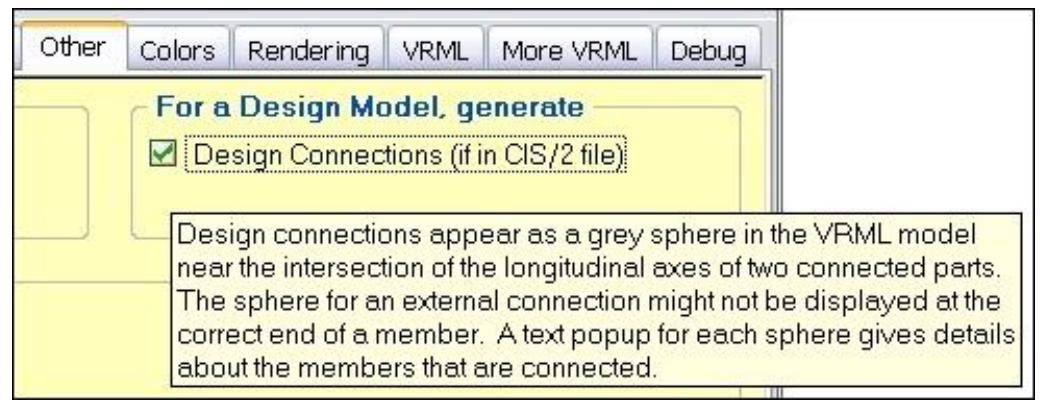

Figure 42: Design model options with tooltip help

\subsubsection{Design Connections}

A design connection indicates that a structural connection, such as a beam to column or beam to beam connection is to be made between several structural members. Design connections appear in the VRML model as a transparent grey sphere located near the intersection of the longitudinal axes of the connected parts but not necessarily where the parts are actually connected. The sphere for an external connection might not be displayed at the correct end of a member.

Figure 43 shows internal design connections between several members. If text popups are enabled for design parts (section 5.3.1 above), then information about the design connection will be associated with the grey sphere. In this example, the design connection is an internal connection that connects four parts, M341, M377, M394, and M52. CIS/2 files with design connections use the entity

Assembly_design_structural_connection_internal and can be checked for in the Status tab in the "Translated CIS/2 entities" section.

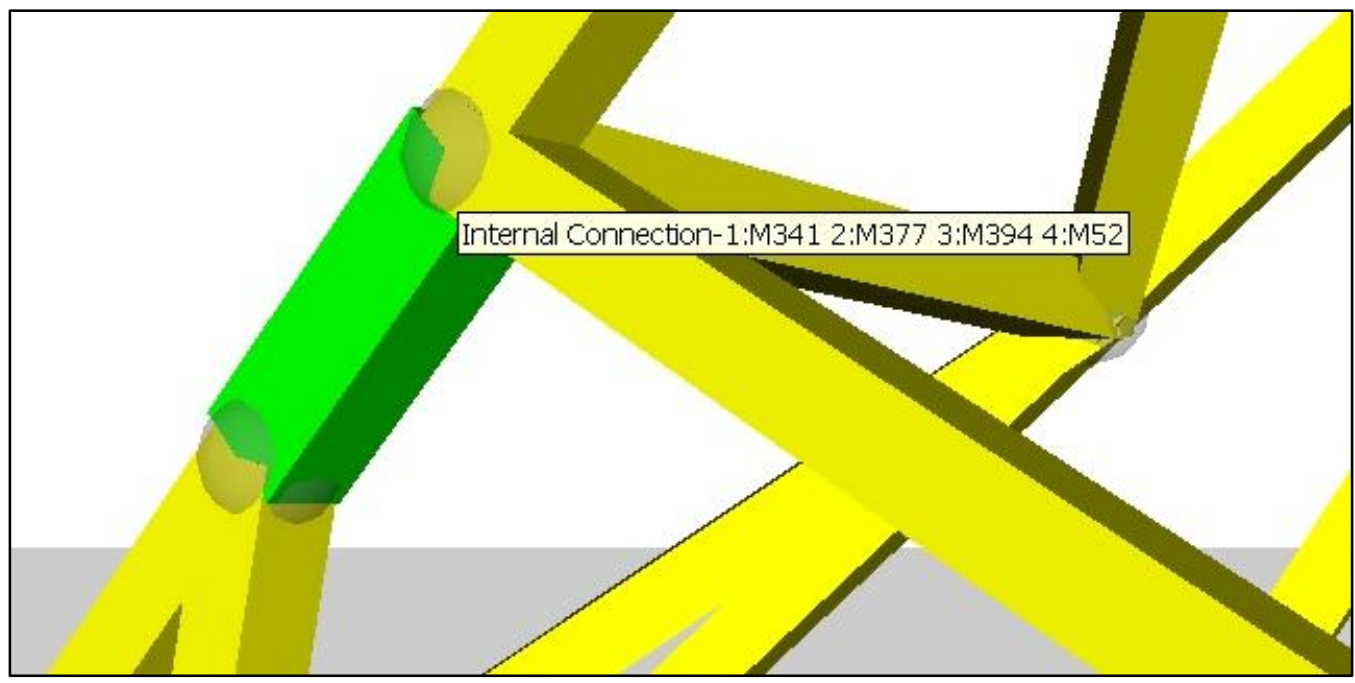

Figure 43: Internal design connection between four parts 


\subsubsection{Design Joint System}

A design joint system is a conceptual device for connection design. It is a representation of what type and materials should be used for a connection design without actually modeling the exact details of the connection. Design joint systems appear in the VRML model as an inverted transparent grey pyramid where the physical connection should be located. Figure 44 shows design joint system at the end of a beam. If text popups are enabled for design parts (section 5.3.1 above), then information about the design joint system will be associated with the grey pyramid. In this example, the design joint system indicates that the connection at the end of beam B_11 should use bolted double clip angles. CIS/2 files with design joint systems use the entity Design_joint_system and can be checked for in the Status tab in the "Translated CIS/2 entities" section.

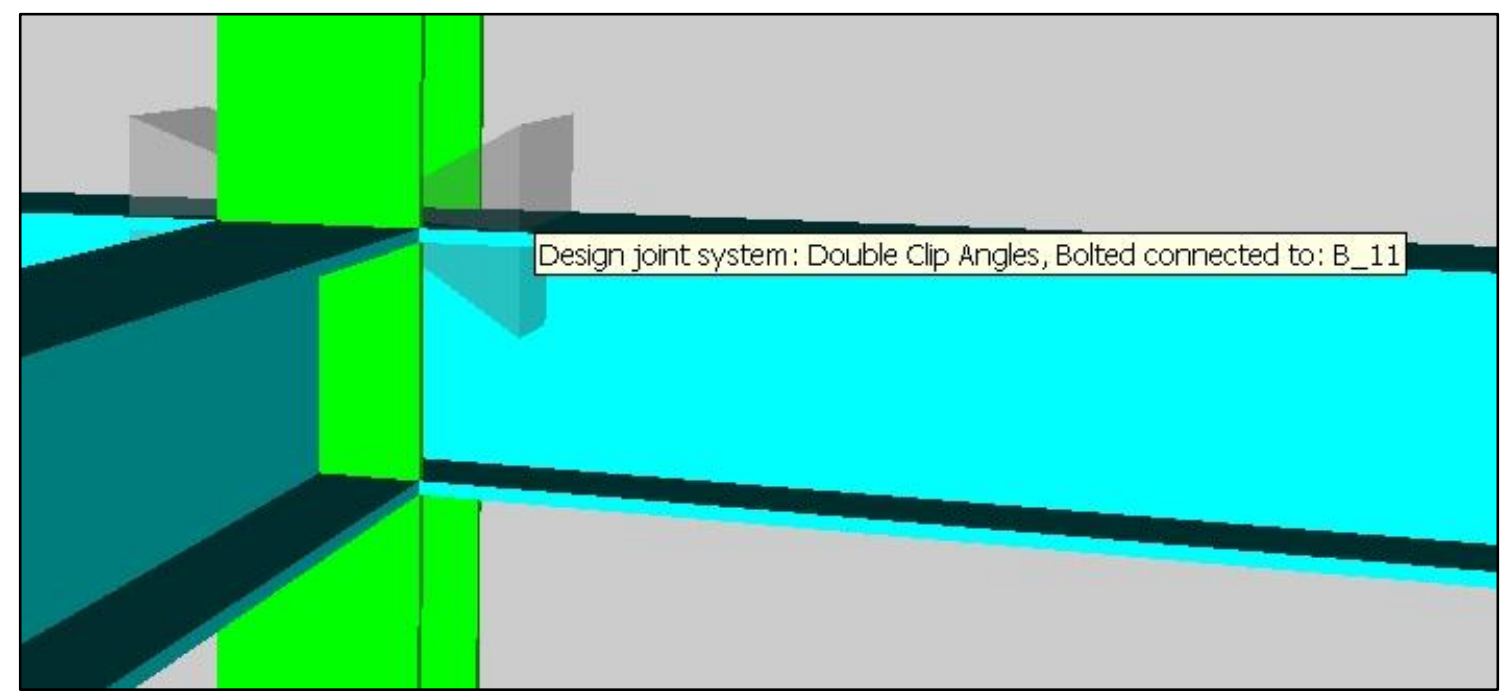

Figure 44: Design joint system 


\section{Other Features}

The options for the features described in this section can be found in the Other, VRML, and More VRML tabs.

\subsection{Grid Lines}

If grid lines are included in the CIS/2 file, they can be displayed in the VRML model by selecting the option for Grid Lines in the Other tab. Figure 45 shows a VRML model with orthogonal and radial grid lines.

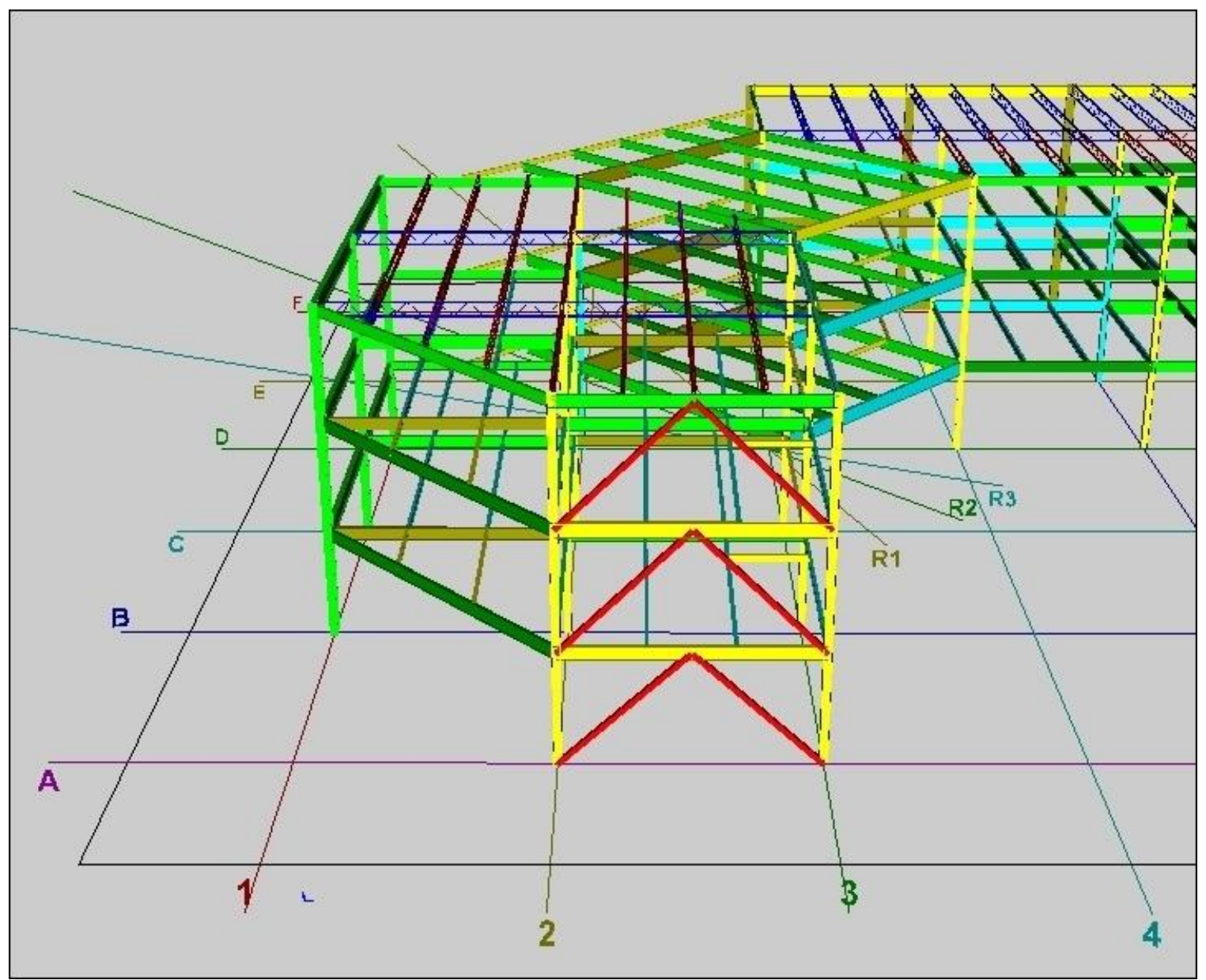

Figure 45: Orthogonal and radial grid lines

CIS/2 files can also contain grid levels as shown in Figure 46. The grid levels appear as lines on the left and back sides of the VRML model and typically represent the elevation of that level. Buttons also appear in the VRML model to toggle on and off the grid lines and grid levels. Some CIS/2 files have so many grid lines and levels that displaying them in the VRML model creates too much clutter in the display. 


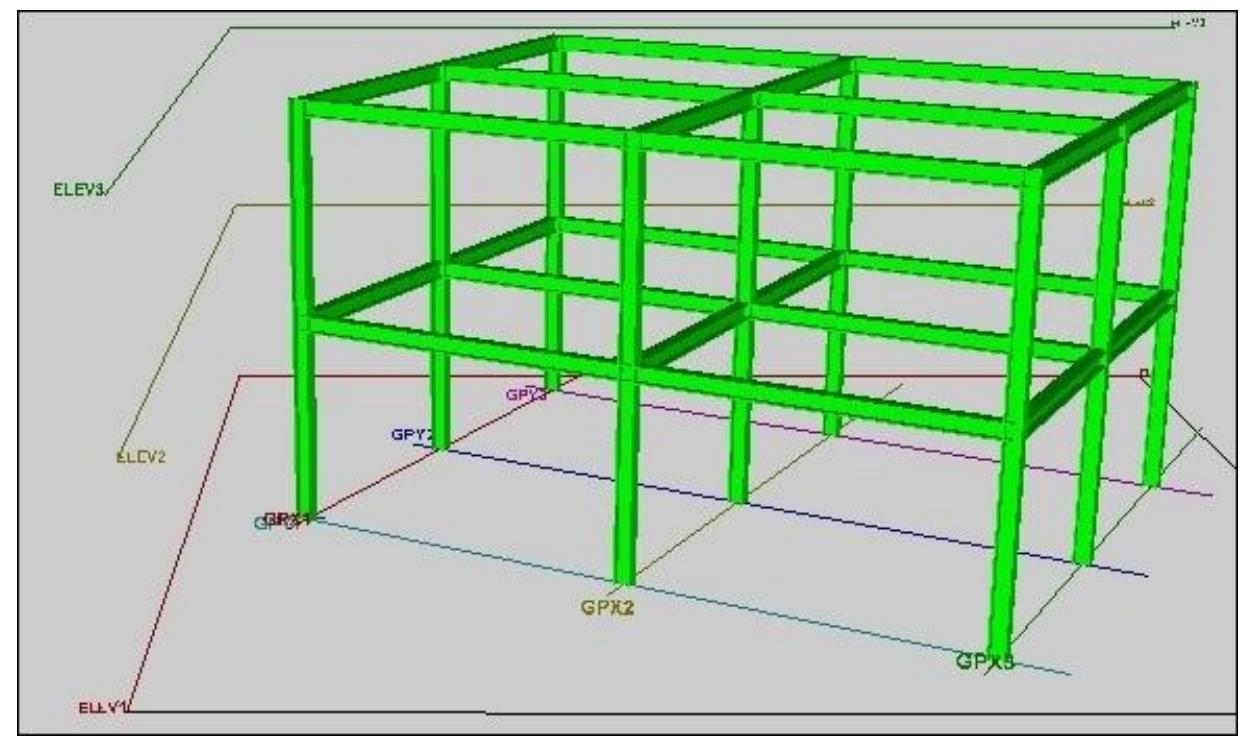

Figure 46: Grid lines and levels

\subsection{Vertical Axis}

For almost all CIS/2 models, the vertical axis of the model is the positive $\mathrm{Z}$ axis. If the vertical axis of the model is the positive Y axis, it can be selected with the Vertical Axis option in the Other tab.

\subsection{Model Types}

A CIS/2 file can contain a design, analysis, or detailed model. Some CIS/2 files contain multiple models, i.e. design and analysis models. If the CIS/ 2 file contains multiple models, then the VRML model will contain buttons to toggle on and off each model as shown in Figure 47. In many cases the design and analysis model will look the same. If only one of the model types is of interest, then the model type that is translated can be selected with the Model Type option in the Other tab. When the CIS/2 file is translated, the model type is reported in the Status tab (section 3.3 above).

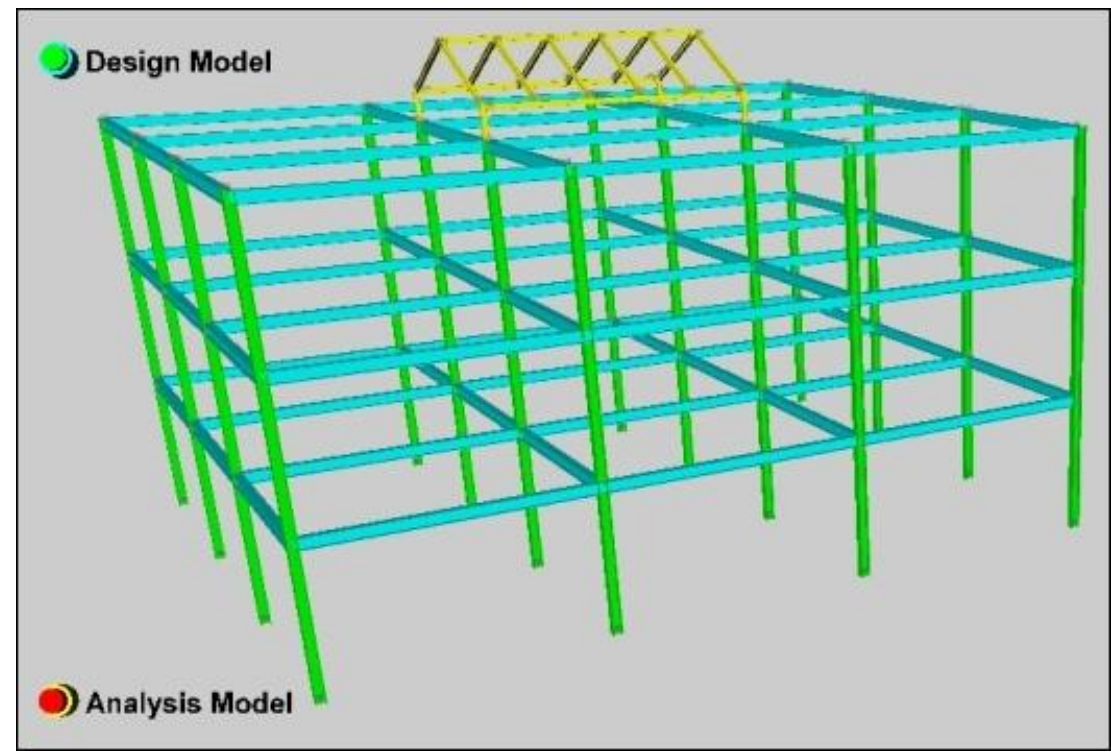

Figure 47: Model with design and analysis models 


\subsection{Curved Parts}

Curved parts also include bent plates and corrugated decking (see Figure 7). The geometry of the curve for a curved part can be represented in many ways in a CIS/2 file. SteelVis only supports curves defined by a polyline or a b-spline. Other types of curve geometry are ignored and the part will appear straight.

Plates and slabs with curved edges defined by curves other than a polyline will display the curved edges as straight.

How the curved part appears in the VRML model also depends on the VRML plugin or viewer. Use the VRML Plugin option in the VRML tab to select which VRML plugin is used to display the VRML model. If the VRML plugin is not listed, select the Other option.

\subsection{Navigation Mode}

The navigation mode refers to how the user rotates, navigates, and interacts with the VRML model in the VRML plugin or viewer. The navigation mode can be selected with the Navigation Mode option in the VRML tab. The Examine mode treats the VRML model as if it were an object that the user can hold in their hands. The actual interaction with the VRML model depends on the VRML plugin or viewer.

\subsection{Merging Models}

Merging VRML models is a way to automatically include another VRML model with the one generated by SteelVis. Figure 48 shows the options for merging VRML models in the VRML tab. To enable this option, select Merge File and Browse for the VRML file to be merged. The Merge Scale, Translation, and Rotation are applied to the selected VRML file to align it with the VRML model generated by SteelVis. The Do Not Center VRML model option is useful to help align the two VRML models. By default, the VRML model generated by SteelVis is always centered about 0,0,0.

The rotation is specified by four values. The first three numbers are an axis about which the model is rotated. The fourth number is the rotation angle in radians. The selectors to the right of the rotation value can be used to select typical axis and rotation angles in degrees which are converted to the required four rotation values.

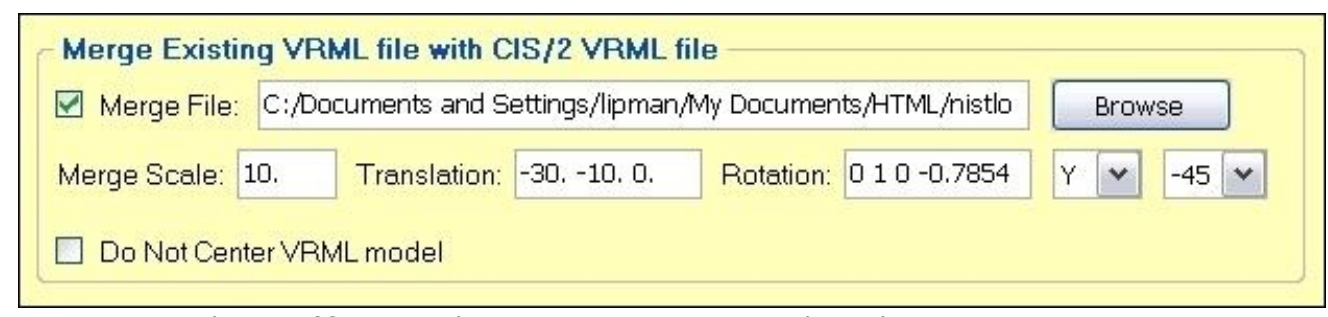

Figure 48: Merging VRML model options in the VRML tab

Figure 49 shows an example of merging a VRML model of the NIST logo with the VRML model generated by SteelVis. Buttons appear in the VRML model to toggle on and off the merged VRML model and the CIS/2 model.

A screenshot of another example of merging VRML models can be found in the Websites menu under VRML Information. In that example, a VRML model of the adjacent buildings, equipment, and terrain was merged with the VRML generated by SteelVis to show the structural steel model in the context of its surroundings. 


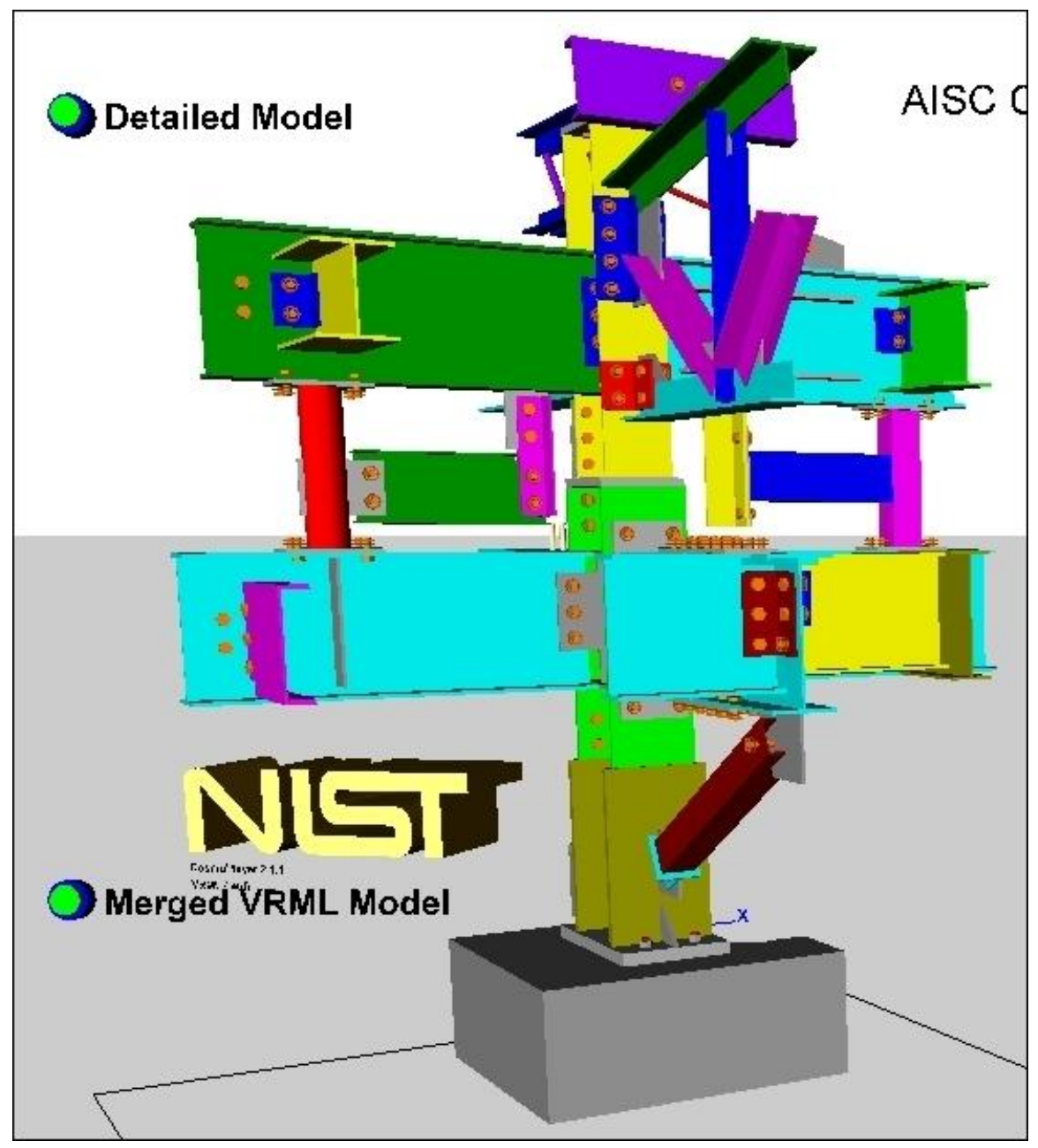

Figure 49: Merged VRML models

\subsection{X3D and X3DOM}

X3D is the successor to VRML and is encoded using the Extensible Markup Language (XML) [8]. Many of the VRML plugins and viewers also can display X3D files. X3DOM is a way to embed an X3D file directly in a web page and display the 3D model in a web browser without a VRML or X3D plugin or viewer [9]. X3DOM files generated by SteelVis can only be displayed in web browsers that support WebGL such as current versions of Firefox, Google Chrome, or Internet Explorer 9 with Flash 11. For Internet Explorer 9 all Compatibility View settings must be disabled. Information about generating X3D and X3DOM files can be found in the SteelVis Help menu. A link to a sample X3DOM file generated by SteelVis can be found in the Websites menu under CIS/2 Information. Some of the features of the VRML model generated by SteelVis are not supported by X3DOM.

The options to generate an X3D or X3DOM file are in the More VRML tab. SteelVis uses the InstantReality Framework to generate X3D and X3DOM files. A link to the InstantReality website can be found in the Websites menu under VRML Information. After InstantReality is downloaded and installed, then X3D and X3DOM files can be generated. InstantReality also includes a VRML and X3D viewer called Instant Player that will show up in the Browsers menu (section 3.1.2 above). 


\subsection{Simple VRML}

The VRML that is generated by SteelVis is highly optimized to minimize the VRML file size and the computations required to generate the geometry in a VRML plugin or viewer. However, some software that displays VRML files does not support the optimized VRML generated by SteelVis. In this case, use the Simple VRML option in the More VRML tab which will generate simple non-optimized VRML that can be imported by almost any software.

The other options related to the VRML node name and embedding VRML in a 3D PDF file are described in the Help menu and tooltip help. Figure 50 is an example of a VRML model embedded in a PDF document. Click on the model to rotate it and to access other features of the 3D model. The interactive features of the model are only available in Adobe Reader. Other examples of VRML models embedded in 3D PDF files can be found in the Websites menu under CIS/2 Information.

\subsection{VRML PROTOs}

VRML PROTOs are programming scripts written in JavaScript that are included in the VRML model to provide added functionality. PROTO is short for Prototype. For example, in many if the examples above, the buttons that toggle on and off parts of the VRML model are generated with a VRML PROTO. The use of VRML PROTOs by SteelVis in VRML models is transparent to the user.

However, there is one display option that requires using the VRML with PROTOs option in the More VRML tab. That option is the rendering option for "Shaded, transparent, edge, and wireframe" in the Rendering tab (section 4.2 above and Figure 16). The use of VRML PROTOs also creates the buttons to toggle on and off the bolts, holes, and welds.

The VRML with PROTOs option is not recommended for very large CIS/2 models. For some VRML plugins, viewers, and other software that can display VRML files, parts of the VRML model that are displayed with PROTOs will not be visible. 


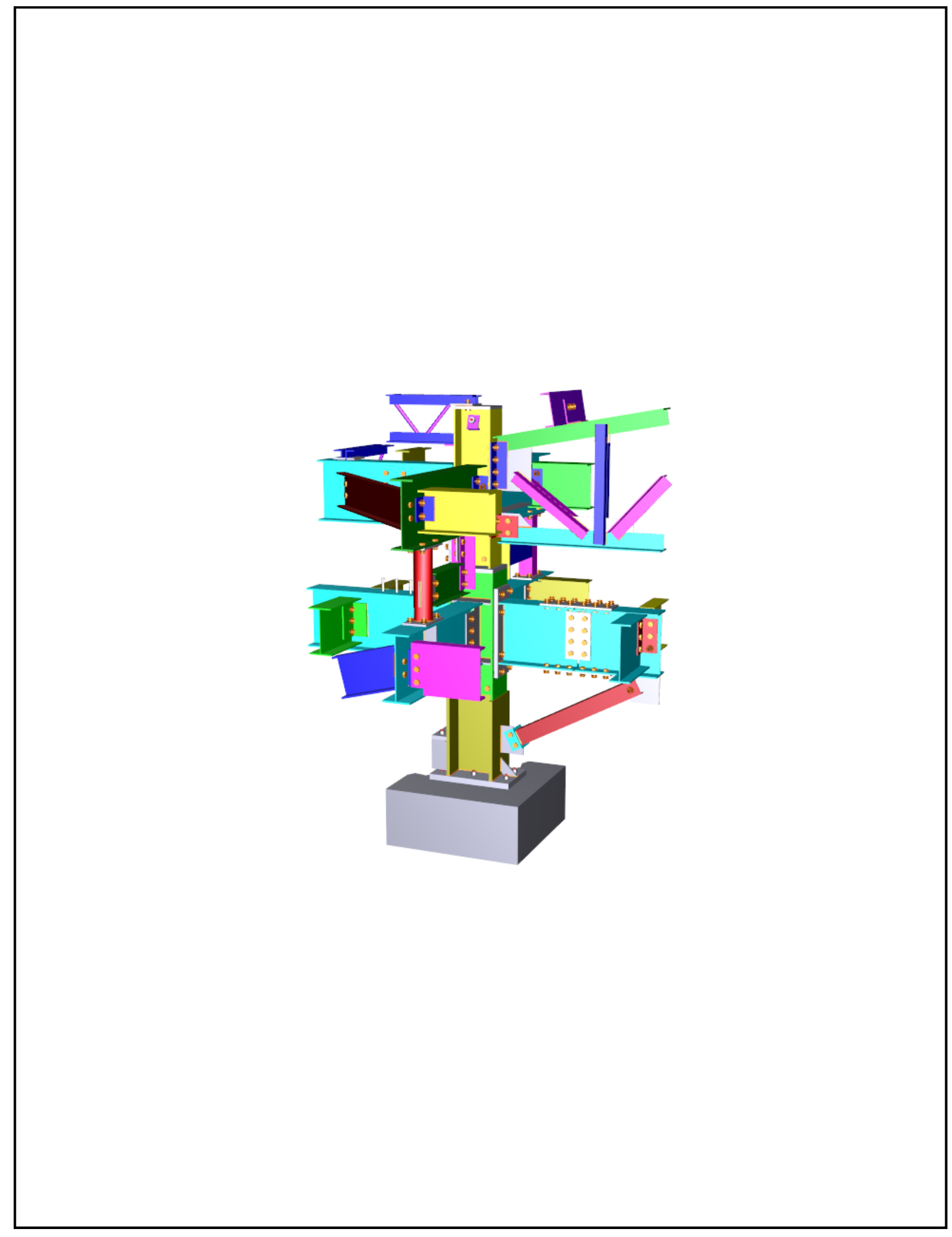

Figure 50: Interactive 3D model 


\section{Debugging Features}

Most of the debugging features can be found in the Debug menu as shown in Figure 51. Two other debugging options can be found in the Reports and Detailed tabs.

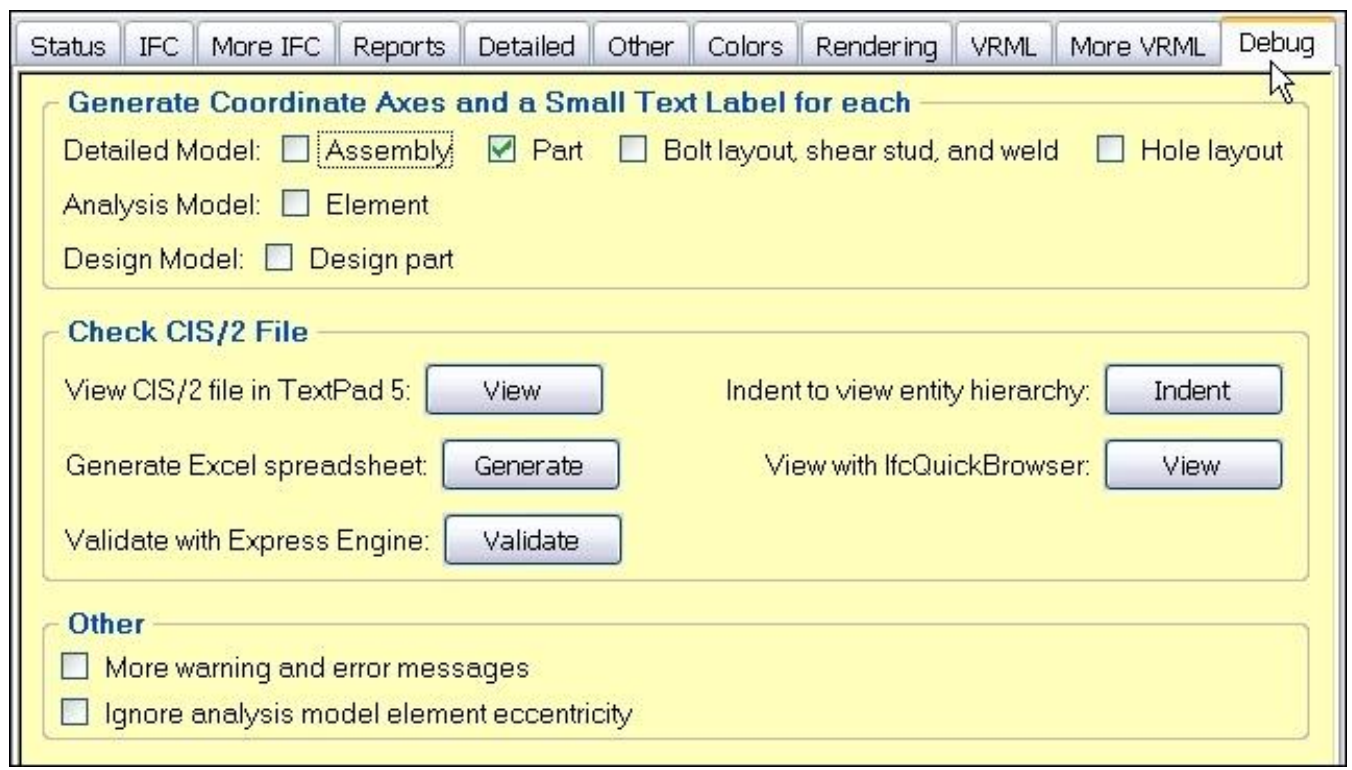

Figure 51: Debug tab options

\subsection{Coordinate Axes}

Coordinate axes can be included in the VRML model for any assembly, parts, bolt layout, weld, hole layout, analysis element, or design part. They are useful to confirm the absolute and relative position and orientation of items in the VRML model. The coordinate axes are displayed at the position where the item is located in the CIS/2 model and includes a text label. The text label rotates so that it always faces forward and contain information relevant to that item.

Figure 51 shows coordinate axes for an assembly and a transparent beam and plate. The assembly coordinate axes are black and the two part coordinate axes are blue. Figure 52 shows the coordinate axes for two angle brackets and a bolt layout. The part coordinate axes are blue and the bolt layout coordinate axes are red.

\subsection{CIS/2 File Checking}

Several options are provided to check the contents of the CIS/2 file. Some of the options depend on other optional software. At a minimum in the Check CIS/2 File of the Debug tab, the two options in the first row of that section will be available. The "View" button will display the CIS $/ 2$ file in a text editor and the "Indent" option will also display the CIS/2 file in a text editor, however, the CIS/ 2 entities will be rearranged and indented to show the hierarchy of information in the CIS/2 file.

Figure 54 shows a sample of the output using the "Indent" option. In this example, it is easy to see how the definition of the axis (\#20=AXIS2_PLACEMENT_3D) refers to the cartesian point (\#35) and two directions (\#33, \#32) and how the axis is referred to by the design part (\#14) through the coordinate system (\#17). 

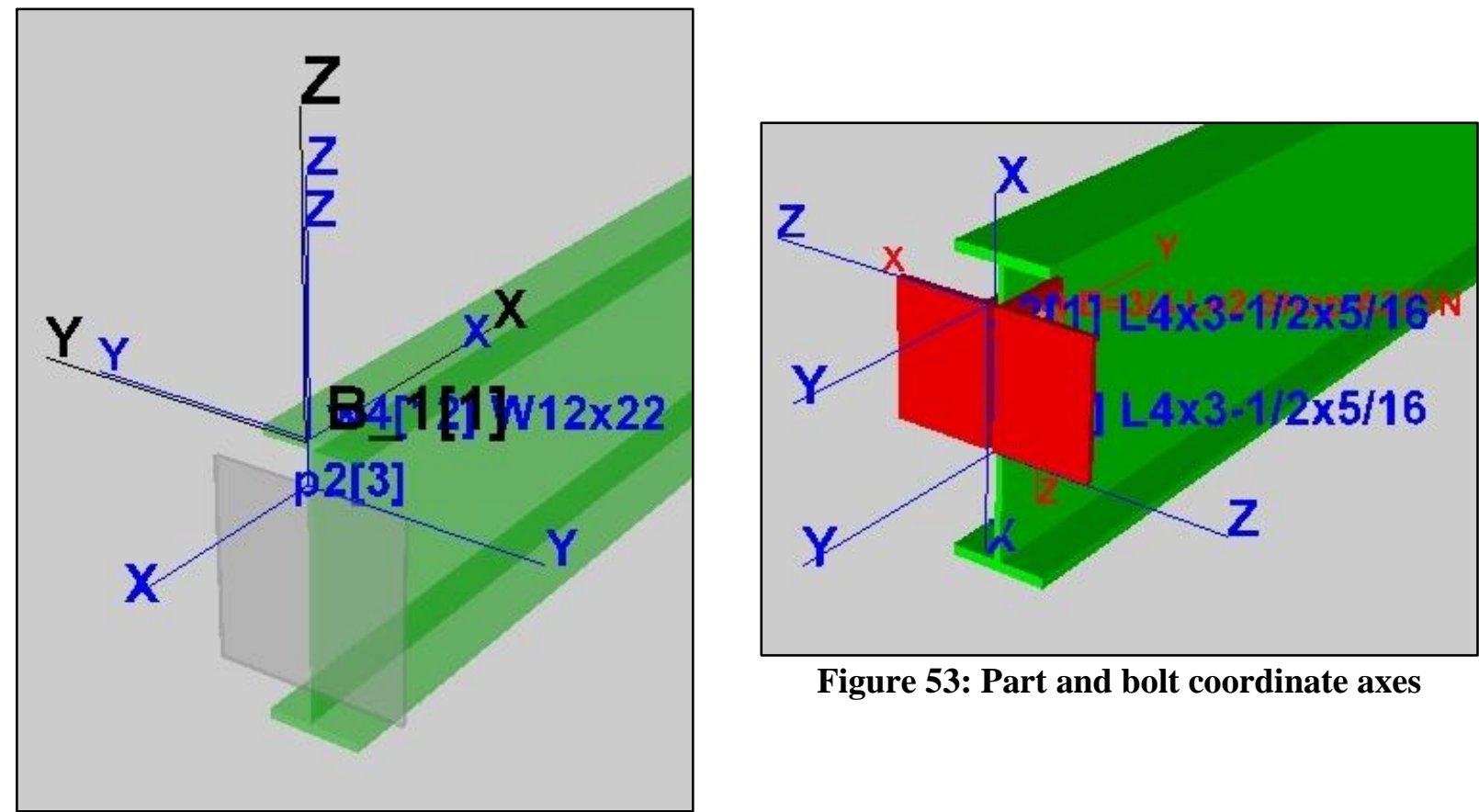

Figure 53: Part and bolt coordinate axes

Figure 52: Assembly and part coordinate axes

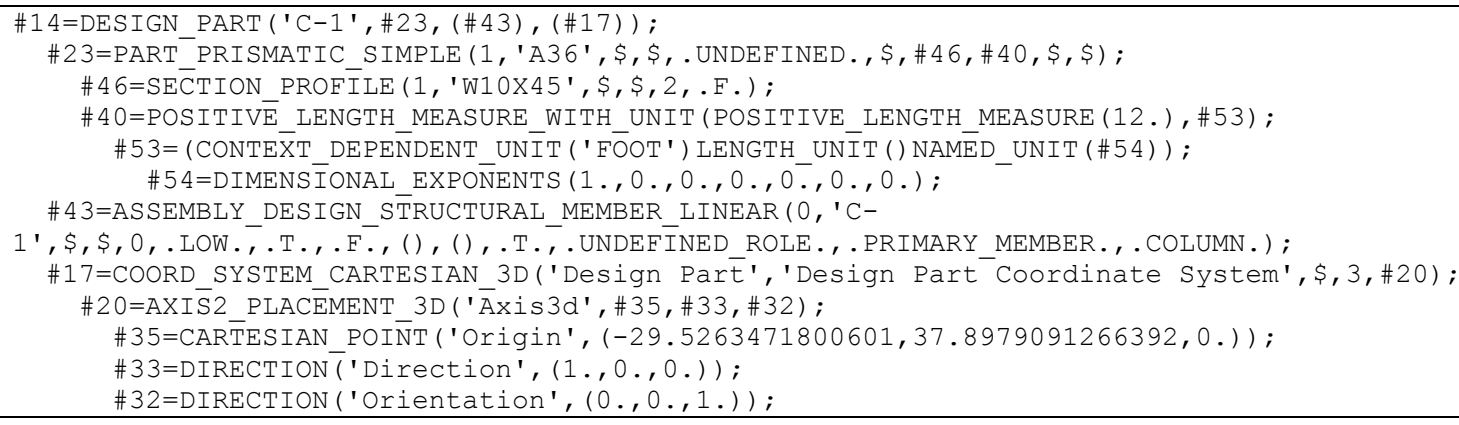

Figure 54: CIS/2 file with indentation

The options in the second row of the Check CIS/2 File section are to "Generate" an Excel spreadsheet from the CIS/2 file and to "View" the CIS/2 file with the IfcQuickBrowser. The IfcQuickBrowser is a free IFC file browser that also works with CIS/2 files [10].

\subsection{Generate Excel Spreadsheet}

To generate an Excel spreadsheet from a CIS/2 file, the CIS/2 File Analyzer (CIS2_Excel . exe) must first be setup. The CIS/2 File Analyzer uses IFCsvr [11] which is a program that can read and process IFC files that also works with CIS/2 files. The Analyzer will check for IFCsvr and install it, if it is not already installed. Any version of Microsoft Excel is required to generate a spreadsheet. Running the Analyzer the first time will create an icon on the desktop for "CIS2 File Analyzer". The CIS/2 File Analyzer is based on the IFC File Analyzer [12, 13].

Figure 55 shows the user interface for the CIS/2 File Analyzer. Options are available to selectively process different types of CIS/2 entities as shown in Figure 56. Sometimes the Analyzer will not be able to completely process a CIS/ 2 file due to a problem in the file. In that case, the feedback in the Status tab 
will show at which entity type the processing stopped. In the Options tab, deselect that type of entity and try generating the spreadsheet again. Typically, the "representation" entity is the cause of the problem.

The spreadsheet generated by the Analyzer creates a summary worksheet as shown in Figure 57. All of the entity types found in the CIS/2 file are listed in Column A starting after row 7. The name of each entity is linked to the individual worksheet for that entity. Column B is the number of each entity type found in the CIS/2 file. Column $\mathrm{C}$ is a link to CIS/2 documentation for that entity.

A worksheet is created for each entity in Column A of the summary worksheet. Figure 58 shows the worksheet for the CIS/2 "material" entity. Cell A1 is the name of the entity and number of that entity. It is also a link back to the summary worksheet. Cell A2 is a link to CIS/2 documentation for that entity. The CIS/ 2 entity ID is in Column A starting with row 4. Row 3 starting with column B has the names of all of the attributes for the "material" entity. Each row starting with row 4 contains the values of the attributes for each of the nine "material" entities. Similar worksheets are created for every CIS/2 entity type.

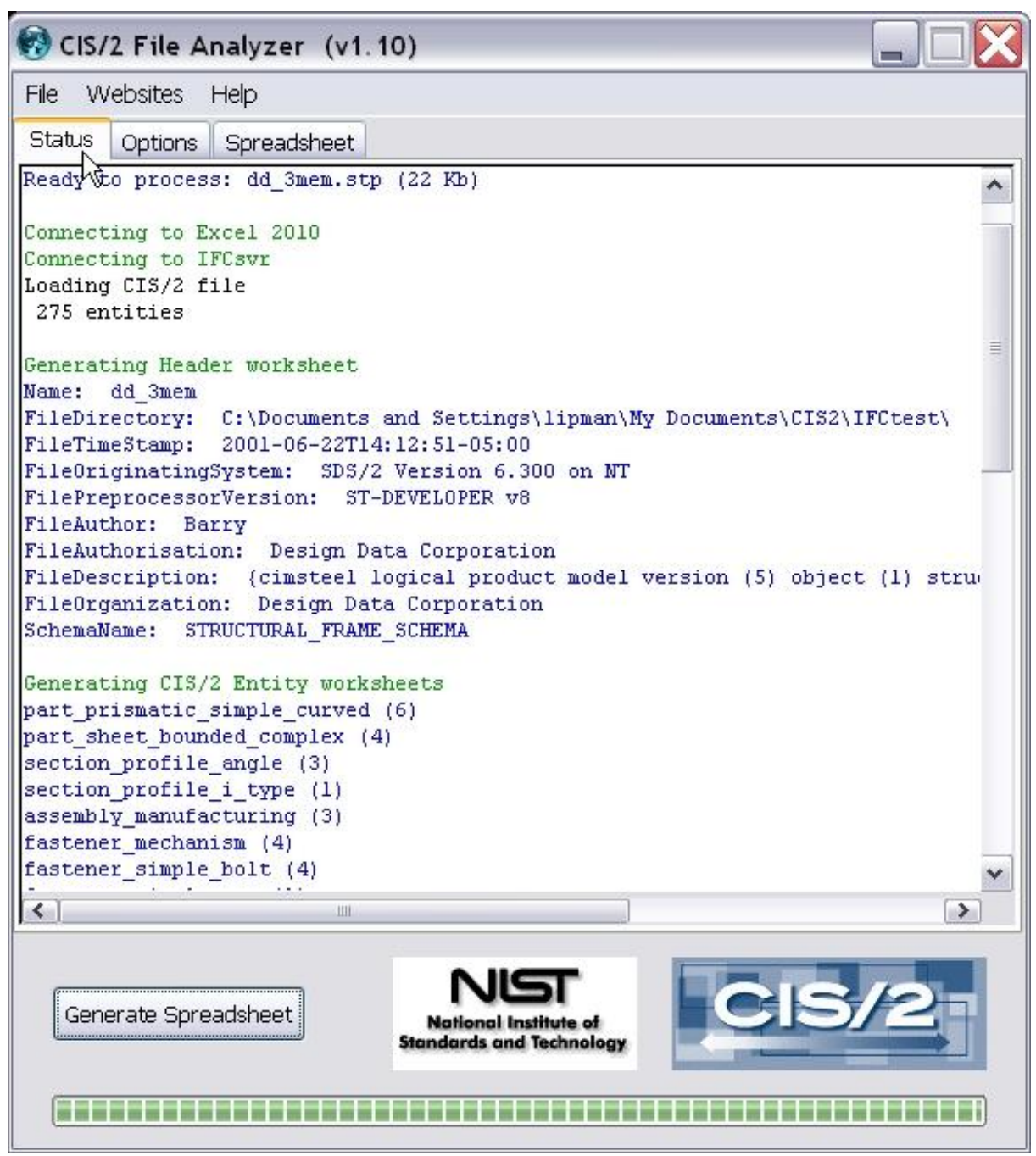

Figure 55: CIS/2 File Analyzer user interface 


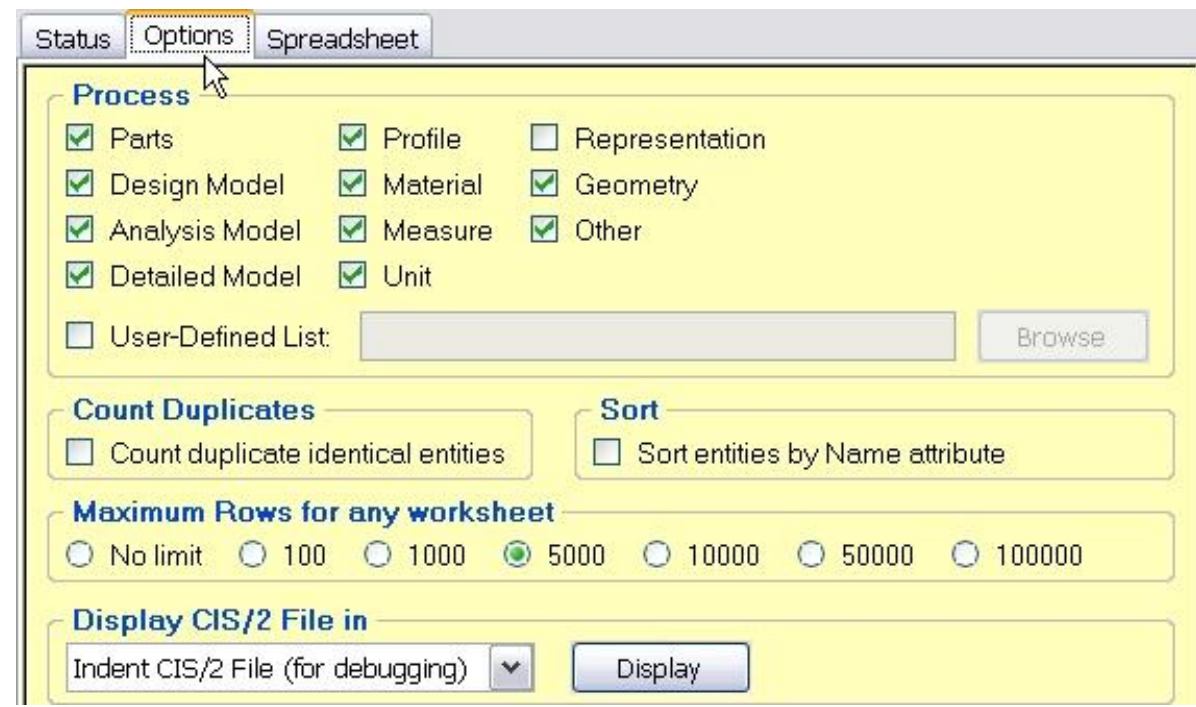

Figure 56: CIS/2 File Analyzer options tab

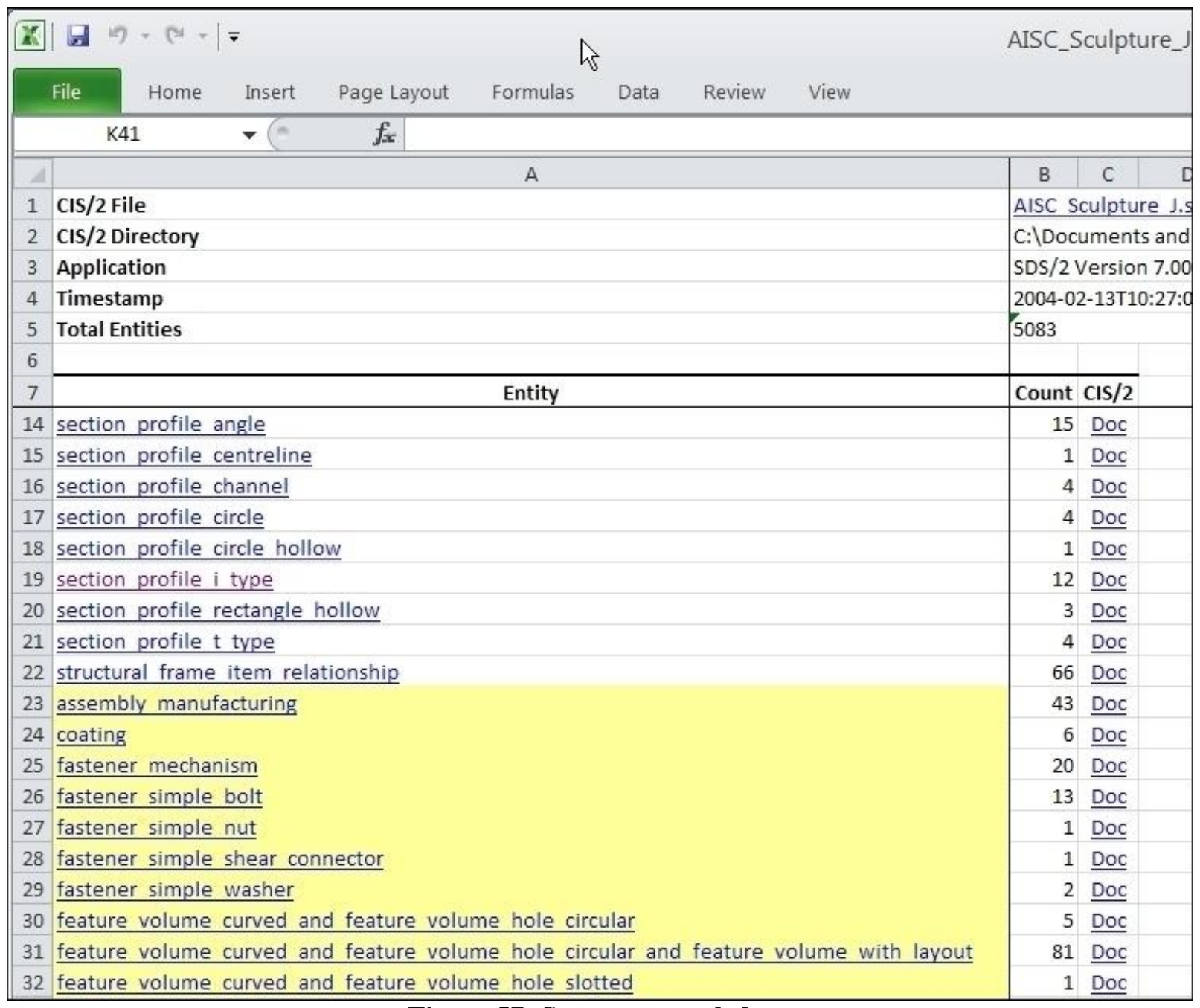

Figure 57: Summary worksheet 


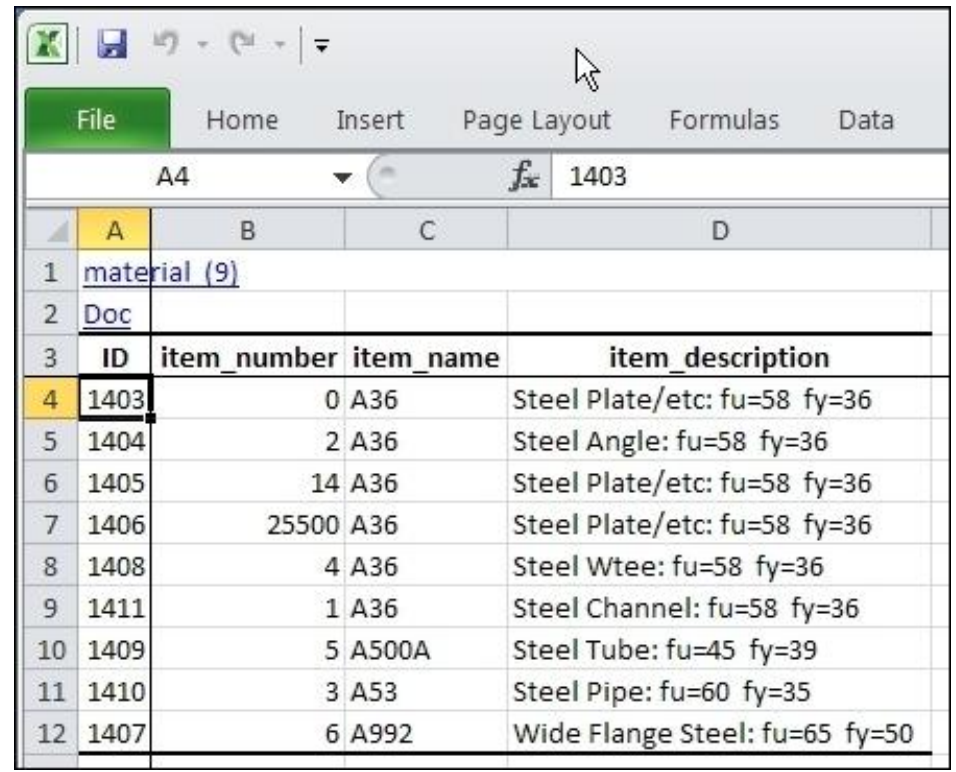

Figure 58: Worksheet for the material entity

\subsection{File Validation}

The option in the third row of the Check CIS/2 File section is to "Validate" the CIS/2 file with Express Engine. Although many syntax errors are highlighted in red in the Status tab (section 3.3.1 above), SteelVis does not validate that the CIS/2 file conforms to the CIS/2 schema. Use the option for 'More warning and error messages' in the Debug to report specific CIS/2 entities that are the cause of some syntax errors.

Checking for conformance to the schema can be done with several commercial tools, such as EDM Supervisor and STEP Tools, and with free tools such as Express Engine [14] that was formerly known as NIST Expresso [15]. When Express Engine is installed, the option to "Validate" the CIS/2 file with Express Engine will appear in the Debug tab. When using this option, if the CIS/2 file is named mycis2file.stp, then the Express Engine output will be written to the file mycis2file_ee. log. More information about using Express Engine can be found in the Help menu under Validating CIS/2 Files. 


\section{Generating an IFC File}

A CIS/2 file can be translated to an IFC file so that the structural steel model can be imported to BIM applications that support IFC files. The capability to translate CIS/2 files into IFC files is based on research at NIST to develop a mapping between the information, entities, and attributes of each file type $[16,17]$. SteelVis implements the mapping described in the research, however, the most important characteristic of translating a CIS/2 file to an IFC file is that there is no single correct way to do it. Any combination of IFC options described below will result in a valid IFC file, however, the BIM application importing the IFC file might not support how the steel structure is modeled in the IFC file. Support depends on how information in the resulting IFC file is mapped to the internal representation of those features in the BIM application.

When translating from a CIS/2 file to an IFC file, there is also a loss of some of the semantic meaning from the CIS/2 file. This is most common for CIS/2 detailed models. For example, in CIS/2, a group of bolts is parametrically defined by their pattern or layout in an assembly and copes are parametrically defined by their dimensions and position on a part. However, in IFC there is no corresponding parametric definition of bolt patterns or copes. SteelVis generates the correct IFC geometry for bolts and copes, however, their parametric definition is lost in the translation. Many of these differences are described in the NIST research for mapping CIS/2 to IFC described above.

The two most important options for generating an IFC file are: (1) how geometry is represented in the IFC file (see Shape Representation in section 9.1 below) and (2) how elements are represented in the IFC file as beams, columns, and other structural members (see Element Representation in section 9.2 below).

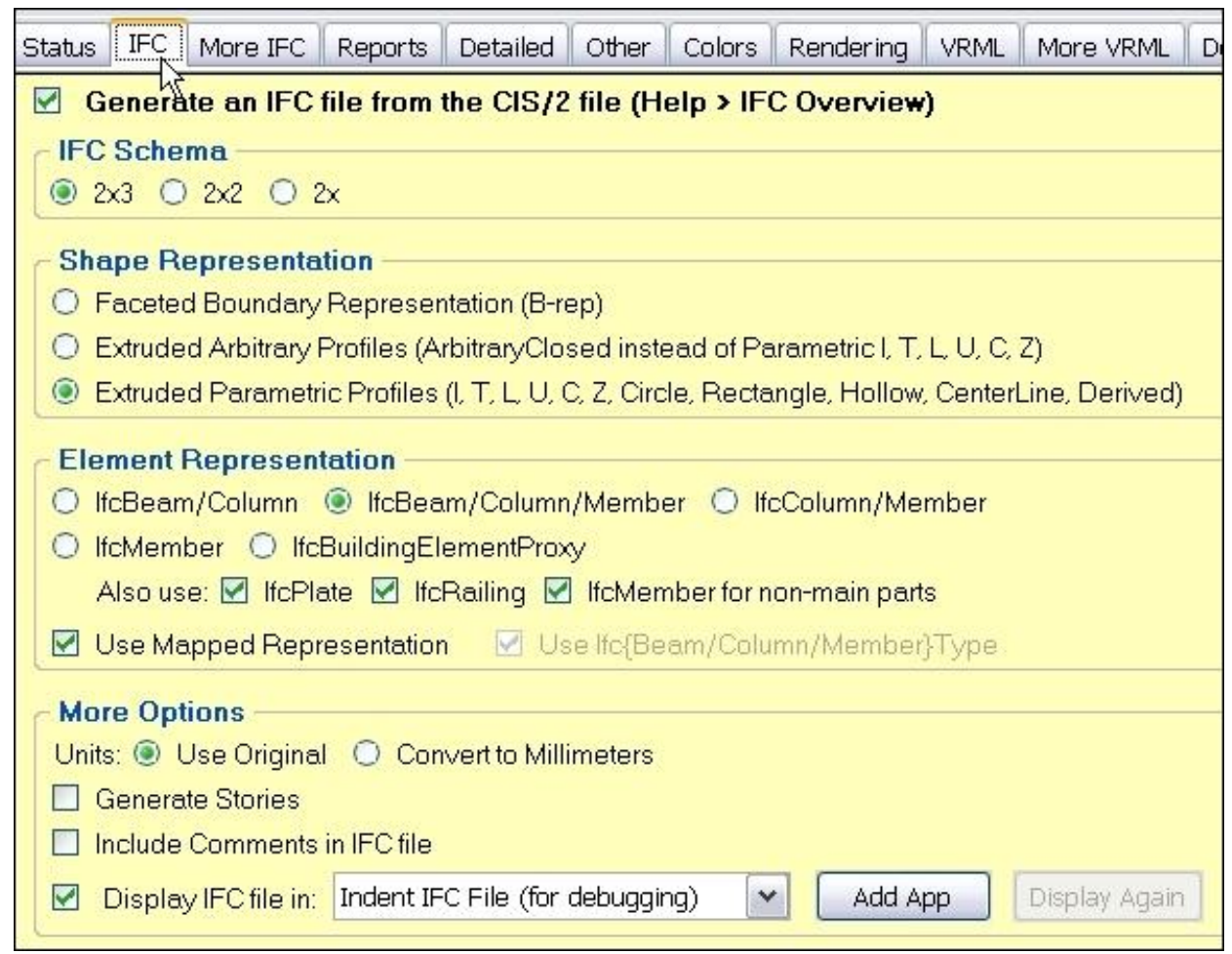

Figure 59: IFC file generation options

The main options to generate an IFC file are found in Figure 59. All of the IFC options are enabled when "Generate an IFC file from the CIS/2 file" is selected. The first option is to select the version of the IFC 
schema. Version $2 \times 3$ is the current version of the IFC schema and should be selected as it is supported by almost all IFC applications and BIM software.

An IFC file is generated when either of the Translate buttons is used (section 3.4 above). If the IFC options are changed, the IFC file cannot be updated without translating the CIS/2 file. Warning and error messages about generating the IFC file are highlighted in light blue in the Status tab (section 3.3.1 above).

\subsection{Shape Representation}

Shape representation refers to how geometric shapes are represented in an IFC file. The distinction is between what the part is versus how the shape of the part is modeled. For example, in CIS/2 a part is defined by a cross section (i.e. W14X120) and length (i.e. 3.048 meters), however, the geometric representation of the part is not specified.

IFC takes a different approach. All parts must explicitly define how the geometry of the part is generated. Two methods are used in IFC to model geometry: (1) by modeling all of the surfaces of a part or (2) by extruding a section along a path. The following describes the methods for Shape Representation in the IFC options tab.

- Faceted Boundary Representation (B-rep) - geometry is modeled by all of surfaces (facets) on the outer boundary of a part

- Surfaces are defined by their edges which are defined as a line between two points.

- For example, a straight part with a rectangular cross section will be modeled as six surfaces, twelve edges, and eight points.

- All straight parts can be modeled with this method.

- All copes (cutouts) can be modeled with this method.

- Curved parts, bent plates, and corrugated deck are not modeled by SteelVis with this method, see section 9.1.1 below.

- Extruded Arbitrary Profiles - geometry is modeled by extruding an arbitrarily shaped section profile along a path

$\circ$ An arbitrary profile is defined by the points and edges that define the outline of a cross section.

- For example, the profile for a rectangular cross section is defined by four points and the edges between them.

- Ignoring the leg slope and fillet radius, the profile for an angle is defined by six points and the edges between them.

- The path along which the profile is extruded can be straight or curved.

$\circ$ Only miter cuts can be modeled with this method, all other copes (cutouts) are not modeled.

- Supported by the IFC Coordination View [18].

- Extruded Parametric Profiles - geometry is modeled by extruding a parametrically defined section profile along a path

- A parametric profile defines the dimensions of a cross section by its depth, width, and thickness.

- For example, an I-shaped cross section is defined by its depth, width, flange thickness, and web thickness.

- Parametric profiles include: I-shape, T-shape, angle, channel, zee, circular, rectangular, hollow, and centerline sections. 
- Centerline section profiles can be used to model bent plates, corrugated decking, and cold-formed steel.

- The path along which the profile is extruded can be straight or curved.

$\circ$ Only miter cuts can be modeled with this method, all other copes (cutouts) are not modeled.

- Supported by the IFC Coordination View [18].

\subsubsection{Shape Representation Limitations}

The most significant limitation regarding shape representations is that SteelVis cannot represent curved parts, bent plates, and corrugated decking as a faceted boundary representation. Those types of parts will always be modeled as an extruded parametric profile. For any type of shape representation only the top and bottom chords of a joist are written to the IFC file.

Another limitation is that curved parts, bent plates, corrugated decking, mirrored sections, double angles, and joists, modeled as extruded parametric profiles might not be supported by many BIM applications that import IFC files. Applications that do not correctly import those types of parts will display them incorrectly or not at all.

To test if a BIM application can successfully import any type of structural member modeled with any of the methods described above, use the three cis2ifc_test*.stp sample CIS/2 files (section 2.2 above). Compare the appearance of the resulting VRML model with the appearance of the IFC file in the BIM application or IFC viewer to see if a particular shape representation is supported.

\subsection{Element Representation}

In a CIS/2 file, the function of a part (i.e. beam, column, brace, etc.) can be defined by an optional text string. There is no requirement that the text string be used or what type of information is in the text string.

Again, IFC takes a different approach. Any part in an IFC file must be explicitly defined as a beam, column, or other type of structural member. The options for element representation provide a variety of methods to assign parts in a CIS/ 2 file to IfcBeam, IfcColumn, or IfcMember IFC entities. The IFC documentation [19] provides the following definitions for those entities:

- IfcBeam - "An IfcBeam is a horizontal, or nearly horizontal, structural member. It represents such a member from an architectural point of view. It is not required to be load bearing."

- IfcColumn - "An IfcColumn is a vertical structural member which often is aligned with a structural grid intersection. It represents a vertical or nearly vertical, structural member from an architectural point of view. It is not required to be load bearing."

- IfcMember - "An IfcMember is a structural member designed to carry loads between or beyond points of support. It is not required to be load bearing. The location of the member (being horizontal, vertical or sloped) is not relevant to its definition (contrary to IfcBeam and IfcColumn)." 
The IFC element representation options are shown in Figure 59 above. The assignment of CIS/2 structural members to the different IFC element representations is based on the orientation (horizontal, vertical, other) of that member or assembly:

- IfcBeam/Column - IfcColumn is used for all vertical structural members, everything else is an IfcBeam.

$\circ$ For detailed models, the assignment of IfcBeam or IfcColumn is based on the orientation of the assembly and not the part. Therefore for a horizontal beam assembly, all parts would be IfcBeam, including angle brackets at the end of the beam whose longitudinal axis is vertical and could be considered an IfcColumn based on their orientation.

- IfcBeam/Column/Member - IfcColumn is used for all vertical structural members, IfcBeam is used for all horizontal structural members, and IfcMember is used for all other structural members such as diagonal bracing.

- For detailed models, the assignment of IfcBeam, IfcColumn, or IfcMember is based on the orientation of the assembly and not the part as described above.

- IfcColumn/Member - IfcColumn is used for all approximately vertical structural members and 9.1

- IfcMember - Everything is an IfcMember.

- IfcBuildingElementProxy - Everything is an IfcBuildingElementProxy element.

- This can be used with IFC applications that do not support IfcMember.

- IfcPlate - If plates can be identified in the CIS/2 file, then IfcPlate is used for those members.

- IfcPlate might not be supported by all IFC applications.

- IfcRailing - If railings can be identified in the CIS/2 file, then IfcRailing is used for those members.

- IfcRailing might not be supported by all IFC applications.

- IfcMember for non-main parts - If non-main members can be identified in the CIS/2 file, then IfcMember is used for those parts (section 6.1.4 above).

The option for "Use Mapped Representation" is a method in an IFC file to reuse common parts. This reduces the size of the IFC file and the amount of processing by an IFC application. 


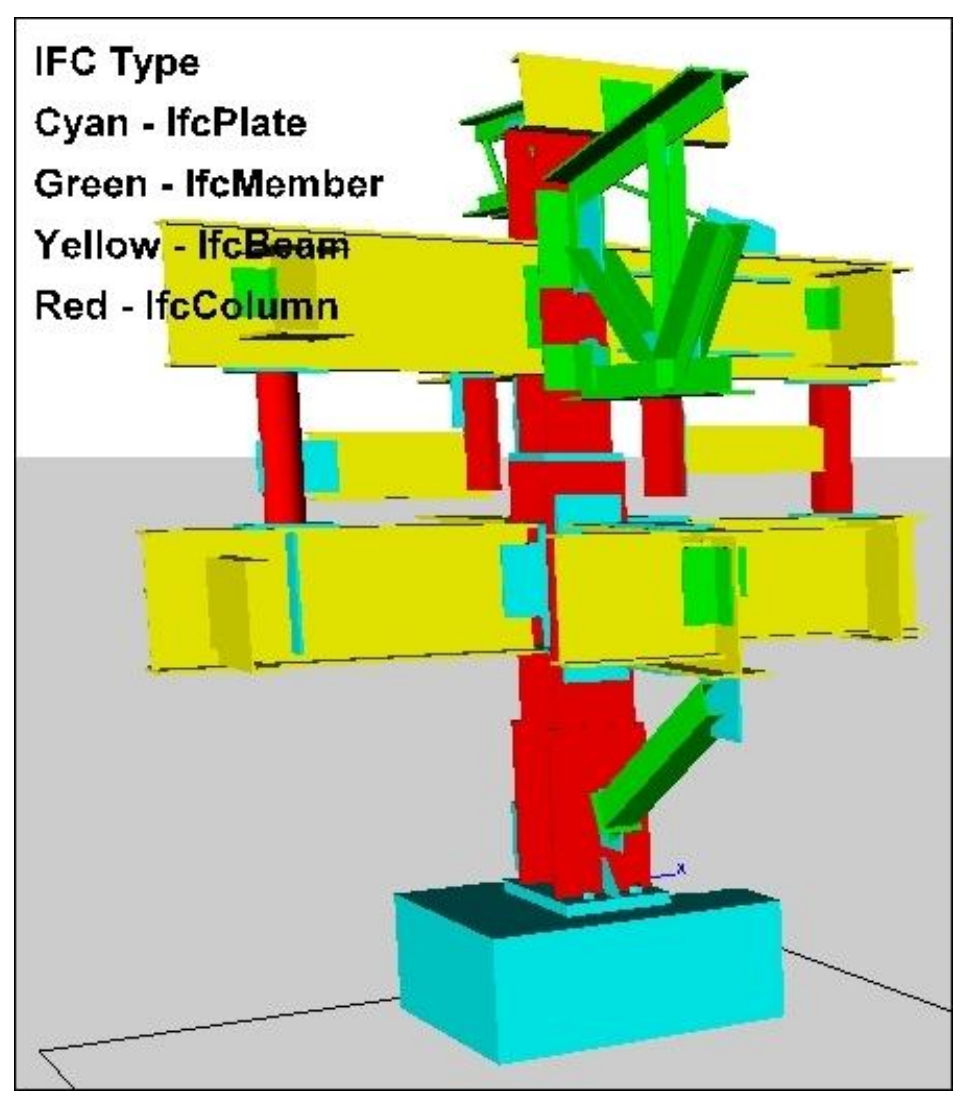

Figure 60: Structure colored by IFC type (element representation)

Figure 60 shows a structure colored using the Color by IFC options (section 4.1 above) using the options for element representation shown in Figure 59. The Color by IFC option is useful to see the effect of using any of the IFC element representation options.

\subsection{Units}

The length units used in the IFC file can be either the same units as the CIS/2 file or all of the length units can be converted to millimeters. Converting all units to millimeters is useful when the CIS/ 2 file contains multiple length units such as feet and inches.

\subsection{Stories}

IFC has the concept that structural members can be associated with a building story (floor), however, CIS/2 does not. Therefore all structural members will be contained in one story if stories are generated. The only exception is CIS/ 2 files generated by RAM Steel that use a text string to identify which story a member is located on.

\subsection{Displaying the IFC File}

After an IFC file is generated from a CIS/2 file, the IFC file can be displayed automatically in many BIM applications and IFC viewers by selecting the "Display IFC file" option as shown in Figure 59 above. The list of options that appears in the drop-down menu contains BIM applications and IFC viewers that are either installed in their default location, i.e. C:IProgram Files, or have a shortcut to them on the desktop. At a minimum, the drop-down menu list will contain the WordPad text editor and a program to "Indent IFC File". This program is similar to the option for indenting CIS/2 files (section 8.2 and Figure 54 above). 
Many of the viewers and applications that can appear in the drop-down menu are listed in the Websites menu under IFC Viewers and Applications. For some viewers and applications in the drop-down menu, the user has to manually import the IFC file.

The "Add App" button can be used to add other applications to the drop-down menu list. The "Display Again" button is similar to the "Display Again" button for VRML files (section 3.4 above). The IFC file can be displayed in different IFC applications or viewers in the drop-down menu without having to regenerate the IFC file. Information about displaying the IFC file in Google SketchUp can be found in the Help menu.

\subsection{More IFC Options}

Figure 61 shows more options related to generating an IFC file in the More IFC tab. Some of the options are useful to explore different ways to map CIS/2 information to an IFC file.

\subsubsection{Analysis Model}

If the CIS/2 file contains an analysis model (section 6.2 above), then an IFC structural analysis model can be generated. The structural analysis model can also contain loads and reactions. Some types of CIS/2 analysis model loads are not written to the IFC file. The IFC structural analysis model is only supported by a few IFC applications and viewers. The physical representation of the analysis elements can also be generated from the CIS/2 analysis model and is usually more useful to import to BIM applications.

\subsubsection{Assemblies}

By default, CIS/2 assemblies are written to the IFC file as an IfcElementAssembly which might not be supported in some IFC applications. The other options for IfcGroup and None are enabled only when the IFC file is generated for an older version of the IFC schema.

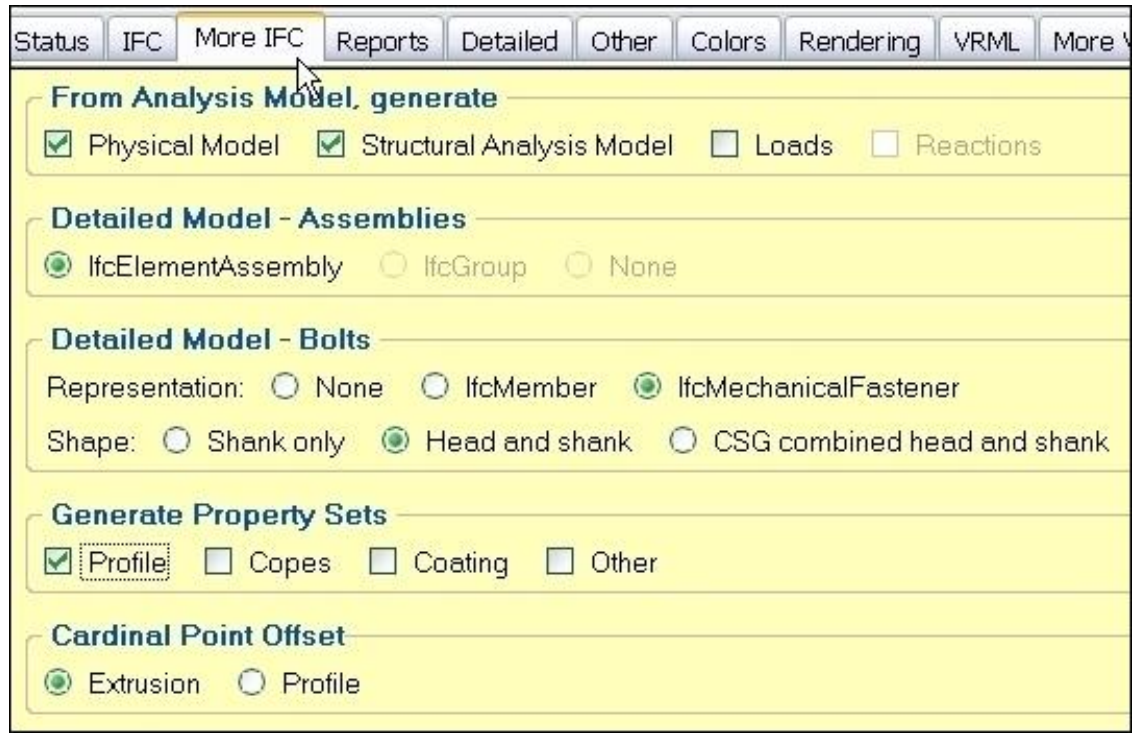

Figure 61: More IFC file generation options 


\subsubsection{Bolts, Welds, and Holes}

Bolts can be written to the IFC file only if bolts are translated in the CIS/2 file (section 6.1.2 above) and a bolt representation of either IfcMember or IfcMechanicalFastener is selected. IfcMechanicalFastener might not be supported in some IFC applications. The shape of the bolt can be represented as either: (1) only the shank of the bolt, (2) the head and shank of the bolt as separate shapes, or (3) the head and shank of the bolt combined into one shape using constructive solid geometry (CSG). The bolt pattern or layout is not written to the IFC file although groups of bolts are related together through IfcBuildingElementProxy.

Since the number of bolts is frequently much larger than the number of parts in a structure, it is not recommended to include the bolts in the IFC file. Although many IFC applications will display bolts, they might not be mapped to the application's internal representation of bolts.

Welds can be written to the IFC file only if bolts and welds are translated in the CIS/2 file (section 6.1.2 above) and bolts are written to the IFC file. It is not possible to write only welds to the IFC file without bolts. IfcFastener is used to represent welds. The weld path is drawn as a line. Most IFC applications do not support importing welds.

Holes and openings are not written to the IFC file.

\subsubsection{Property Sets}

Property sets are a mechanism to extend the information in an IFC file with applications specific values. Options are available to generate property sets related to section profiles, copes, and coatings. The profile property set contains the section name and cardinal point. The copes (cutouts) property set contains parametric values that define copes (section 6.1.1 above). The coating property set contains information about surface treatments. The name attribute used on IfcPropertySet is SteelVis ${ }^{*}$, where $*$ is the name of the type of property. Property sets defined in the IFC schema such as Pset_BeamCommon are not generated.

\subsubsection{Cardinal Point}

The cardinal point defines where the longitudinal axis of a part is located relative to the cross section profile. Locating a section profile for the top-of-steel is done with a cardinal point. There is no cardinal point in IFC $2 \times 3$ although the correct geometry accounting for the cardinal point is written to the IFC file. The geometric offset of the cardinal point can either be associated with the extrusion of the part or the offset of the profile. All IFC applications correctly display the geometric offset modeled with the extrusion option and most with the profile option. The visual appearance of the IFC file should appear the same for either option. The profile property set contains information about the cardinal point.

\subsection{Globally Unique ID}

All building elements in an IFC file have a Globally Unique ID (GUID) assigned to them. The GUID provides a way to uniquely identify a building element and provides the potential to track a building element across multiple software applications. A GUID is a 32 character string, however, IFC files use a compressed version of the GUID that is 22 characters long. The use of a GUID in a CIS/2 file is optional. If the GUID is used, then the entity managed_data_item will appear in the list of translated entities. If possible, the CIS/2 GUID is used as the GUID on the appropriate IFC entity. The 32 character CIS/2 GUID is converted to the 22 character IFC GUID. 


\section{Command-line Version}

Two versions of the command-line (console) version of SteelVis are available (section 2.1 above). ctv . exe runs on any Windows computer and ctv-64 exe only runs on 64-bit computers. The 64-bit version is useful for CIS/2 files greater than approximately $200 \mathrm{MB}$ and should be run on a computer with more than $2 \mathrm{~GB}$ of memory. The 64-bit command-line version has the same functionality as the other command-line version except that IFC files cannot be generated.

Either command-line version can be run from a Windows command prompt by going to the Windows start menu, selecting Run, and entering "cmd". This will open up the command prompt window. To run either version, change to the directory where the programs are located and enter either ctv or ctv- 64 and the name of the CIS/2 file to translate. Entering the name of the program without a CIS/ 2 file will display all of the command-line options as partially shown in Figure 62.

To facilitate running the command-line version, the PATH environment variable can be set to include the directory where the command-line executables are located. A batch file can also be created to run the command-line executables. If the CIS/2 file is not located in the same directory as the command-line executable, then the CIS/2 file name should also include the directory pathname for the file.

Most options that are available in the GUI version of SteelVis are also available in the command-line version. However, some options in the command-line version are combinations of individual options in the GUI version. For example, the command-line option "bhw" turns on bolts, holes, and welds.

As an example, the following command: ctv file.stp sum pop1 bhw web will translate the CIS/2 file with text popups (section 5.3.1 above), the summary report (section 5.1 above), bolts, holes, and welds (section 6.1.2 above) and the resulting VRML model will be displayed in the default web browser.

When the command-line version is run, feedback is provided that is similar to what is displayed in the Status tab (section 3.3 above) as shown in Figure 63.

The command-line version can also be embedded or accessed by other programs. For example, the command-line version of SteelVis has been embedded in several commercial software products including, FabSuite [20], StruWalker Plus [21], and ConstructSim to facilitate importing and visualizing CIS/2 files.

\section{Web Services Version}

The online web services version of SteelVis can be accessed at http://ciks.cbt.nist.gov/cgi-bin/ctv/ctv.cgi or through the Websites menu of the GUI version [22]. The web services version can be run on a computer with any operating system or web browser. Only a limited set of display options are available in the web services version as shown in Figure 64. IFC files cannot be generated from the web services version.

To run the web services version, use the Browse button to select a CIS/2 file on the computer. Then select the appropriate display options. Finally, use the Translate to VRML button to generate the VRML file. When the CIS/2 file is translated, it is uploaded to a server at NIST where it is processed by SteelVis and the resulting VRML file is sent back to the web browser where it can be displayed as either a text file or in the web browser VRML plugin. 


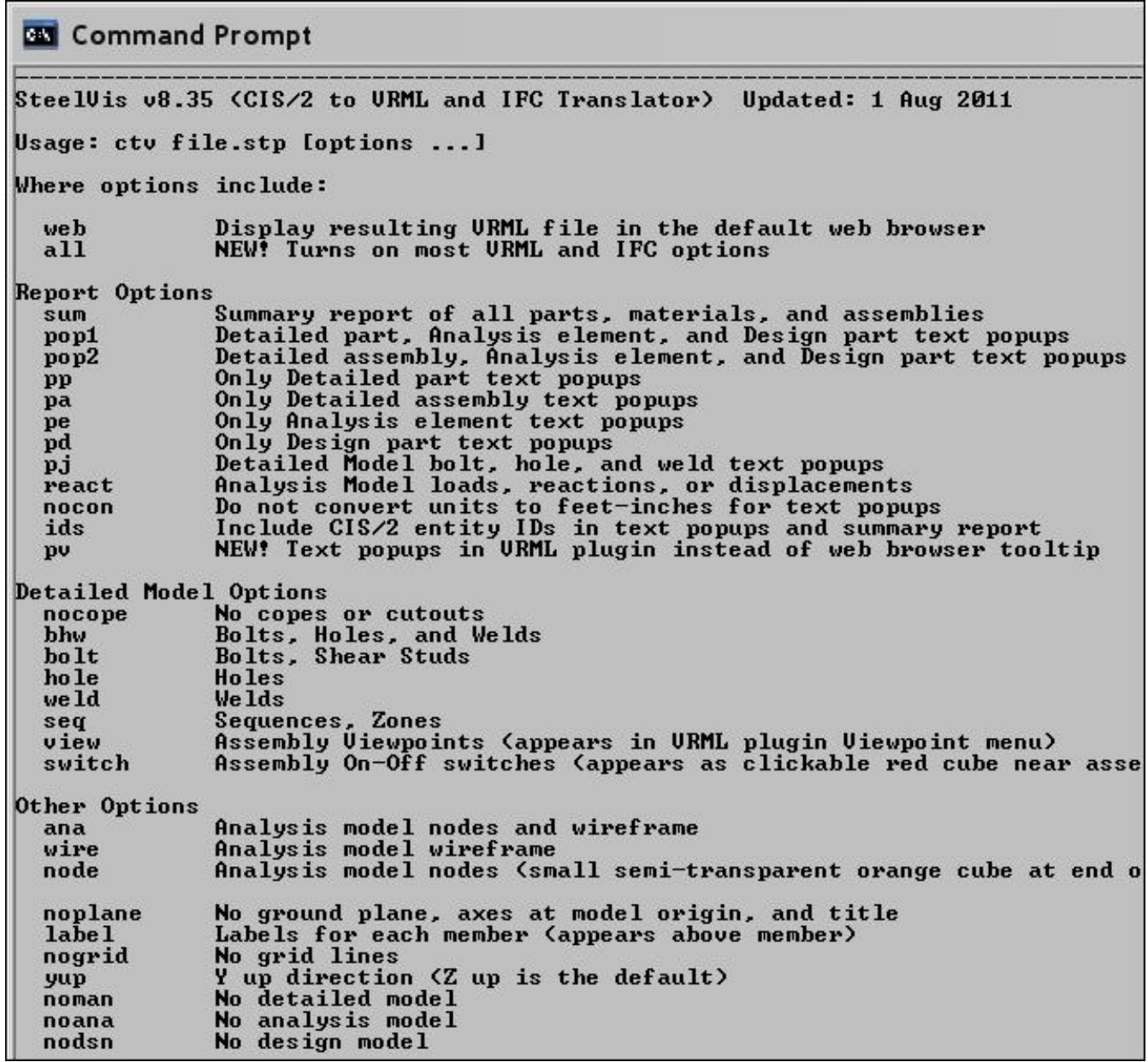

Figure 62: Command-line version options (partial list) 


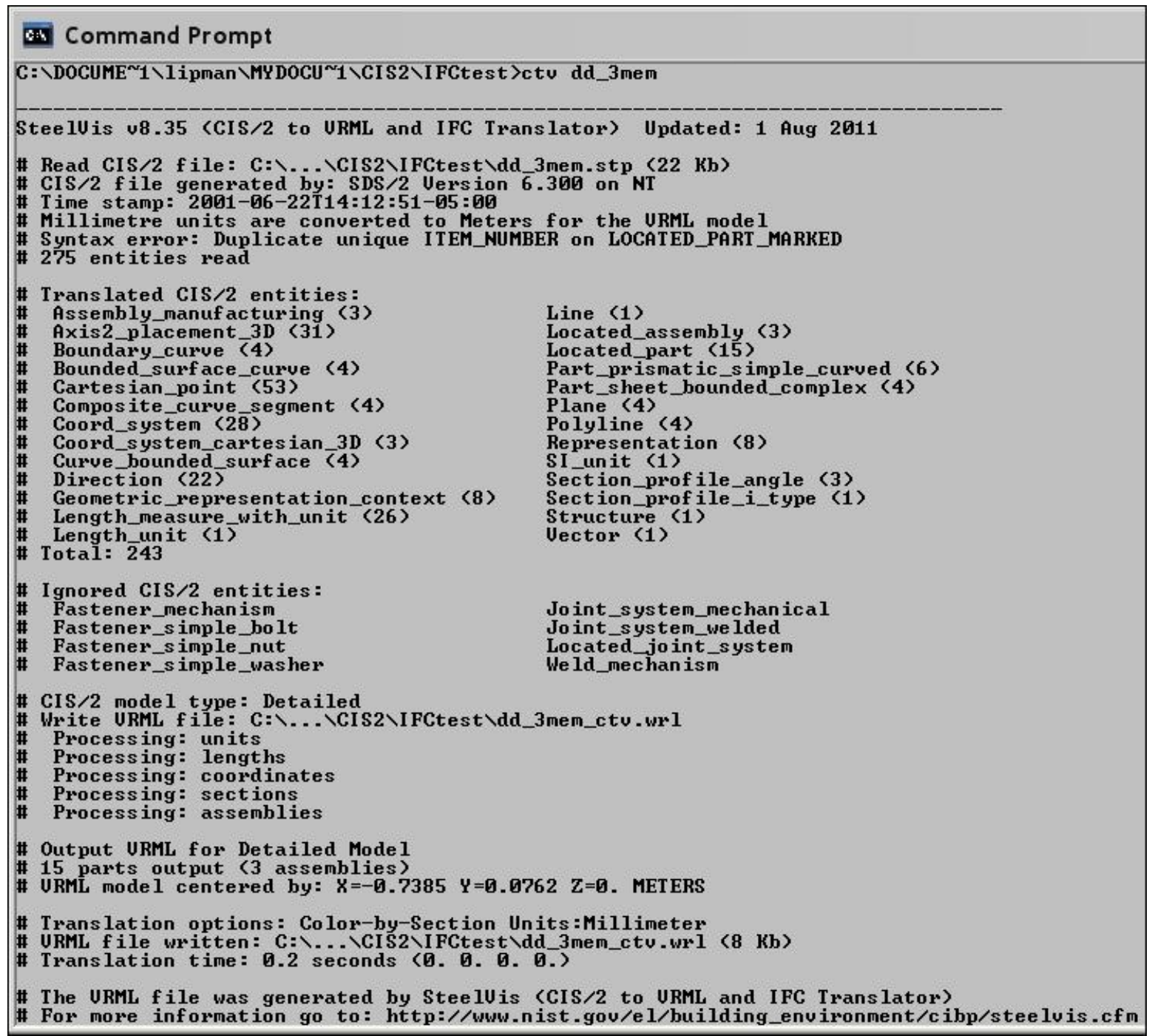

Figure 63: Feedback when running the command-line version 


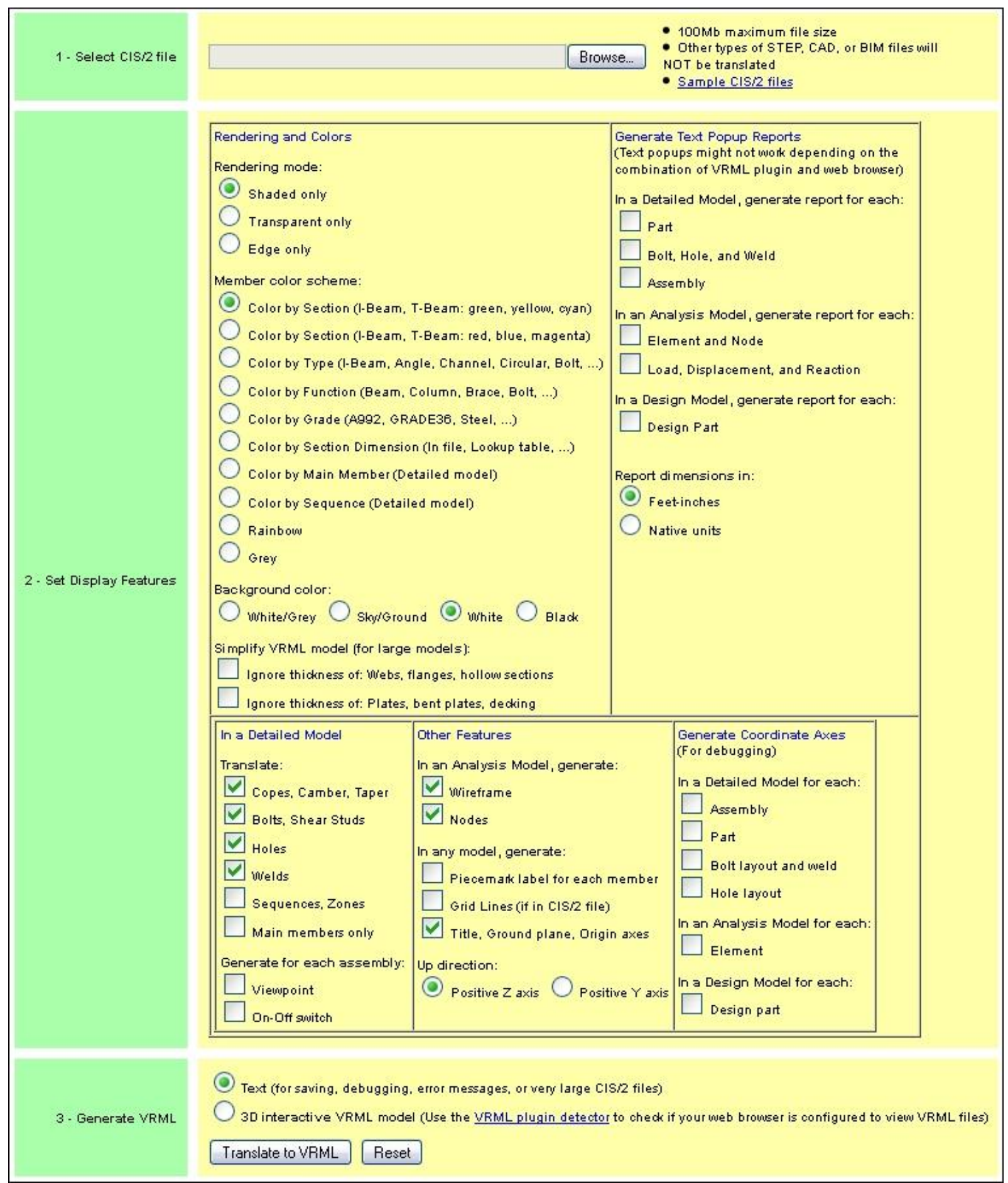

\section{Figure 64: SteelVis web services version}




\section{References}

(All websites were successfully accessed in January 2012).

[1] Crowley, A., Watson, A., "CIMsteel Integration Standards Release 2", SCI-P-268, The Steel Construction Institute, Berkshire, England, (2000), http://www.aisc.org/store/p-2021-cimsteelintegration-standards-release-2-complete-set.aspx.

[2] Eastman, C., Wang, F., You S.-J., and Yang, D., "Deployment of an AEC industry sector product model", Computer-Aided Design, Vol. 37-12, October 2005, 1214-1228, http://dx.doi.org/10.1016/j.cad.2004.11.007.

[3] A. Ames, D. Nadeau, and J. Moreland,VRML 2.0 Sourcebook, John Wiley \& Sons, Inc., New York (1997).

[4] VRML97 and Related Specifications, Web3D Consortium, http://www.web3d.org/x3d/specifications/vrml/.

[5] IFC Specifications, http://buildingsmart-tech.org/specifications/ifc-overview/ifc-overview-summary.

[6] buildingSMART, http://buildingsmart.com/.

[7] Harmonization for CIS/2 and IFC, http://tempest.arch.gatech.edu/ aisc/cisifc/.

[8] X3D, H-Anim, and VRML97 Specifications, Web3D Consortium, http://www.web3d.org/x3d/specifications/.

[9] X3DOM, http://www.X3dom.org/.

[10] IFC Quick Browser, http://www.team-solutions.de/?page id=19.

| [11] IFCsvr, http://tech.groups.yahoo.com/group/ifcsvr-users/.

[12] Lipman, R.R., "Developing Coverage Analysis for IFC Files", Proceedings of the CIB W78 Conference, Cairo, Egypt, November 2010, http://www.nist.gov/manuscript-publicationsearch.cfm?pub_id=906055.

[13] IFC File Analyzer, http://www.nist.gov/el/msid/infotest/ifc-file-analyzer.cfm.

[14] Express Engine, http://exp-engine.sourceforge.net/.

[15] NIST Expresso, http://www.nist.gov/el/msid/scl.cfm.

[16] Lipman, R.R., "Mapping Between the CIMsteel Integration Standards and Industry Foundation Classes Product Data Models for Structural Steel", Proceedings of the International Conference on Computing in Civil and Building Engineering, Montreal, Canada, June 2006, http://www.nist.gov/manuscript-publication-search.cfm?pub id=100936.

[17] Lipman, R.R., "Details of the Mapping Between the CIS/2 and IFC Product Data Models for Structural Steel", Journal of Information Technology in Construction, Vol 14, 1-50, (2009), http://www.nist.gov/manuscript-publication-search.cfm?pub_id=861673.

[18] IFC Coordination View Version 2.0 Summary, http://buildingsmart-tech.org/specifications/ifc-viewdefinition/coordination-view-v2.0/summary.

[19] IFC2x3 Final Documentation, http://buildingsmart-tech.org/ifc/IFC2x3/TC1/html/index.htm.

[20] FabSuite, http://www.youtube.com/user/FabSuite\#p/u/3/I659bZsXdHA.

[21] StruWalker Plus, http://www.youtube.com/user/AceCadSoftware\#p/u/10/EEtDTUV19PA.

[22] Lipman, R.R., Reed, K.A., "Visualization of Structural Steel Product Models", Journal of Information Technology in Construction, Vol 8, 51-64, (2003), http://www.nist.gov/manuscriptpublication-search.cfm?pub_id=860885. 